\title{
DOCUMENTARY APPENDIX
}

\author{
Compiled by Debora Mantovani
}

The documentary appendix presented in the following pages is intended to provide the reader with a general picture of the demographic, economic, and social situation that formed the background to the events of 2004 discussed earlier in this volume.

This year's appendix is divided into three sections. The first consists of tables A1 to A14, which provide data concerning resident population by sex and age group (table A1); population changes-live births, deaths, marriages (table A2); and foreign resident population, foreign resident population as a proportion of the total population, and residence permits issued by region and by purpose for which issued (tables A3 and A4). In addition, some important indicators of the state of the economy and of public finances are provided. These include gross domestic product, the size of the public debt, and public-sector borrowing (tables A5 and A6). Tables A7, A8, and A9 are devoted to a comparison of the situation in Italy with that of the other countries of the European Union and take account, wherever possible, of the changes that have come about as a result of enlargement and the entry of the 10 new member countries. The information provided concerns natural population movements and migration, a number of education indicators, and rates of unemployment by sex. Tables A10, A11, and A12 provide the principal information concerning the Italian labor force: resident population by employment status, sector of activity, and employment category (table A10); unemployment rates by geographical area, age group, and sex (table A11); unemployment rates by geographical area, age group, and educational qualifications (table A12). Table A13 is also concerned with educational qualifications and 
provides data, for 1991, 2001, and 2003, showing the percentage distribution of the resident population by educational qualification, age group, and sex. Finally, table A14 shows trends in reported crime for the years 1991, 2001, 2002, and 2003.

The second section provides data concerning the results of the numerous elections held in 2004. The provincial and local elections that took place in June involved voters resident in 63 provinces and 32 provincial capitals. In addition, in Sardinia, an election for the regional council took place. June 2004 also saw the European Parliament elections, while in October the Chamber of Deputies by-elections were held. Finally, on 21 March there was a consultative referendum on the creation of the autonomous province of Alto Friuli. Tables B1a, B1b, $\mathrm{B} 2$, and $\mathrm{B} 3$ provide data showing, for the municipal elections held in each of the provincial capitals, turnout, the distribution of the vote among party lists, and the distribution of the vote among mayoral candidates. Tables $\mathrm{B} 4 \mathrm{a}, \mathrm{B} 4 \mathrm{~b}, \mathrm{~B} 5$, and $\mathrm{B} 6$ provide the same information for the provincial elections. Tables B7a and B7b provide data for the European Parliament elections showing the turnout and the raw and percentage distributions of the vote among the various party lists. Data concerning turnout at the Sardinian regional election, together with the distribution of the vote between the regional and district lists and the presidential candidates, are shown in tables B8 to B11. Table B12 provides a summary of the results of the Alto Friuli referendum. Finally, table B13 provides information concerning the results of the Chamber of Deputies by-elections.

In the last section, table $\mathrm{C} 1$ provides membership figures for the principal Italian parties from 1992 to 2004 . It should be noted that the data reported in the table are based on figures supplied by the parties themselves. 


\section{A: BACKGROUND DATA}

TABLE A1. Resident Population by Age Group and Sex, on 1 January (percentages)

\begin{tabular}{lccccccc}
\hline Age & $\mathbf{0 - 1 4}$ & $\mathbf{1 5 - 2 4}$ & $\mathbf{2 5 - 4 4}$ & $\mathbf{4 5 - 6 4}$ & $\mathbf{6 5 +}$ & Total & $\mathbf{( N )}$ \\
\hline $\begin{array}{l}\text { Males and } \\
\text { Females }\end{array}$ & & & & & & & \\
$\quad 1981$ & 21.7 & 15.4 & 26.9 & 22.5 & 13.5 & 100 & $(57,140,355)$ \\
1991 & 16.3 & 15.8 & 29.1 & 24.0 & 14.8 & 100 & $(57,746,163)$ \\
$2001^{a}$ & 14.2 & 11.2 & 30.7 & 25.2 & 18.7 & 100 & $(56,995,744)$ \\
2003 & 14.2 & 10.8 & 30.7 & 25.3 & 19.0 & 100 & $(57,321,070)$
\end{tabular}

Males

$\begin{array}{llllllll}1981 & 22.8 & 16.0 & 27.7 & 22.0 & 11.5 & 100 & (27,900,645) \\ 1991 & 17.2 & 16.6 & 30.1 & 23.8 & 12.3 & 100 & (28,072,498) \\ 2001^{a} & 15.1 & 11.8 & 31.7 & 25.5 & 15.9 & 100 & (27,586,982) \\ 2003 & 15.1 & 11.4 & 31.9 & 25.5 & 16.2 & 100 & (27,766,223)\end{array}$

Females

$\begin{array}{llllllll}1981 & 20.7 & 14.7 & 26.2 & 22.9 & 15.5 & 100 & (29,239,710) \\ 1991 & 15.4 & 15.0 & 28.3 & 24.1 & 17.2 & 100 & (29,673,665) \\ 2001^{a} & 13.4 & 10.6 & 29.7 & 25.0 & 21.3 & 100 & (29,408,762) \\ 2003 & 13.4 & 10.2 & 29.6 & 25.0 & 21.7 & 100 & (29,554,847)\end{array}$

${ }^{a}$ Figures refer to 21 October 2001; data drawn from the 14th general census of the population.

Source: ISTAT, Popolazione e movimento anagrafico dei comuni (Rome, 2001); our elaboration based on data provided by the ISTAT Web site (http://dawinci .istat.it), relating to the 14th general census of the population. 
TABLE A2. Live Births, Deaths, and Marriages of the Resident Population

\begin{tabular}{|c|c|c|c|c|}
\hline Year & Total & \multicolumn{2}{|l|}{$\begin{array}{c}\text { Index } \\
(1981=100)\end{array}$} & $\begin{array}{c}\% \text { Natural } \\
\text { (of total born alive) }\end{array}$ \\
\hline 1981 & 621,805 & \multicolumn{2}{|l|}{100.0} & 4.2 \\
\hline 1991 & 562,787 & \multicolumn{2}{|l|}{90.5} & 6.7 \\
\hline $2001^{a}$ & 531,880 & \multicolumn{2}{|l|}{85.5} & 11.1 \\
\hline $2002^{a}$ & 535,538 & \multicolumn{2}{|l|}{86.1} & 12.3 \\
\hline $2003^{b}$ & 542,629 & \multicolumn{2}{|l|}{87.3} & 13.8 \\
\hline \multicolumn{5}{|c|}{ DEATHS } \\
\hline Year & Total & \multicolumn{2}{|l|}{$\begin{array}{c}\text { Index } \\
(1981=100)\end{array}$} & $\begin{array}{c}\text { Natural Balance } \\
\text { (live births-deaths) }\end{array}$ \\
\hline 1981 & 542,204 & \multicolumn{2}{|l|}{100.0} & $+79,601$ \\
\hline 1991 & 553,833 & \multicolumn{2}{|l|}{102.1} & $+8,954$ \\
\hline $2001^{a}$ & 555,247 & \multicolumn{2}{|l|}{102.4} & $-23,367$ \\
\hline $2002^{a}$ & 558,270 & \multicolumn{2}{|l|}{103.0} & $-22,732$ \\
\hline $2003^{b}$ & 594,118 & \multicolumn{2}{|l|}{109.6} & $-51,489$ \\
\hline \multicolumn{5}{|c|}{ MARRIAGES } \\
\hline Year & Total & $\begin{array}{c}\text { Index } \\
(1981=100)\end{array}$ & Religious & $\begin{array}{c}\% \text { Religious } \\
\text { (of total marriages) }\end{array}$ \\
\hline 1981 & 313,736 & 100.0 & 272,326 & 86.8 \\
\hline 1991 & 312,061 & 99.5 & 257,555 & 82.5 \\
\hline $2001^{a}$ & 260,904 & 83.2 & 190,888 & 73.2 \\
\hline $2002^{a}$ & 265,635 & 84.7 & 190,879 & 71.9 \\
\hline $2003^{b}$ & 258,580 & 82.4 & 184,790 & 71.5 \\
\hline
\end{tabular}

${ }^{a}$ Dati provvisori secondo gli atti dello stato civile. The data for live births refer to the regions in which the births were registered.

${ }^{b}$ Estimated figures.

Source: ISTAT, Annuario statistico italiano 2004 (Rome, 2004). 
TABLE A3. Total Resident and Foreign Population as of the 2001 Census by Region

\begin{tabular}{lrcc}
\hline Region & $\begin{array}{c}\text { Resident } \\
\text { Population }\end{array}$ & $\begin{array}{c}\text { Foreign } \\
\text { Population }\end{array}$ & $\begin{array}{c}\text { \% Foreign (of total } \\
\text { resident population) }\end{array}$ \\
\hline Valle d'Aosta & 119,548 & 2,630 & 2.2 \\
Piedmont & $4,214,677$ & 110,402 & 2.6 \\
Lombardy & $9,032,554$ & 319,564 & 3.5 \\
Trentino-Alto Adige & 940,016 & 30,326 & 3.2 \\
Veneto & $4,527,694$ & 153,074 & 3.4 \\
Friuli-Venezia Giulia & $1,183,764$ & 38,122 & 3.2 \\
Liguria & $1,571,783$ & 35,950 & 2.3 \\
Emilia-Romagna & $3,983,346$ & 135,453 & 3.4 \\
Tuscany & $3,497,806$ & 108,702 & 3.1 \\
Umbria & 825,826 & 27,266 & 3.3 \\
Marches & $1,470,581$ & 45,668 & 3.1 \\
Lazio & $5,112,413$ & 151,567 & 3.0 \\
Abruzzo & $1,262,392$ & 21,399 & 1.7 \\
Molise & 320,601 & 2,588 & 0.8 \\
Campania & $5,701,931$ & 40,430 & 0.7 \\
Apuglia & $4,020,707$ & 30,161 & 0.8 \\
Basilicata & 597,768 & 3,416 & 0.6 \\
Calabria & $2,011,466$ & 18,017 & 0.9 \\
Sicily & $4,968,991$ & 49,399 & 1.0 \\
Sardinia & $1,631,880$ & 10,755 & 0.7 \\
Italy & $56,995,744$ & $1,334,889$ & 2.3 \\
\hline & & & \\
\hline
\end{tabular}

Source: ISTAT, 2001 census. 


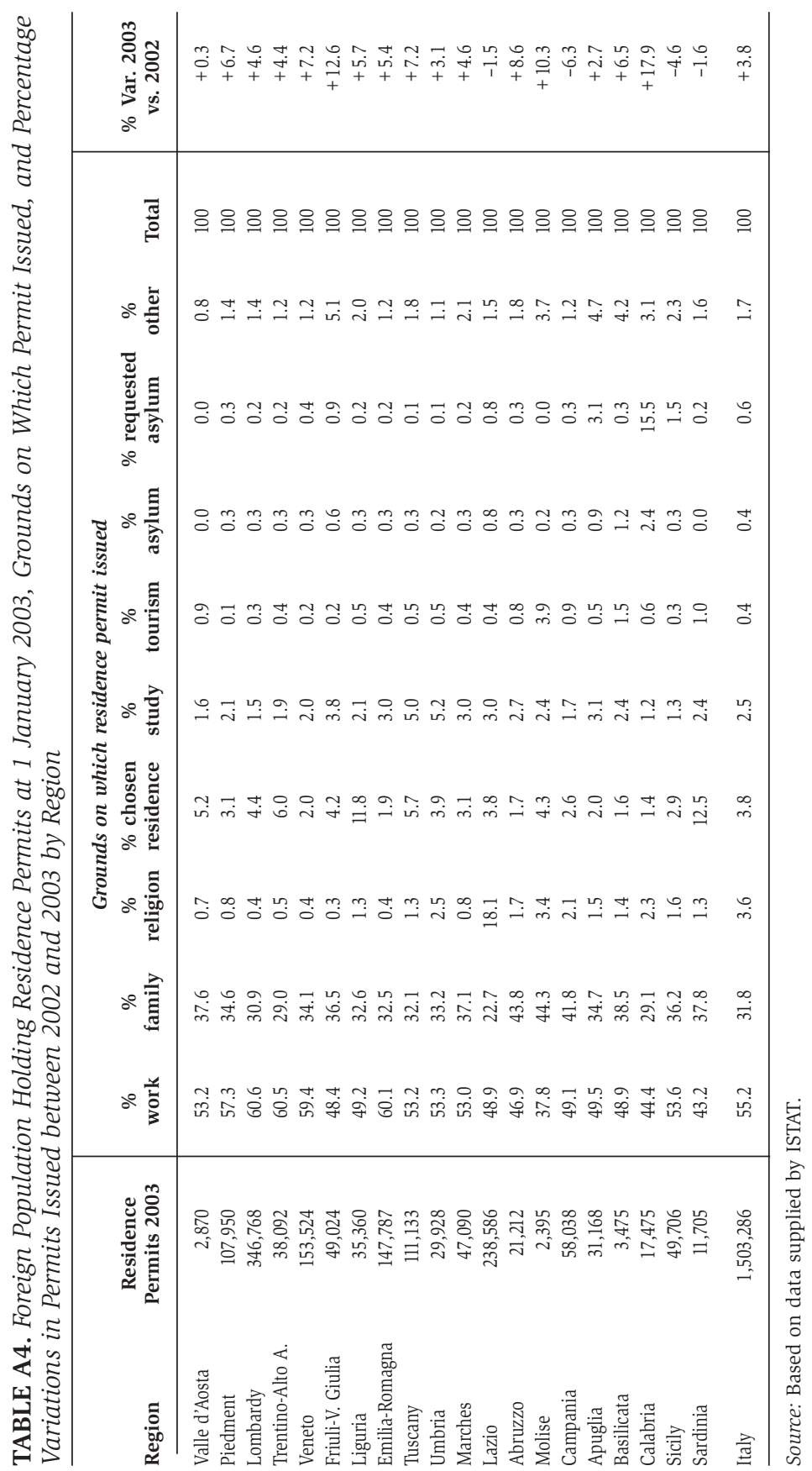


TABLE A5. Gross Domestic Product (at market prices) and Consumer Price Index (figures given in millions of euros)

\begin{tabular}{l|cccc|c}
\hline \multicolumn{5}{c}{ Gross DoMESTIC Product } \\
\hline & $\begin{array}{c}\text { Value at } \\
\text { current } \\
\text { prices }\end{array}$ & $\begin{array}{c}\text { \% Variation } \\
\text { on previous } \\
\text { Year }\end{array}$ & $\begin{array}{c}\text { Value at } \\
\mathbf{1 9 9 5} \\
\text { prices }\end{array}$ & $\begin{array}{c}\text { \% Variation } \\
\text { on previous } \\
\text { year }\end{array}$ & $\begin{array}{c}\text { \% Change } \\
\text { of price index } \\
\text { on prev. } \\
\text { year }\end{array}$ \\
\hline 1992 & 783,774 & +5.3 & 885,284 & +0.8 & +5.2 \\
1993 & 807,362 & +3.0 & 877,460 & -0.9 & +4.5 \\
1994 & 853,911 & +5.8 & 896,830 & +2.2 & +4.0 \\
1995 & 923,052 & +8.1 & 923,052 & +2.9 & +5.2 \\
1996 & 982,443 & +6.4 & 933,142 & +1.1 & +4.0 \\
1997 & $1,026,285$ & +4.5 & 952,050 & +2.0 & +2.0 \\
1998 & $1,073,019$ & +4.6 & 969,130 & +1.8 & +2.0 \\
1999 & $1,107,994$ & +3.3 & 985,253 & +1.7 & +1.7 \\
2000 & $1,166,548$ & +5.3 & $1,015,077$ & +3.0 & +2.5 \\
2001 & $1,218,535$ & +4.5 & $1,032,985$ & +1.8 & +2.7 \\
2002 & $1,260,428$ & +3.4 & $1,036,701$ & +0.4 & +2.5 \\
2003 & $1,300,926$ & +3.2 & $1,039,367$ & +0.3 & +2.7 \\
\hline
\end{tabular}

Source: ISTAT, Annuario statistico italiano 2004 (Rome, 2004).

TABLE A6. Public Debt and Public Sector Deficit (figures given in millions of euros)

\begin{tabular}{lccc|ccc}
\hline \multicolumn{3}{c|}{ PUBLIC DEBT } & \multicolumn{3}{c}{ PUBLIC SECTOR DEFICIT } \\
\hline & $\begin{array}{c}\text { \% Var. } \\
\text { Absolute } \\
\text { value }\end{array}$ & year & $\begin{array}{c}\text { \% of } \\
\text { GDP }\end{array}$ & $\begin{array}{c}\text { Absolute } \\
\text { value }\end{array}$ & $\begin{array}{c}\text { \% Var. } \\
\text { on prev. } \\
\text { year }\end{array}$ & $\begin{array}{c}\text { \% of } \\
\text { GDP }\end{array}$ \\
\hline 1992 & 843,891 & +12.8 & 107.7 & 74,191 & -0.3 & 9.5 \\
1993 & 954,691 & +13.1 & 118.2 & 75,877 & +2.3 & 9.4 \\
1994 & $1,061,802$ & +11.2 & 124.3 & 79,370 & +4.6 & 9.3 \\
1995 & $1,142,788$ & +7.6 & 123.8 & 70,081 & -11.7 & 7.6 \\
1996 & $1,205,392$ & +5.5 & 122.7 & 69,746 & -0.5 & 7.1 \\
1997 & $1,233,683$ & +2.3 & 120.2 & 27,723 & -60.3 & 2.7 \\
1998 & $1,248,469$ & +1.2 & 116.4 & 30,198 & +8.9 & 2.8 \\
1999 & $1,273,243$ & +2.0 & 114.9 & 19,125 & -36.7 & 1.7 \\
2000 & $1,297,100$ & +1.9 & 111.2 & $21,359^{a}$ & +11.7 & 1.8 \\
2001 & $1,347,805$ & +3.9 & 110.6 & 32,262 & +51.0 & 2.6 \\
2002 & $1,360,253$ & +0.9 & 111.6 & 28,403 & -12.0 & 2.3 \\
2003 & $1,381,394$ & +1.6 & 109.6 & 31,832 & +12.1 & 2.4 \\
\hline
\end{tabular}

${ }^{a}$ The figure for 2000 does not include the proceeds from UMTS licenses (€13,815 milion; in national accounts this revenue is registered against costs.

Source: Bank of Italy, Relazione annuale sul 2003 (Rome, 2004). 
TABLE A7. Population Density and Changes in EU Countries in 2002 (population changes in thousands)

\begin{tabular}{|c|c|c|c|c|c|c|c|}
\hline Country & $\begin{array}{c}\text { Density } \\
\text { (inhabi- } \\
\text { tants } \\
\text { per } \mathrm{km}^{2} \text { ) }\end{array}$ & Births & Deaths & $\begin{array}{l}\text { Natural } \\
\text { balance }\end{array}$ & $\begin{array}{r}\text { Migrator } \\
\text { balance }^{d}\end{array}$ & $\begin{array}{cc}\text { ry } & \begin{array}{c}\text { Total } \\
\mathrm{e}^{a} \\
\text { balance }\end{array}\end{array}$ & $\begin{array}{l}\text { Population } \\
\text { at year } \\
\text { end }\end{array}$ \\
\hline Austria & 96.0 & 78.4 & 76.1 & +2.3 & +26.1 & +28.4 & $8,067.3$ \\
\hline Belgium & 338.6 & 111.2 & 105.6 & +5.6 & +40.5 & +46.1 & $10,335.8$ \\
\hline Denmark & 124.8 & 64.1 & 58.6 & +5.5 & +9.6 & +15.2 & $5,383.5$ \\
\hline Finland & 15.4 & 55.6 & 49.4 & +6.1 & +5.3 & +11.4 & $5,206.3$ \\
\hline France & 107.9 & 761.6 & 539.7 & +221.9 & +66.1 & +288.0 & $59,630.1^{b}$ \\
\hline Germany & 231.0 & 719.3 & 841.7 & -122.4 & +218.8 & +96.4 & $82,536.7^{c}$ \\
\hline Greece & 83.4 & $102.5^{c}$ & $104.2^{c}$ & $-1.7^{c}$ & $+32.1^{c}$ & $+30.4^{c}$ & $11,018.4^{c}$ \\
\hline Ireland & 55.9 & 60.5 & 29.4 & +31.1 & +32.5 & +63.7 & $3,963.6$ \\
\hline Italy & 189.7 & 538.2 & 557.4 & -19.2 & +346.5 & +327.3 & $53,321.0^{c}$ \\
\hline Luxembourg & 172.5 & 5.3 & 3.7 & +1.6 & +2.6 & +4.3 & 448.3 \\
\hline Netherlands & 388.9 & 202.1 & 142.4 & +59.7 & +27.6 & +87.3 & $16,192.6$ \\
\hline Portugal & 112.2 & 114.4 & 106.3 & +8.1 & +70.0 & +78.1 & $10,407.5$ \\
\hline Spain & 81.6 & 411.5 & 365.8 & +45.8 & +654.3 & +700.1 & $41,550.6^{c}$ \\
\hline Sweden & 19.8 & 95.8 & 94.6 & +1.2 & +30.5 & +31.7 & $8,940.8$ \\
\hline UK & 245.0 & 668.8 & 606.3 & +62.5 & +126.5 & +189.0 & $59,328.9^{c}$ \\
\hline EU (15 countries) & 117.1 & $3,989.3$ & 3.681 .1 & +308.2 & $+1,689.0$ & +1.997 .2 & $380,351.4^{c}$ \\
\hline Cyprus & 76.8 & 8.9 & 5.8 & +3.1 & +6.5 & +9.6 & 715.1 \\
\hline Czech Rep. & 129.4 & 92.8 & 108.2 & -15.5 & +12.4 & -3.1 & $10,203.3$ \\
\hline Estonia & 30.0 & 13.0 & 18.4 & -5.4 & +0.2 & -5.2 & $1,356.0$ \\
\hline Hungary & 109.2 & 96.8 & 132.8 & -36.0 & +3.5 & -32.5 & $10,142.4$ \\
\hline Latvia & 36.2 & 20.0 & 32.5 & -12.5 & -1.8 & -14.3 & $2,331.5$ \\
\hline Lithuania & 53.1 & 30.0 & 41.1 & -11.1 & -2.0 & -13.0 & $3,462.6$ \\
\hline Malta & 1.253 .0 & 3.8 & 3.0 & +0.8 & +1.9 & +2.7 & 397.3 \\
\hline Poland & 122.9 & 353.8 & 359.5 & -5.7 & -12.6 & -18.4 & $38,215.5^{d}$ \\
\hline Slovakia & 109.7 & 50.8 & 51.5 & -0.7 & +0.9 & +0.2 & $5,379.2$ \\
\hline Slovenia & 98.4 & 17.5 & 18.7 & -1.2 & +2.2 & +1.0 & $1,995.0$ \\
\hline EU (25 countries) & 114.0 & $4,676.8$ & 4.452 .7 & 224.1 & $1,700.2$ & 1.924 .3 & $454,552.3^{d}$ \\
\hline
\end{tabular}

${ }^{a}$ Includes post-census corrections or the regularization of the register regarding events other than births, deaths, or migration.

${ }^{b}$ Provisional figures.

cEstimate.

${ }^{d}$ Series suspended.

Source: ISTAT, Annuario statistico italiano 2004 (Rome, 2004). 
Table A8. Educational Indicators for Certain Countries (academic year 2000-2001)

\begin{tabular}{|c|c|c|c|c|c|c|}
\hline \multirow[b]{2}{*}{ Country } & \multicolumn{2}{|c|}{$\begin{array}{l}\text { Public spending on } \\
\text { education as } \% \text { of } \mathrm{GDP}^{a}\end{array}$} & \multirow[b]{2}{*}{$\begin{array}{l}\text { Schooling } \\
\text { expectation } \\
\text { (in years) }^{b}\end{array}$} & \multirow[b]{2}{*}{$\begin{array}{c}\text { School } \\
\text { enrollment } \\
\text { rate } 15-19 \\
\text { years of age }\end{array}$} & \multicolumn{2}{|c|}{ Rates of attainment ${ }^{d}$} \\
\hline & $\begin{array}{l}\text { Total } \\
\text { (including } \\
\text { indivisible } \\
\text { expenses) }\end{array}$ & $\begin{array}{l}\text { Higher } \\
\text { education } \\
\text { spending } \\
\text { only }\end{array}$ & & & $\begin{array}{l}\text { 2nd grade } \\
\text { secondary } \\
\text { education }\end{array}$ & $\begin{array}{c}\text { Higher } \\
\text { education }\end{array}$ \\
\hline \multicolumn{7}{|c|}{ European Union } \\
\hline Austria & 5.7 & 1.2 & 16.3 & 77 & - & 16.6 \\
\hline Belgium & 5.5 & 1.3 & 19.2 & 91 & 79 & - \\
\hline Denmark & 6.7 & 1.6 & 18.0 & 83 & 96 & 38.8 \\
\hline Finland & 5.6 & 1.7 & 19.2 & 85 & 91 & 40.7 \\
\hline France & 6.1 & 1.1 & 16.6 & 87 & 85 & 25.0 \\
\hline Germany & 5.3 & 1.0 & 17.3 & 89 & 92 & 19.0 \\
\hline Greece & 4.0 & 0.9 & 16.1 & 77 & - & - \\
\hline Ireland & 4.6 & 1.5 & 16.3 & 81 & 76 & 29.3 \\
\hline Italy & 4.9 & 0.8 & 16.1 & 71 & 79 & 18.1 \\
\hline Luxembourg & - & - & - & 78 & - & - \\
\hline Netherlands & 4.7 & 1.3 & 17.3 & 86 & - & - \\
\hline Portugal & 5.7 & 1.1 & 17.1 & 73 & - & - \\
\hline Spain & 4.9 & 1.2 & 17.3 & 80 & 67 & 32.1 \\
\hline Sweden & 6.5 & 1.7 & 20.0 & 86 & 71 & 29.6 \\
\hline UK & 5.3 & 1.0 & 18.9 & 75 & - & 37.4 \\
\hline \multicolumn{7}{|c|}{ Other OECD Countries } \\
\hline Australia & 6.0 & 1.6 & 20.6 & 81 & - & 42.0 \\
\hline Canada & 6.4 & 2.6 & 16.5 & 75 & - & - \\
\hline Japan & 4.6 & 1.1 & - & - & 93 & 32.8 \\
\hline United States & 7.0 & 2.7 & 17.1 & 77 & 72 & - \\
\hline
\end{tabular}

${ }^{a}$ The indicator expresses public expenditure on education as a percentage of GDP for all levels of education, considering direct public expenditure on schools and all government subsidies to families.

${ }^{b}$ The figures for schooling expectation show the expected mean number of years of schooling for a child of five years during the course of his or her lifetime. 'The school enrollment rate for 15- to 19 -year-olds is the number of 15 - to 19 year-olds enrolled at all levels of education expressed as a percentage of the total population in that age category.

${ }^{d}$ The rate of attainment is the number of students obtaining, at the first attempt, second grade secondary or higher education qualifications expressed as a percentage of the total population in the corresponding age category attempting to obtain the qualification.

Source: ISTAT, Annuario statistico italiano 2004 (Rome, 2004). 
TABLE A9. Rate of Unemployment by Sex in the 25 Countries of the European Union, for the Years 2002, 2003, and 2004 (among persons aged 15 and above)

\begin{tabular}{|c|c|c|c|c|c|c|c|c|c|}
\hline \multicolumn{10}{|c|}{ Rate of Unemployment ${ }^{a}$} \\
\hline & \multicolumn{3}{|c|}{ Males } & \multicolumn{3}{|c|}{ Females } & \multicolumn{3}{|c|}{ Total } \\
\hline & 2002 & 2003 & 2004 & 2002 & 2003 & 2004 & 2002 & 2003 & 2004 \\
\hline Austria & 3.9 & 3.9 & 3.9 & 4.4 & 4.7 & 5.2 & 4.2 & 4.3 & 4.5 \\
\hline Belgium & 6.7 & 7.6 & 7.0 & 8.2 & 8.4 & 8.8 & 7.3 & 8.0 & 7.8 \\
\hline Cyprus & 3.0 & 3.9 & 4.0 & 4.9 & 5.2 & 6.3 & 3.9 & 4.5 & 5.0 \\
\hline Czech Rep. & 5.9 & 6.2 & 7.1 & 9.0 & 9.9 & 9.8 & 7.3 & 7.8 & 8.3 \\
\hline Denmark & 4.4 & 5.3 & 5.2 & 4.7 & 5.9 & 5.7 & 4.6 & 5.6 & 5.4 \\
\hline Estonia & 10.1 & 10.5 & 10.3 & 8.9 & 9.9 & 8.1 & 9.5 & 10.2 & 9.2 \\
\hline Finland & 9.1 & 9.2 & 8.8 & 9.1 & 8.9 & 9.0 & 9.1 & 9.0 & 8.9 \\
\hline France & 7.9 & 8.6 & 8.7 & 10.0 & 10.5 & 10.6 & 8.9 & 9.5 & 9.6 \\
\hline Germany & 8.8 & 10.0 & 10.1 & 8.4 & 9.2 & 9.4 & 8.7 & 9.6 & 9.8 \\
\hline Greece & 6.8 & 6.2 & - & 15.6 & 15.0 & - & 10.3 & 9.7 & - \\
\hline Hungary & 6.0 & 6.0 & 5.8 & 5.1 & 5.5 & 6.0 & 5.6 & 5.8 & 5.9 \\
\hline Ireland & 4.6 & 4.9 & 4.9 & 4.0 & 4.2 & 3.9 & 4.3 & 4.6 & 4.5 \\
\hline Italy & 7.0 & 6.7 & - & 12.2 & 11.6 & - & 9.0 & 8.6 & - \\
\hline Latvia & 13.6 & 10.1 & 9.2 & 11.4 & 10.6 & 10.3 & 12.6 & 10.4 & 9.8 \\
\hline Lithuania & 13.6 & 12.3 & 10.3 & 13.4 & 13.1 & 11.3 & 13.5 & 12.7 & 10.8 \\
\hline Luxembourg & 2.1 & 3.0 & 3.4 & 3.8 & 4.6 & 5.3 & 2.8 & 3.7 & 4.2 \\
\hline Malta & 6.7 & 6.8 & 6.9 & 9.8 & 10.7 & 8.4 & 7.7 & 8.0 & 7.4 \\
\hline Netherlands & 2.5 & 3.6 & 4.3 & 3.0 & 4.0 & 5.0 & 2.7 & 3.8 & 4.6 \\
\hline Poland & 19.0 & 18.6 & 18.1 & 20.7 & 20.0 & 19.8 & 19.8 & 19.2 & 18.9 \\
\hline Portugal & 4.1 & 5.4 & 5.7 & 6.0 & 7.2 & 7.5 & 5.0 & 6.3 & 6.6 \\
\hline Slovakia & 18.6 & 17.2 & 17.0 & 18.9 & 17.8 & 19.3 & 18.7 & 17.5 & 18.0 \\
\hline Slovenia & 5.8 & 6.0 & 5.6 & 6.5 & 7.0 & 6.5 & 6.1 & 6.5 & 6.0 \\
\hline Spain & 8.0 & 8.2 & 8.0 & 16.4 & 15.9 & 14.8 & 11.3 & 11.3 & 10.8 \\
\hline Sweden & 5.3 & 6.0 & 6.6 & 4.6 & 5.2 & 6.1 & 4.9 & 5.6 & 6.3 \\
\hline UK & 5.6 & 5.5 & 5.1 & 4.5 & 4.3 & 4.2 & 5.1 & 4.9 & 4.7 \\
\hline EU ( 25 countries) & 8.1 & 8.3 & 8.3 & 9.9 & 10.0 & 10.0 & 8.1 & 9.1 & 9.0 \\
\hline EU (15 countries) & 7.0 & 7.4 & 7.3 & 8.7 & 9.0 & 9.0 & 7.7 & 8.1 & 8.0 \\
\hline
\end{tabular}

${ }^{a}$ The rate of unemployment is the number of persons unemployed expressed as a percentage of the total labor force. The labor force is the sum of the number of persons employed and the number unemployed. The unemployed comprise persons between the ages of 15 and 74 who (a) were without work during the week of observation; (b) were willing to undertake paid employment (as employees or on their own account) in the two weeks subsequent to the week of observation; (c) had taken specific steps to find paid employment (as employees or on their own account) in the four weeks prior to the week of observation (including the latter) or had found employment that was due to start some time within the subsequent three months.

Source: Own elaboration of data made available through the Eurostat Web site: http://epp.eurostat.cec.eu.int/portal/page?_pageid = 1133,1406352,1133_ $1406373 \& \_$dad $=$portal\&_schema $=$PORTAL . 
TABLE A10. Resident Population by Employment Status and Sex, and Employed Population by Sector of Activity, Employment Category, and Sex for the Years 2000, 2001, 2002, and 2003 (percentages)

\begin{tabular}{|c|c|c|c|c|c|c|c|c|}
\hline & \multicolumn{2}{|r|}{2000} & \multicolumn{2}{|c|}{2001} & \multicolumn{2}{|c|}{2002} & \multicolumn{2}{|c|}{2003} \\
\hline & M & $\mathrm{F}$ & M & $\mathrm{F}$ & M & $\mathrm{F}$ & M & $\mathrm{F}$ \\
\hline \multicolumn{9}{|c|}{ Resident population by employment status } \\
\hline Employed & 91.9 & 85.5 & 92.6 & 87.0 & 93.1 & 87.8 & 93.2 & 88.4 \\
\hline Unemployed & 3.6 & 4.1 & 3.4 & 3.7 & 3.2 & 3.6 & 3.1 & 3.5 \\
\hline In search of first job & 3.7 & 5.4 & 3.3 & 4.9 & 3.0 & 4.6 & 3.0 & 4.2 \\
\hline Others & 0.8 & 5.0 & 0.7 & 4.4 & 0.7 & 4.0 & 0.7 & 3.9 \\
\hline Total labor force & 100 & 100 & 100 & 100 & 100 & 100 & 100 & 100 \\
\hline ( $\mathrm{N}$ in thousands) & $(14,495)$ & $(9,080)$ & $(14,521)$ & $(9,261)$ & $(14,609)$ & $(9,383)$ & $(14,685)$ & $(9,465)$ \\
\hline \multicolumn{9}{|c|}{ Population employed in various sectors of activity } \\
\hline Agriculture & 5.8 & 4.5 & 5.7 & 4.5 & 5.5 & 4.2 & 5.4 & 3.9 \\
\hline Industry & 38.6 & 21.0 & 38.6 & 20.4 & 38.8 & 20.1 & 39.1 & 19.9 \\
\hline Other activities & 55.6 & 74.5 & 55.7 & 75.1 & 55.7 & 75.7 & 55.5 & 76.2 \\
\hline Total employed & 100 & 100 & 100 & 100 & 100 & 100 & 100 & 100 \\
\hline ( $\mathrm{N}$ in thousands) & $(13,316)$ & $(7,764)$ & $(13,455)$ & $(8,060)$ & $(13,593)$ & $(8,236)$ & $(13,690)$ & $(8,365)$ \\
\hline \multicolumn{9}{|c|}{ Employed population by employment category } \\
\hline Entrepreneurs & 3.1 & 1.4 & 3.2 & 1.4 & 3.6 & 1.6 & 3.7 & 1.7 \\
\hline Self-employed professionals & 5.7 & 3.2 & 6.0 & 3.3 & 5.9 & 3.4 & 5.9 & 3.4 \\
\hline Own-account workers & 18.8 & 10.3 & 18.2 & 9.8 & 17.7 & 9.4 & 17.5 & 9.3 \\
\hline Partners in cooperative & & & & & & & & \\
\hline production & 1.4 & 1.2 & 1.2 & 1.0 & 0.9 & 0.9 & 0.9 & 0.9 \\
\hline Assistants & 2.8 & 5.9 & 3.1 & 6.0 & 3.0 & 5.9 & 3.0 & 5.8 \\
\hline Senior managers & 2.1 & 0.9 & 2.0 & 0.8 & 1.9 & 0.9 & 1.9 & 0.9 \\
\hline Executives & 4.7 & 4.5 & 5.0 & 4.7 & 4.8 & 4.6 & 4.7 & 4.5 \\
\hline White-collar employees & 23.9 & 43.1 & 24.2 & 44.0 & 24.7 & 44.5 & 24.9 & 45.1 \\
\hline Blue-collar employees & 36.6 & 28.3 & 36.3 & 27.9 & 36.6 & 27.7 & 36.8 & 27.4 \\
\hline Apprentices & 0.8 & 0.9 & 0.8 & 0.8 & 0.8 & 0.9 & 0.7 & 0.8 \\
\hline Home workers & 0.1 & 0.3 & 0.0 & 0.3 & 0.0 & 0.2 & 0.0 & 0.2 \\
\hline Total employed & 100 & 100 & 100 & 100 & 100 & 100 & 100 & 100 \\
\hline ( $\mathrm{N}$ in thousands) & $(13,316)$ & $7,764)$ & $(13,455)$ & $(8,060)$ & $(13,593)$ & $(8,236)$ & $(13,690)$ & $(8,365)$ \\
\hline
\end{tabular}

Source: ISTAT, Annuario statistico italiano 2004 (Rome, 2004); ISTAT, Forze di lavoro, media 2003 (Rome, 2004). 
TABLE A11. Rates of Unemployment by Geographical Area, Age Group, and Sex, for 2003 Among Persons Aged 15 and Over (percentages)

\begin{tabular}{lrrrrr}
\hline Age group and sex & Northwest & Northeast & Center & South & Italy \\
\hline Males & 2.9 & 2.2 & 4.5 & 13.8 & 6.8 \\
$15-24$ & 11.6 & 7.6 & 19.3 & 43.1 & 24.2 \\
$25-34$ & 3.5 & 2.7 & 6.4 & 19.4 & 9.2 \\
$35-54$ & 1.5 & 1.1 & 2.2 & 7.3 & 3.5 \\
$55-64$ & 1.9 & 1.7 & 2.3 & 6.4 & 3.6 \\
65 and over & 0.5 & 1.1 & 0.6 & 3.9 & 1.6 \\
Females & & & & & \\
$15-24$ & 6.0 & 4.7 & 9.3 & 25.3 & 11.6 \\
$25-34$ & 16.1 & 10.3 & 26.1 & 58.3 & 30.9 \\
$35-54$ & 6.5 & 5.5 & 13.1 & 33.2 & 14.4 \\
$55-64$ & 4.1 & 3.5 & 5.7 & 15.6 & 7.3 \\
65 and over & 3.6 & 2.5 & 2.6 & 7.3 & 4.3 \\
Total & 2.9 & 4.8 & 3.3 & 8.8 & 4.9 \\
15-24 & & & & & \\
$25-34$ & 4.2 & 3.2 & 6.5 & 17.7 & 8.7 \\
35-54 & 13.6 & 8.8 & 22.4 & 49.1 & 27.1 \\
55-64 & 4.9 & 4.0 & 9.3 & 24.3 & 11.4 \\
65 and over & 2.6 & 2.1 & 3.7 & 10.0 & 5.0 \\
\hline
\end{tabular}

Source: ISTAT, Forze di lavoro, media 2003 (Rome, 2004). 
TABLE A12. Rates of Unemployment by Geographical Area, Age Group, and Educational Qualification, for the Year 2003 (percentages)

\begin{tabular}{lccccc}
\hline $\begin{array}{l}\text { Age group and educa- } \\
\text { tional qualification }\end{array}$ & Northwest & Northeast & Center & South & Italy \\
\hline 25-34 & 4.9 & 4.0 & 9.3 & 24.3 & 11.4 \\
$\begin{array}{l}\text { No qualification or primary } \\
\quad \text { school diploma }\end{array}$ & 6.8 & 7.0 & 9.0 & 31.4 & 19.1 \\
Middle school diploma & 5.6 & 4.1 & 10.0 & 24.0 & 12.2 \\
Professional qualification & 3.1 & 3.5 & 7.7 & 23.3 & 7.0 \\
High school diploma & 4.0 & 2.6 & 7.8 & 22.8 & 10.1 \\
University degree & 6.4 & 7.5 & 13.0 & 27.6 & 13.8 \\
& & & & & \\
35-64 & 2.5 & 2.1 & 3.5 & 9.5 & 4.8 \\
No qualification or primary & & & & & \\
$\quad$ school diploma & 4.1 & 3.1 & 5.1 & 15.5 & 8.4 \\
Middle school diploma & 3.3 & 2.6 & 4.6 & 12.2 & 6.2 \\
Professional qualification & 2.8 & 2.1 & 3.3 & 8.7 & 3.7 \\
High school diploma & 1.6 & 1.4 & 2.7 & 6.2 & 3.2 \\
University degree & 1.1 & 0.9 & 1.5 & 2.6 & 1.6 \\
& & & & & \\
25-64 & 3.3 & 2.7 & 5.2 & 14.1 & 6.9 \\
No qualification or primary & & & & & \\
$\quad$ school diploma & 4.3 & 3.4 & 5.4 & 17.6 & 9.5 \\
Middle school diploma & 4.0 & 3.1 & 6.2 & 16.0 & 8.0 \\
Professional qualification & 2.9 & 2.6 & 4.6 & 13.4 & 4.8 \\
High school diploma & 2.5 & 1.9 & 4.6 & 12.4 & 5.9 \\
University degree & 3.0 & 3.3 & 5.1 & 10.1 & 5.7 \\
\hline So & & & & &
\end{tabular}

Source: ISTAT, Forze di lavoro, media 2003 (Rome, 2004). 


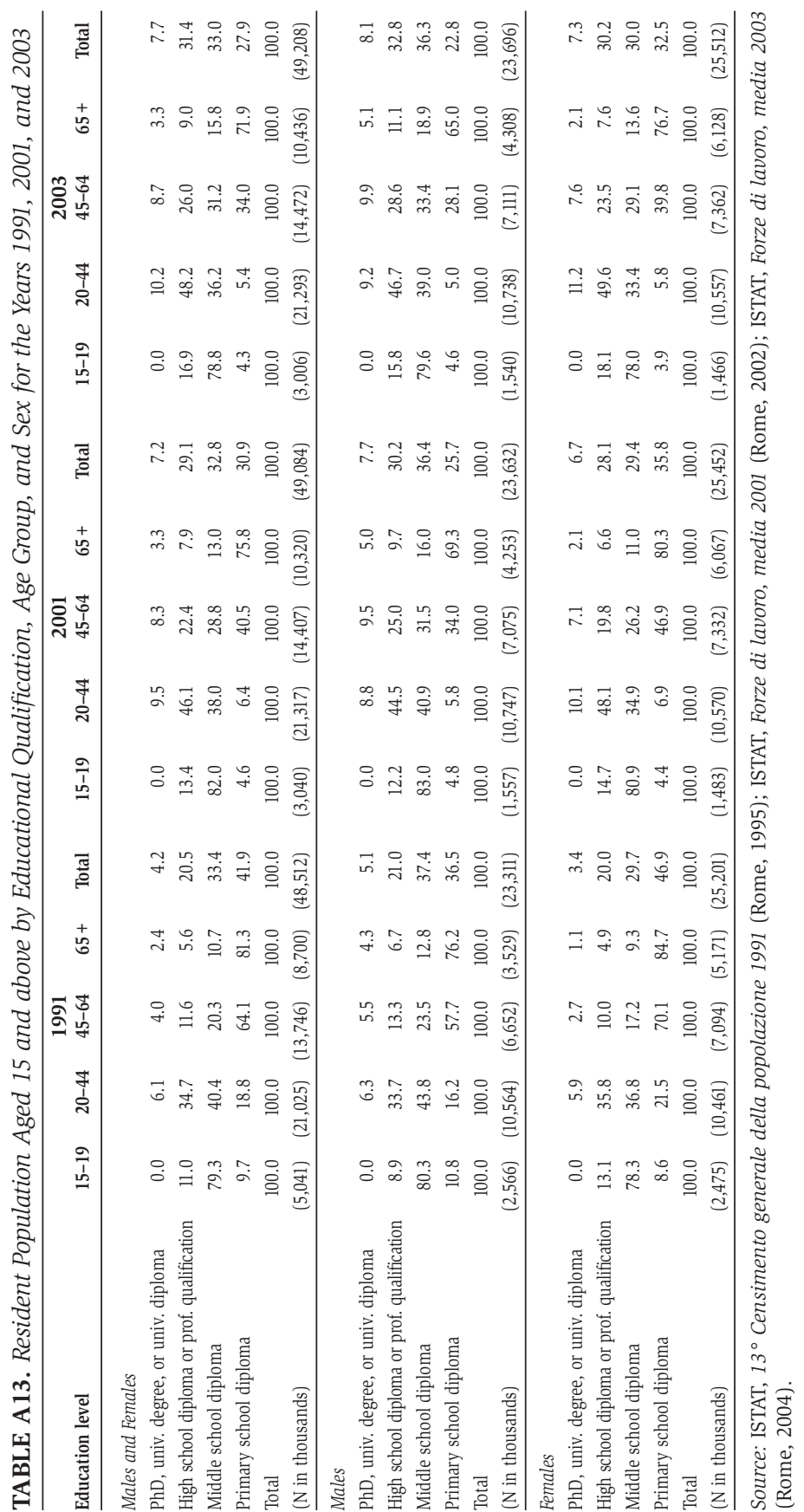




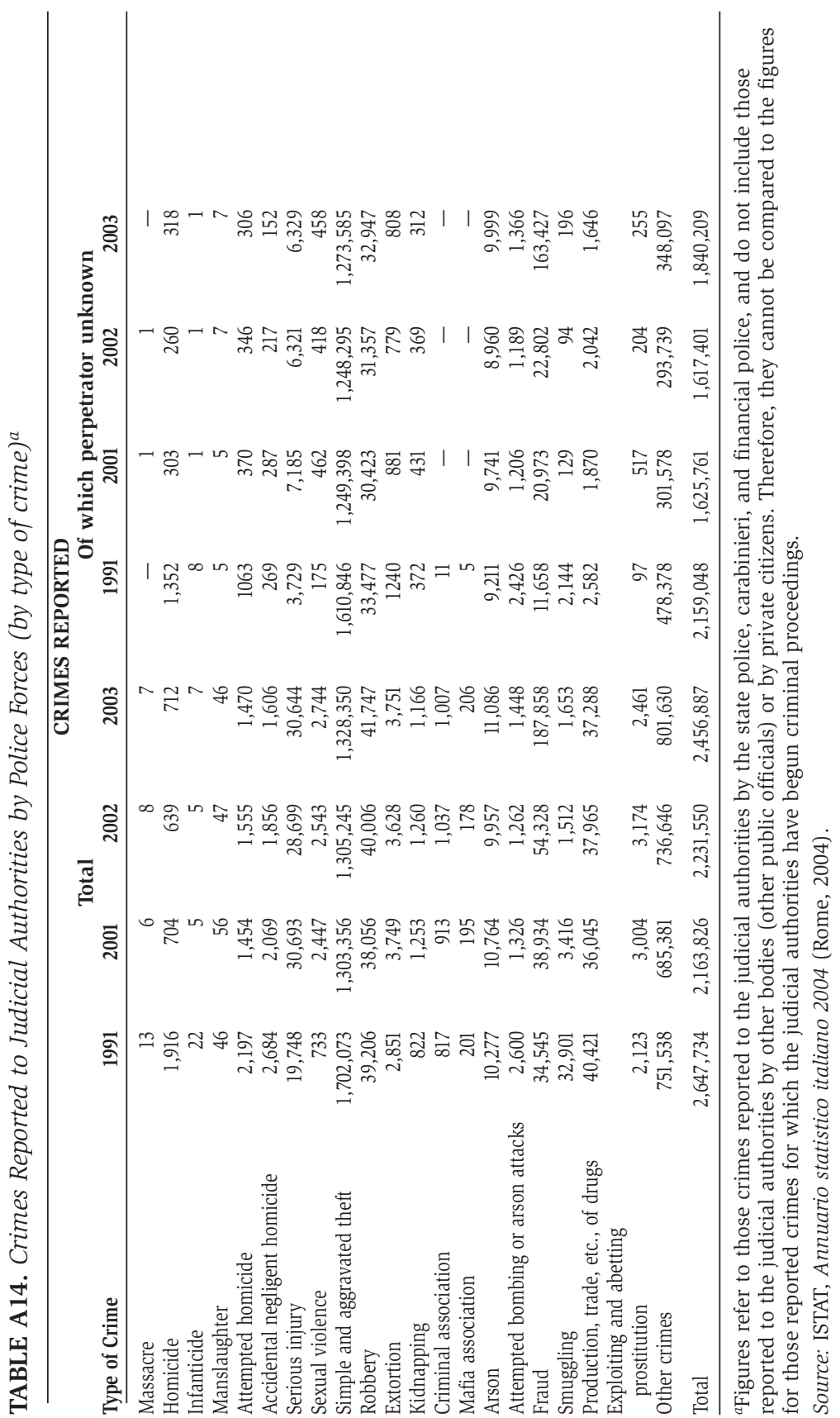




\section{B: ELECTION RESULTS}

Municipal Elections, 2004

TABLE B1a. Turnout at the Municipal Elections of 12 and 13 June 2004 (first ballot, provincial capitals)

\begin{tabular}{|c|c|c|c|c|c|c|c|c|}
\hline $\begin{array}{l}\text { Provincial } \\
\text { capital }\end{array}$ & $\begin{array}{l}\text { Entitled } \\
\text { to vote }\end{array}$ & $\begin{array}{l}\text { \% Voting } \\
\text { (of elec- } \\
\text { torate) }\end{array}$ & Voters & $\begin{array}{c}\% \text { Valid } \\
\text { votes (of } \\
\text { voters) }\end{array}$ & $\begin{array}{l}\% \text { Blank } \\
\text { ballots } \\
\text { (of } \\
\text { voters) }\end{array}$ & $\begin{array}{c}\% \text { Non-valid } \\
\text { or con- } \\
\text { tested } \\
\text { votes (of } \\
\text { voters) }\end{array}$ & $\begin{array}{l}\text { id } \\
\text { Valid } \\
\text { votes }\end{array}$ & $\begin{array}{l}\% \text { Votes } \\
\text { cast for } \\
\text { mayor } \\
\text { alone (of } \\
\text { valid votes) }\end{array}$ \\
\hline Vercelli & 39,629 & 77.7 & 30,806 & 94.2 & 2.1 & 3.7 & 29,023 & 14.1 \\
\hline Biella & 40,227 & 74.5 & 29,968 & 93.2 & 2.9 & 3.9 & 27,934 & 10.4 \\
\hline Verbania & 26,815 & 72.8 & 19,534 & 94.8 & 1.9 & 3.3 & 18,530 & 9.4 \\
\hline Bergamo & 95,914 & 75.1 & 72,056 & 95.4 & 2.2 & 2.4 & 68,776 & 12.3 \\
\hline Cremona & 59,935 & 78.3 & 46,911 & 95.1 & 2.1 & 2.8 & 44,592 & 11.5 \\
\hline Padua & 175,886 & 77.1 & 135,606 & 96.1 & 1.5 & 2.4 & 130,347 & 9.7 \\
\hline Imperia & 34,663 & 80.1 & 27,782 & 96.8 & 1.2 & 2.0 & 26,877 & 5.4 \\
\hline Bologna & 319,529 & 81.8 & 261,471 & 97.1 & 1.2 & 1.7 & 254,088 & 13.7 \\
\hline Ferrara & 116,516 & 81.5 & 94,986 & 95.0 & 2.1 & 2.9 & 90,215 & 8.4 \\
\hline Cesena & 78,851 & 81.7 & 64,449 & 95.4 & 1.8 & 2.8 & 61,498 & 8.3 \\
\hline Forlì & 93,470 & 82.3 & 76,944 & 97.0 & 1.2 & 1.8 & 74,608 & 6.7 \\
\hline Modena & 144,280 & 79.5 & 114,709 & 96.2 & 1.9 & 1.9 & 110,349 & 5.1 \\
\hline Reggio Emilia & 119,766 & 79.8 & 95,547 & 96.0 & 1.8 & 2.2 & 91,685 & 5.9 \\
\hline Arezzo & 77,227 & 80.3 & 62,026 & 96.2 & 1.8 & 2.0 & 59,728 & 7.5 \\
\hline Florence & 307,035 & 76.0 & 233,200 & 95.1 & 2.0 & 2.9 & 221,943 & 7.7 \\
\hline Livorno & 140,322 & 74.3 & 104,321 & 95.6 & 1.7 & 2.7 & 99,732 & 7.0 \\
\hline Prato & 141,983 & 77.1 & 109,470 & 95.0 & 2.3 & 2.7 & 103,994 & 7.1 \\
\hline Perugia & 128,371 & 80.0 & 102,742 & 94.4 & 2.0 & 3.6 & 97,038 & 3.6 \\
\hline Terni & 92,210 & 77.6 & 71,587 & 94.8 & 2.0 & 3.2 & 67,818 & 5.6 \\
\hline Ascoli Piceno & 44,831 & 79.1 & 35,480 & 95.4 & 1.5 & 3.1 & 33,840 & 9.7 \\
\hline Pesaro & 77,422 & 80.2 & 62,115 & 94.5 & 2.1 & 3.4 & 58,693 & 6.6 \\
\hline Urbino & 13,054 & 81.2 & 10,602 & 95.4 & 1.6 & 3.0 & 10,112 & 4.9 \\
\hline Viterbo & 51,766 & 83.3 & 43,137 & 95.8 & 1.5 & 2.7 & 41,347 & 4.8 \\
\hline Teramo & 48,256 & 80.4 & 38,792 & 96.5 & 1.0 & 2.5 & 37,448 & 3.7 \\
\hline Campobasso & 44,430 & 82.3 & 36,562 & 95.7 & 0.7 & 3.6 & 35,025 & 4.0 \\
\hline Avellino & 48,020 & 82.2 & 39,479 & 95.0 & 1.5 & 3.5 & 37,485 & 3.8 \\
\hline Bari & 288,564 & 74.7 & 215,556 & 92.9 & 2.7 & 4.4 & 200,143 & 8.4 \\
\hline Brindisi & 74,729 & 76.0 & 56,807 & 94.7 & 2.1 & 3.2 & 53,835 & 5.5 \\
\hline Foggia & 128,411 & 79.0 & 101,448 & 94.3 & 1.4 & 4.3 & 95,612 & 5.7 \\
\hline Potenza & 58,092 & 84.2 & 48,917 & 94.4 & 1.6 & 4.0 & 46,194 & 3.7 \\
\hline Caltanissetta & 57,107 & 72.9 & 41,645 & 94.5 & 1.4 & 4.1 & 39,375 & 5.6 \\
\hline Siracusa & 103,635 & 77.1 & 79,896 & 93.4 & 2.2 & 4.4 & 74,606 & 5.7 \\
\hline Total & $3,270,946$ & 78.8 & $2,564,551$ & 95.3 & 1.8 & $2.9 \quad 2$ & $2,442,490$ & 7.8 \\
\hline
\end{tabular}

Source: Adapted from data provided by the Ministry of the Interior, Central Election Service. 
TABLE B1b. Turnout at the Municipal Elections of 26 and 27 June 2004 (second ballot, provincial capitals)

\begin{tabular}{|c|c|c|c|c|c|c|c|c|}
\hline $\begin{array}{l}\text { Provincial } \\
\text { capital }\end{array}$ & $\begin{array}{l}\text { Entitled } \\
\text { to vote }\end{array}$ & $\begin{array}{l}\% \text { Voting (of } \\
\text { electorate) }\end{array}$ & Voters & $\begin{array}{l}\% \text { Valid } \\
\text { votes } \\
\text { (of } \\
\text { voters) }\end{array}$ & $\begin{array}{l}\% \text { Blank } \\
\text { ballots } \\
\text { (of } \\
\text { voters) }\end{array}$ & $\begin{array}{l}\% \text { Non- } \\
\text { valid or } \\
\text { contested } \\
\text { votes (of } \\
\text { voters) }\end{array}$ & $\begin{array}{l}\% \text { Diff. in } \\
\text { voting (of } \\
\text { electorate) } \\
\text { between } \\
\text { 1st \& 2nd } \\
\text { ballots }\end{array}$ & $\begin{array}{c}\text { \% Diff. } \\
\text { in valid } \\
\text { votes (of } \\
\text { electorate) } \\
\text { between } \\
\text { 1st \& 2nd } \\
\text { ballots }\end{array}$ \\
\hline Vercelli & 39,629 & 65.7 & 26,027 & 96.8 & 0.9 & 2.3 & -12.1 & -9.7 \\
\hline Biella & 40,227 & 62.2 & 25,023 & 96.6 & 1.2 & 2.2 & -12.3 & -9.3 \\
\hline Bergamo & 95,914 & 60.7 & 58,221 & 98.2 & 0.7 & 1.1 & -14.4 & -12.1 \\
\hline Arezzo & 77,227 & 75.7 & 58,492 & 98.8 & 0.5 & 0.7 & -4.6 & -2.6 \\
\hline Florence & 307,035 & 51.9 & 159,202 & 97.3 & 0.9 & 1.8 & -24.1 & -21.8 \\
\hline Foggia & 128,411 & 59.3 & 76,113 & 98.2 & 0.4 & 1.4 & -19.7 & -16.3 \\
\hline Total & 688,443 & 58.5 & 403,078 & 97.7 & 0.8 & 1.5 & -18.4 & -15.9 \\
\hline
\end{tabular}

Source: Adapted from data provided by the Ministry of the Interior, Central Election Service. 


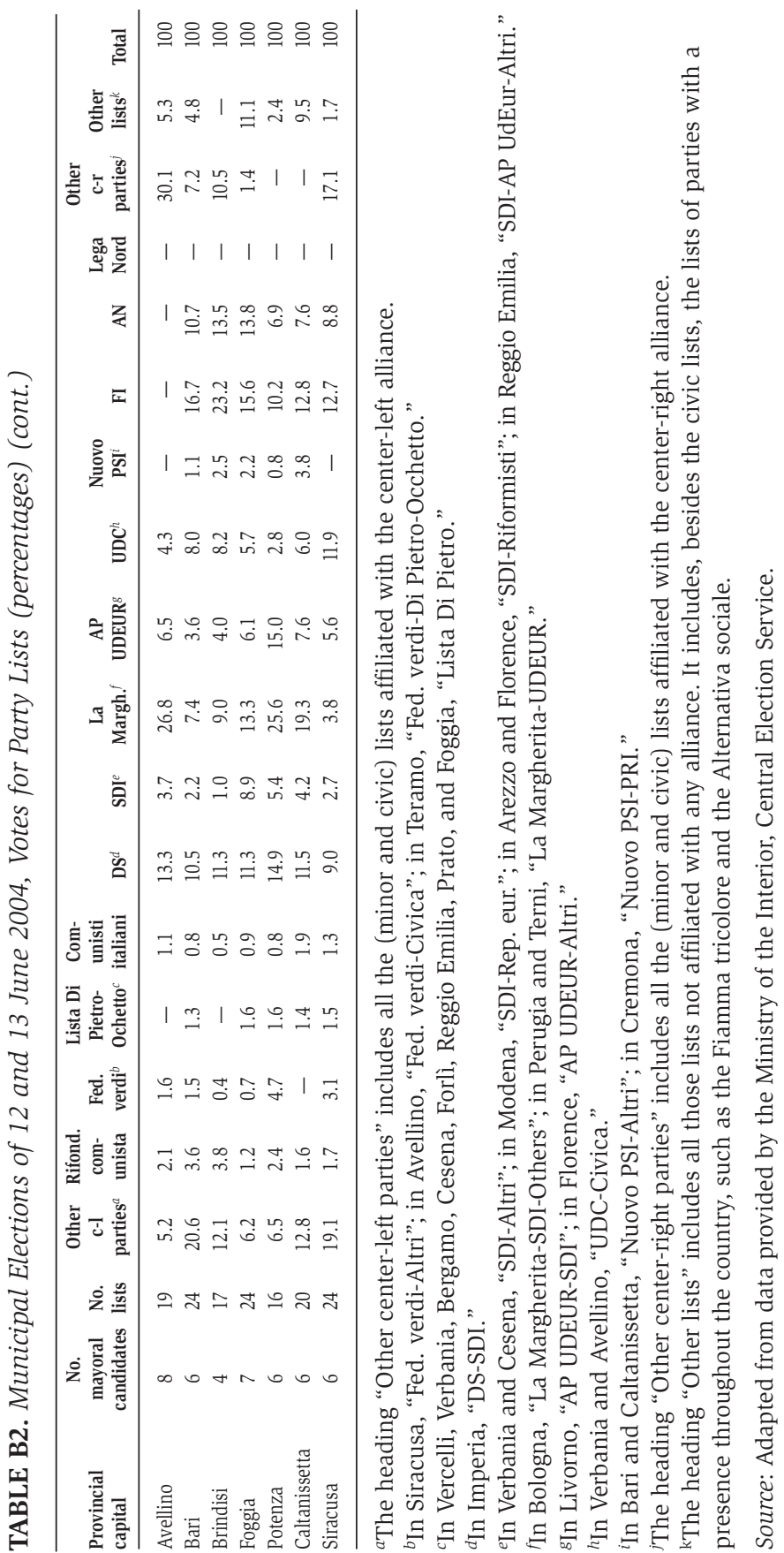




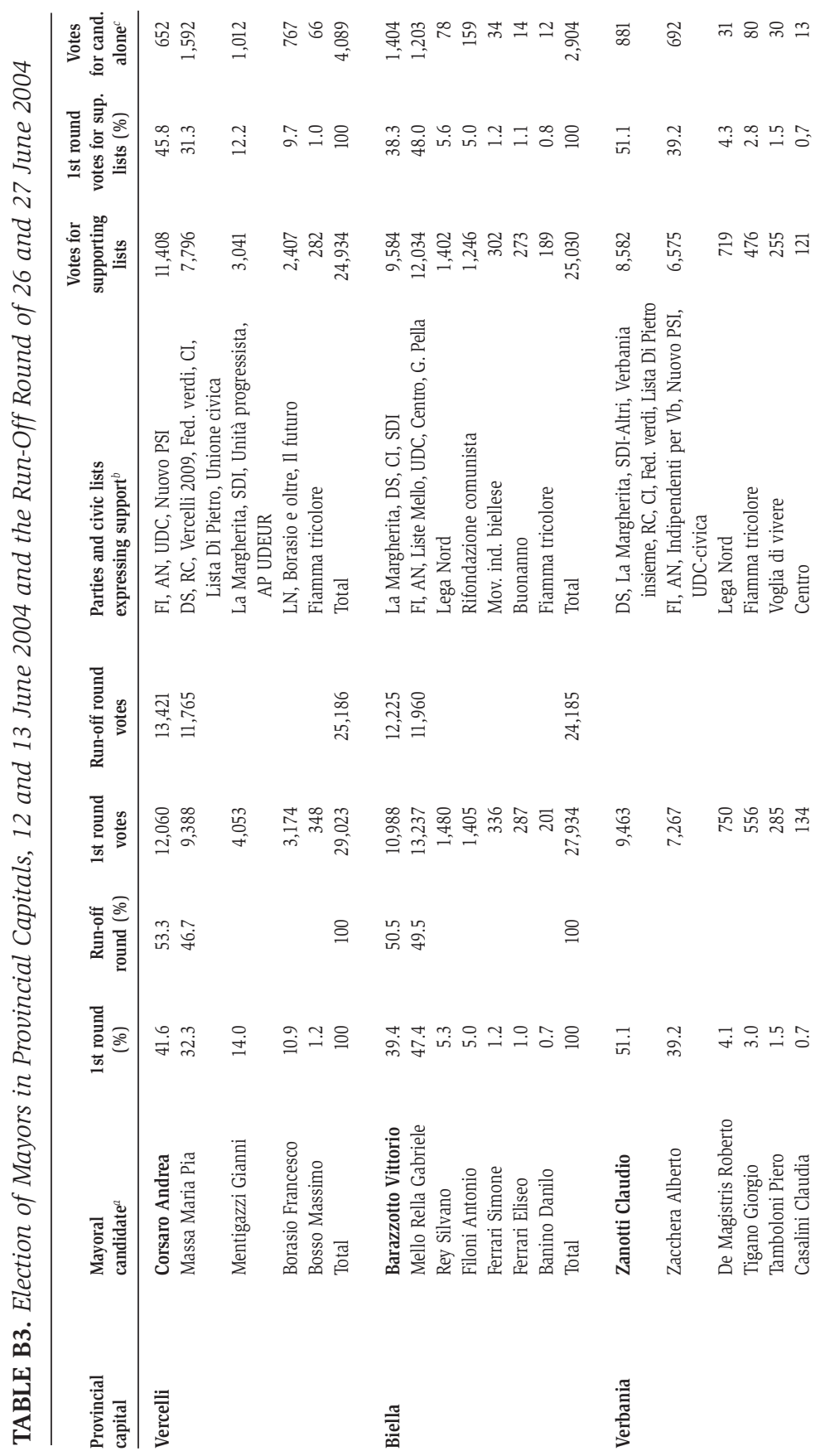




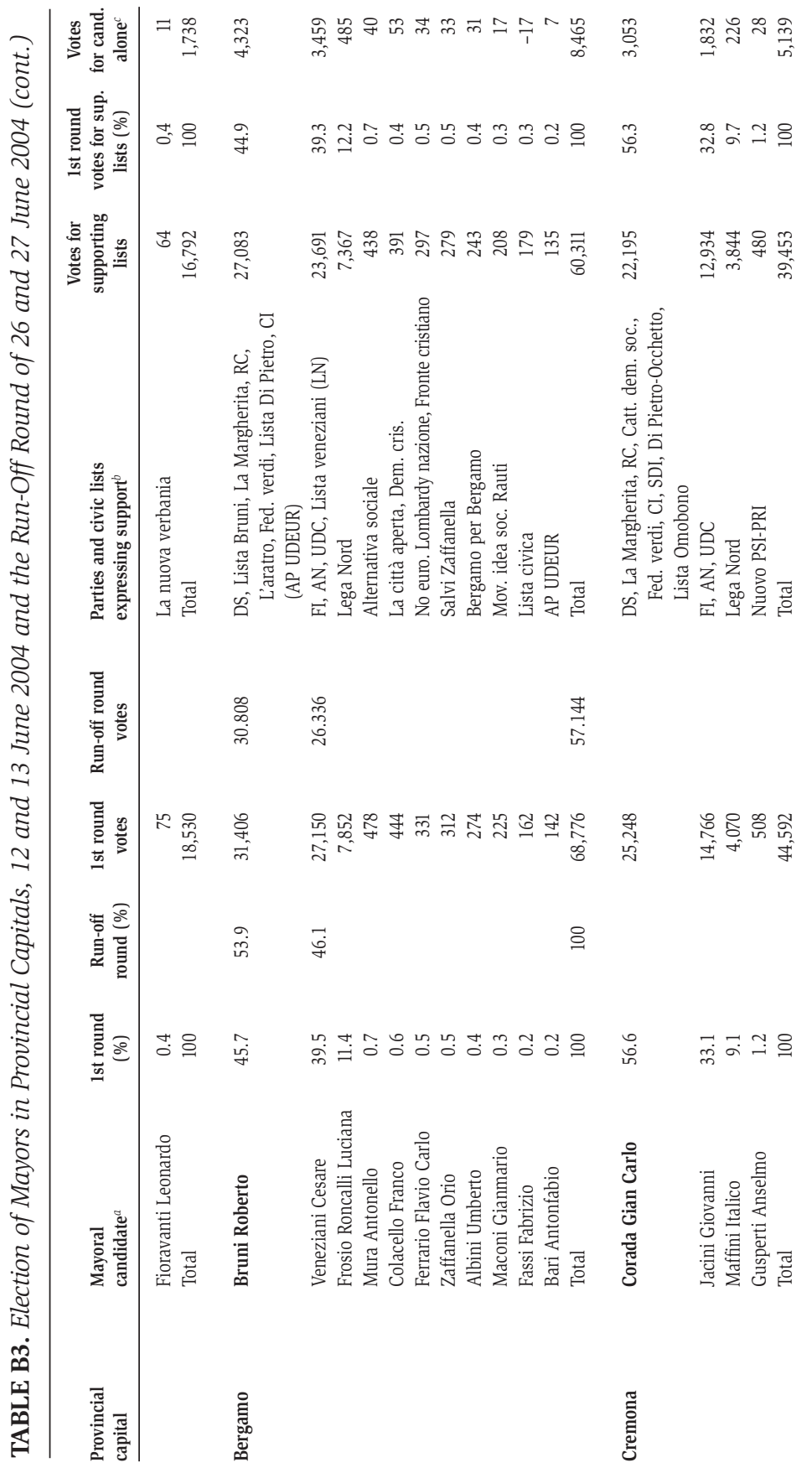




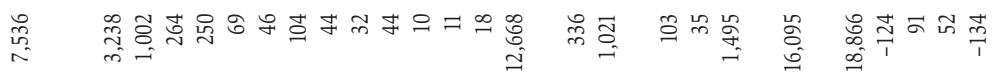

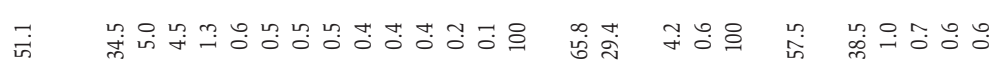

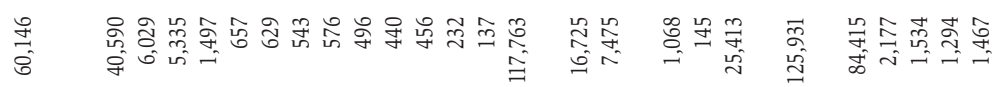
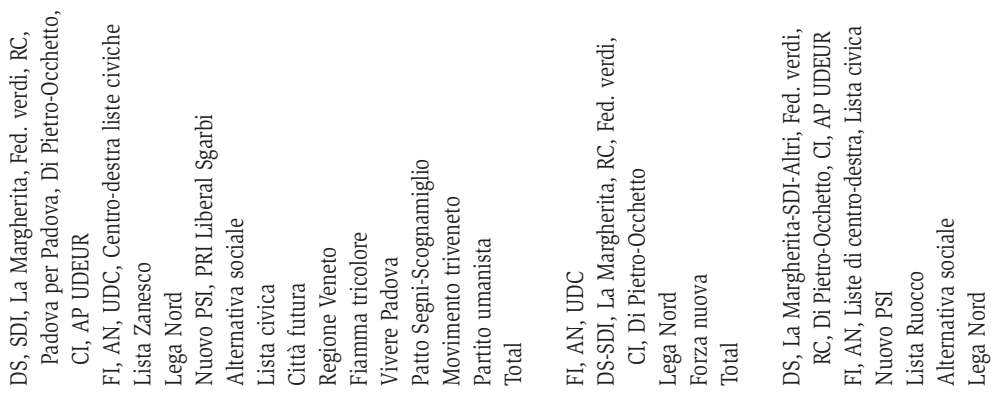

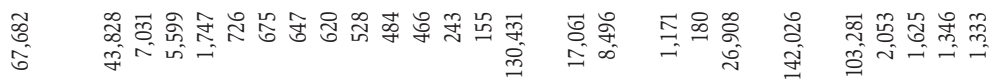

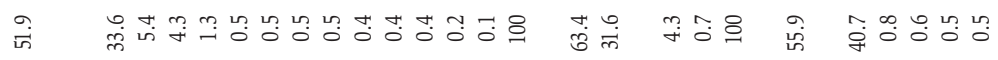

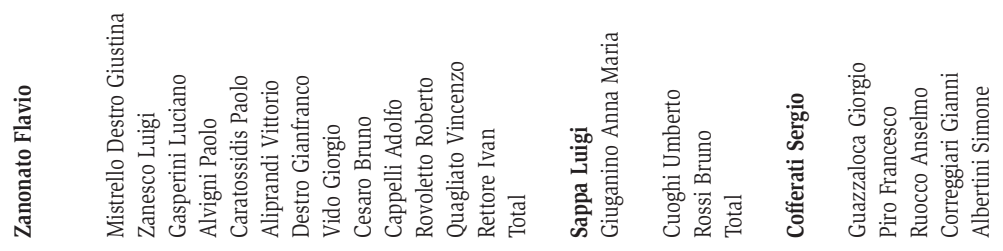

$\stackrel{\Xi}{\overparen{Z}}$

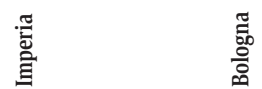




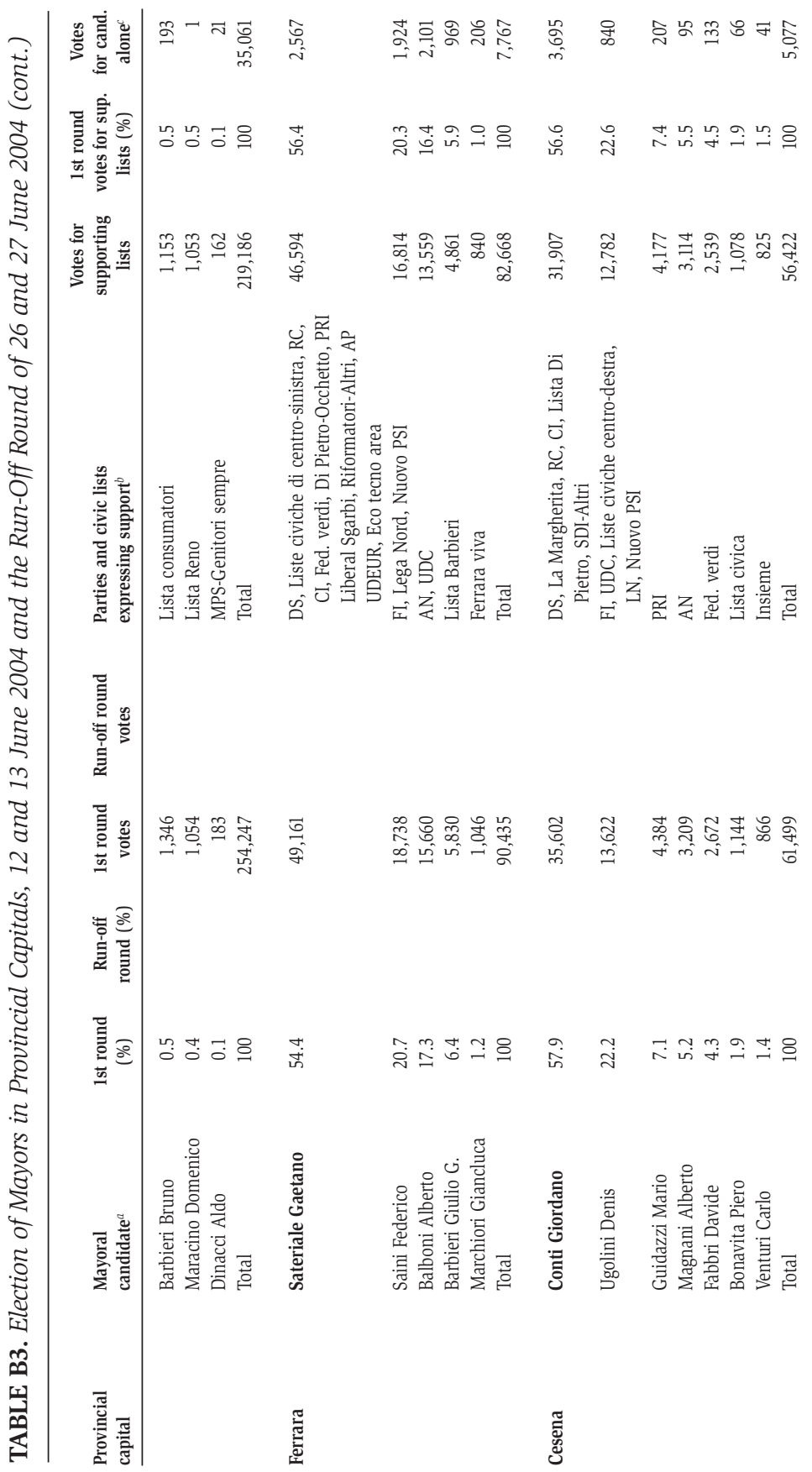




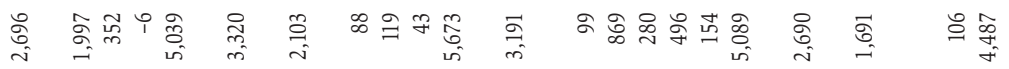
ऊ

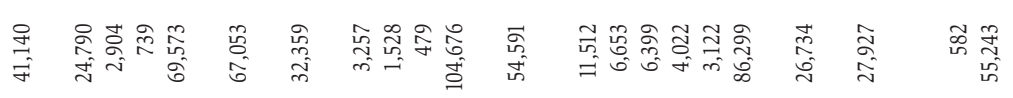

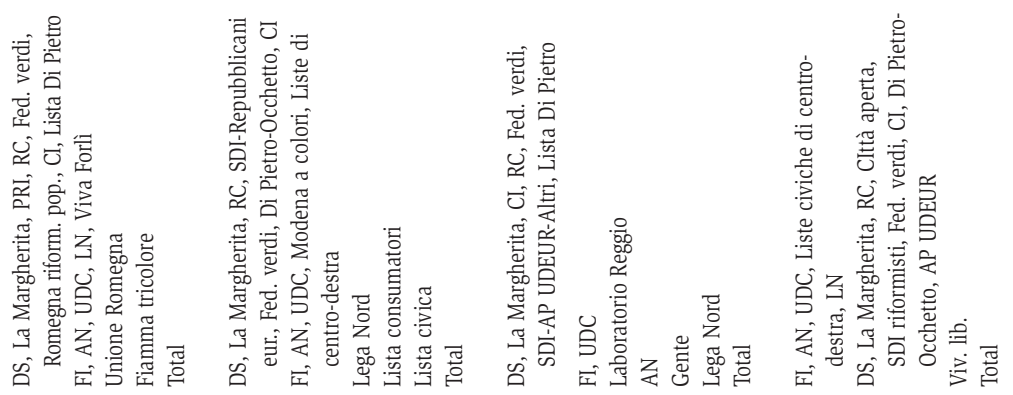

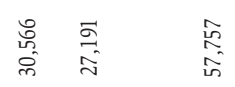

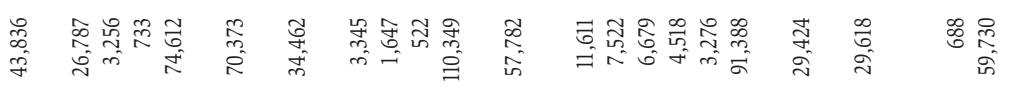

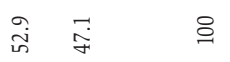

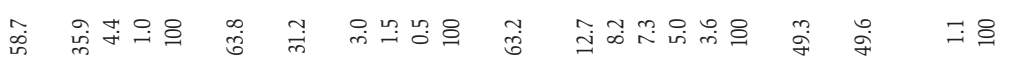

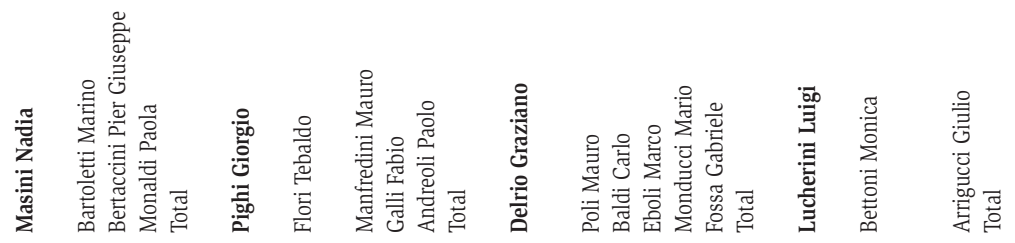
올 


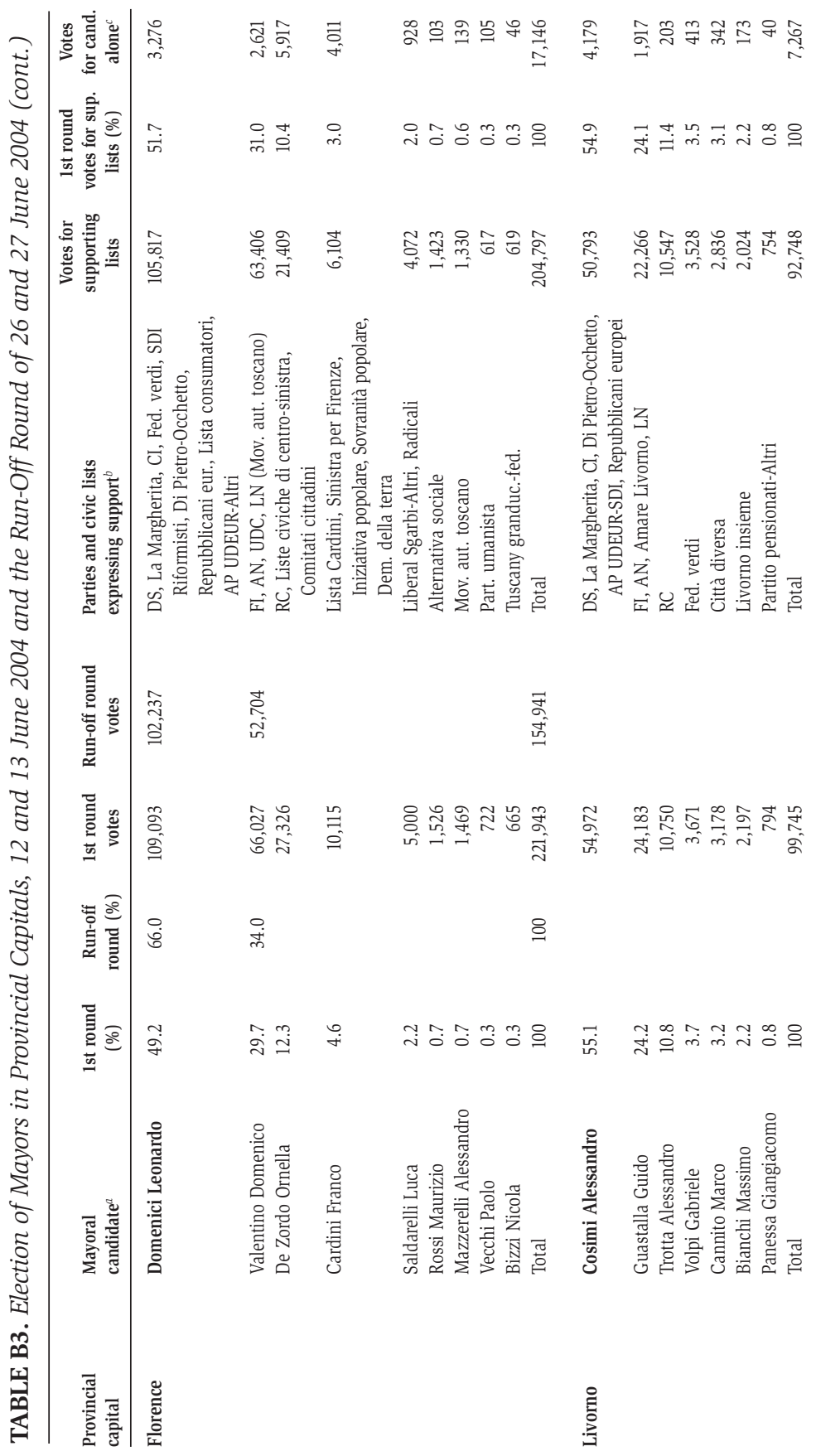




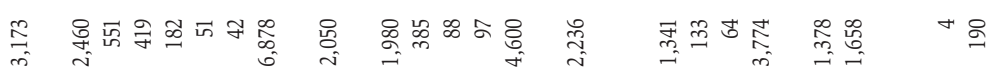

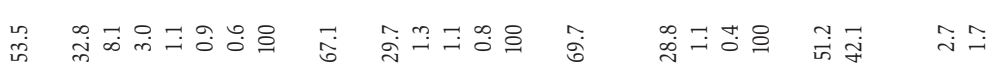

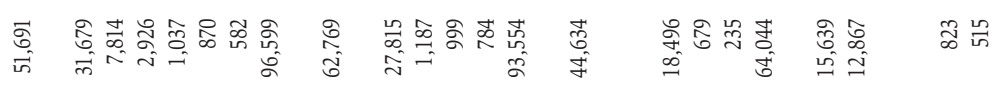

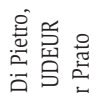

焉安

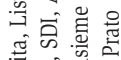
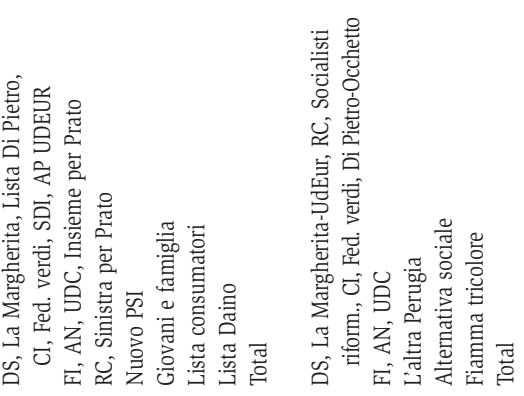

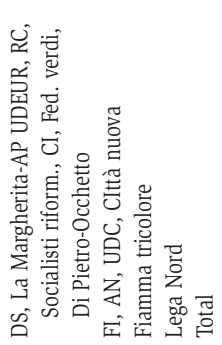

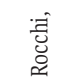

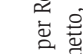

Ð艹

फ $\frac{1}{3}$

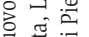

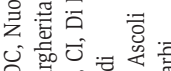

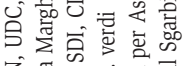

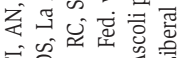

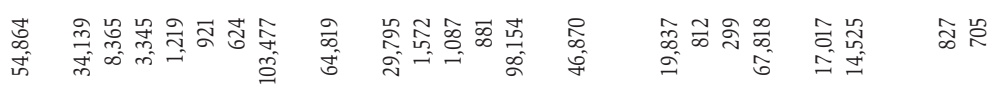

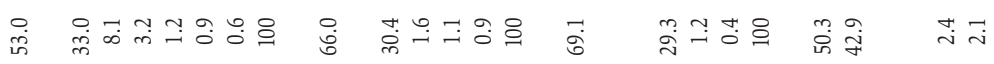

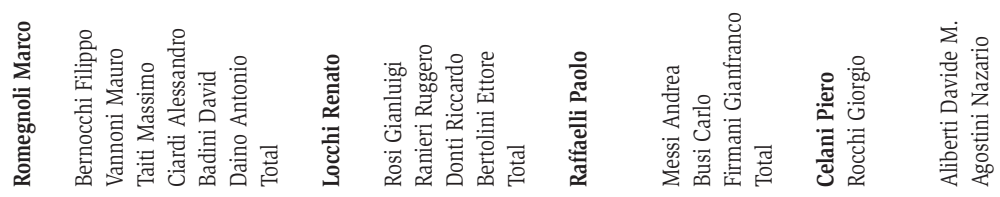

을

물

芜

물 


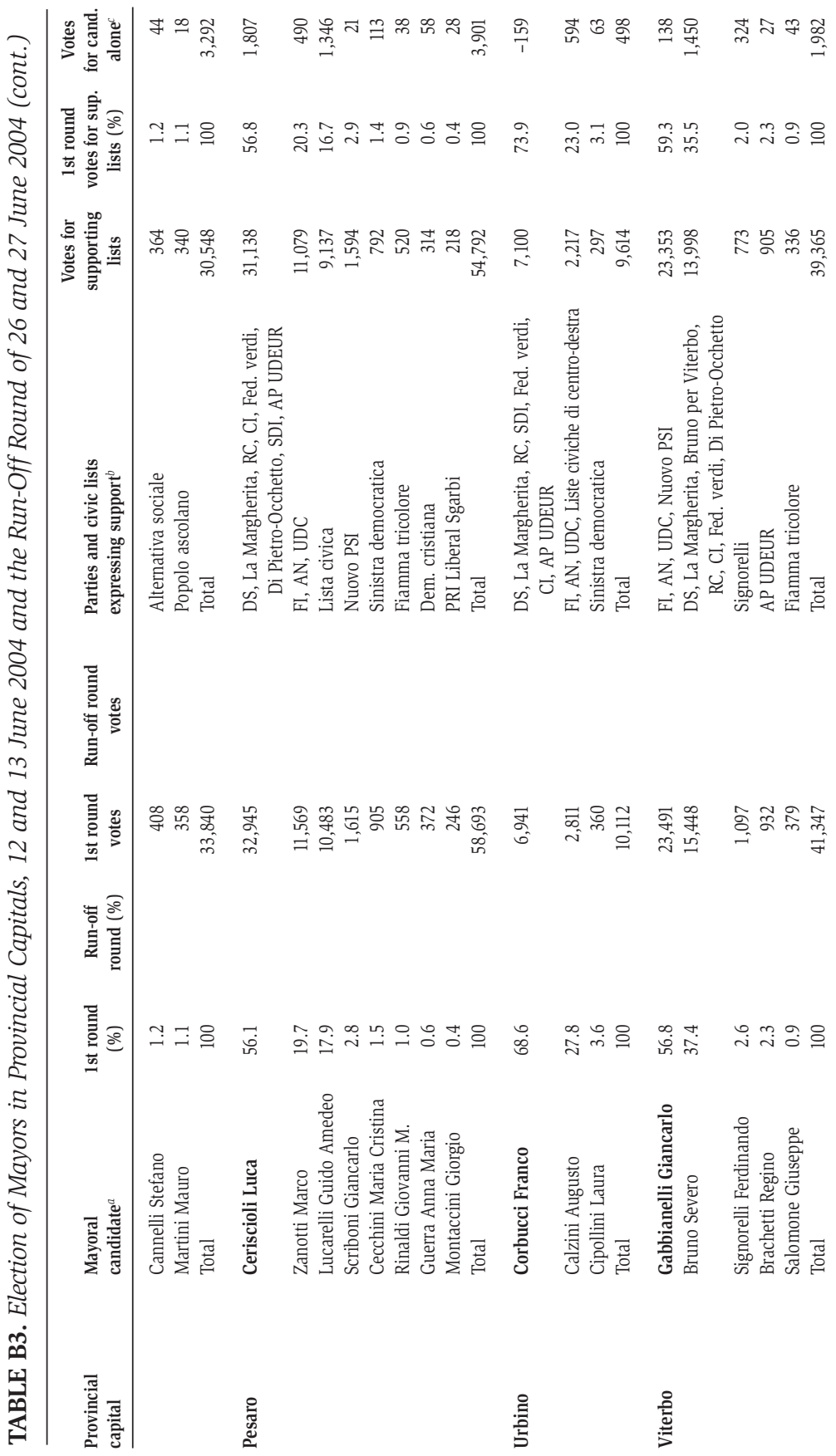




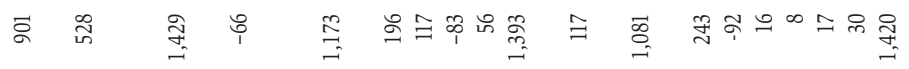

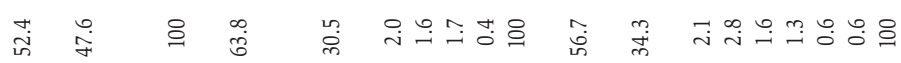

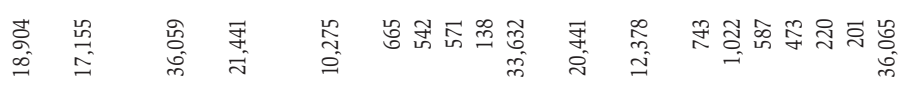

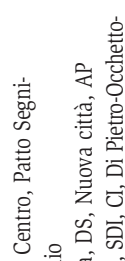

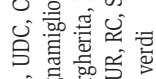

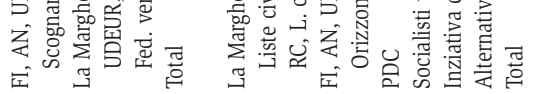

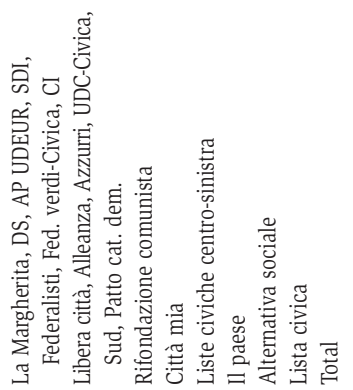

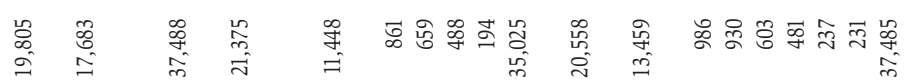

๙

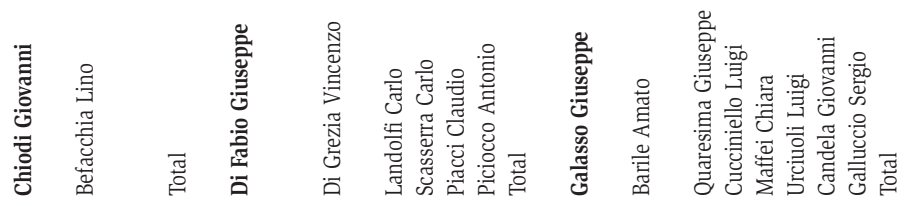

总

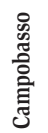

竞 


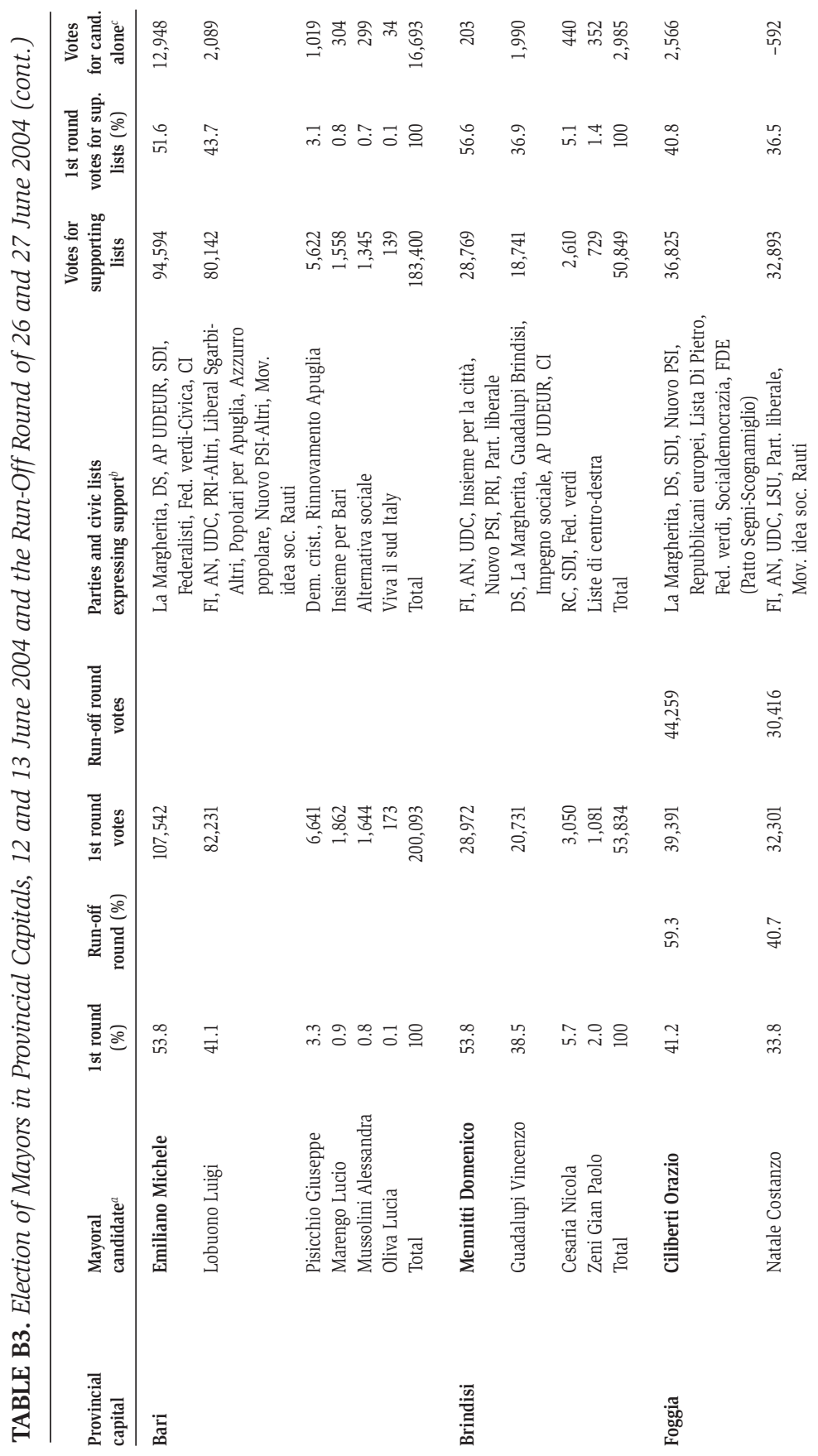




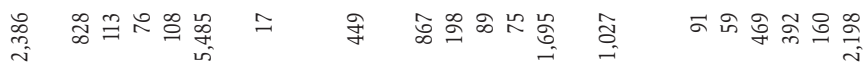

શે

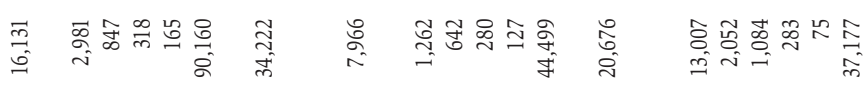

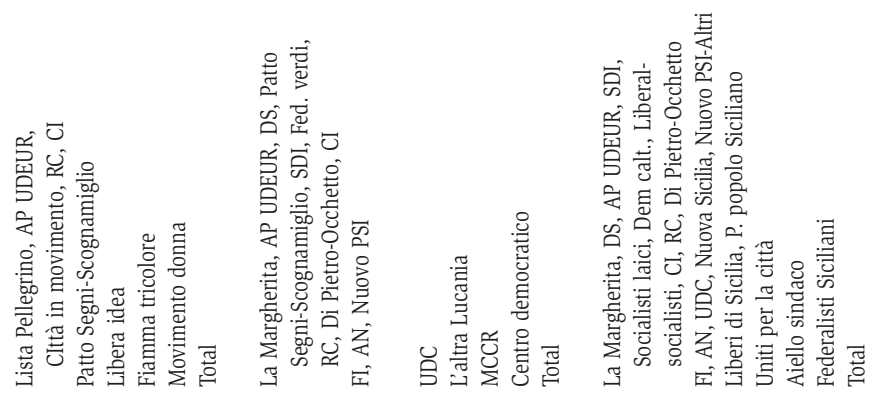

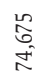

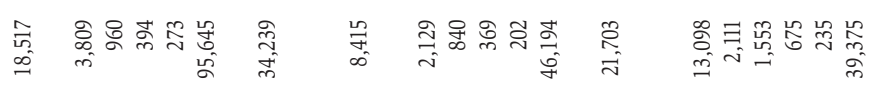

\&

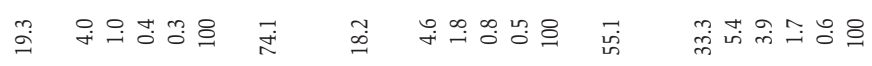

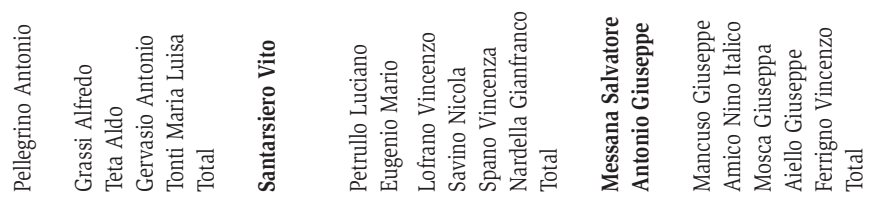

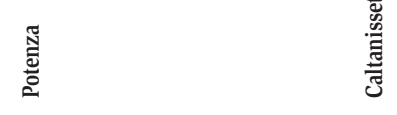




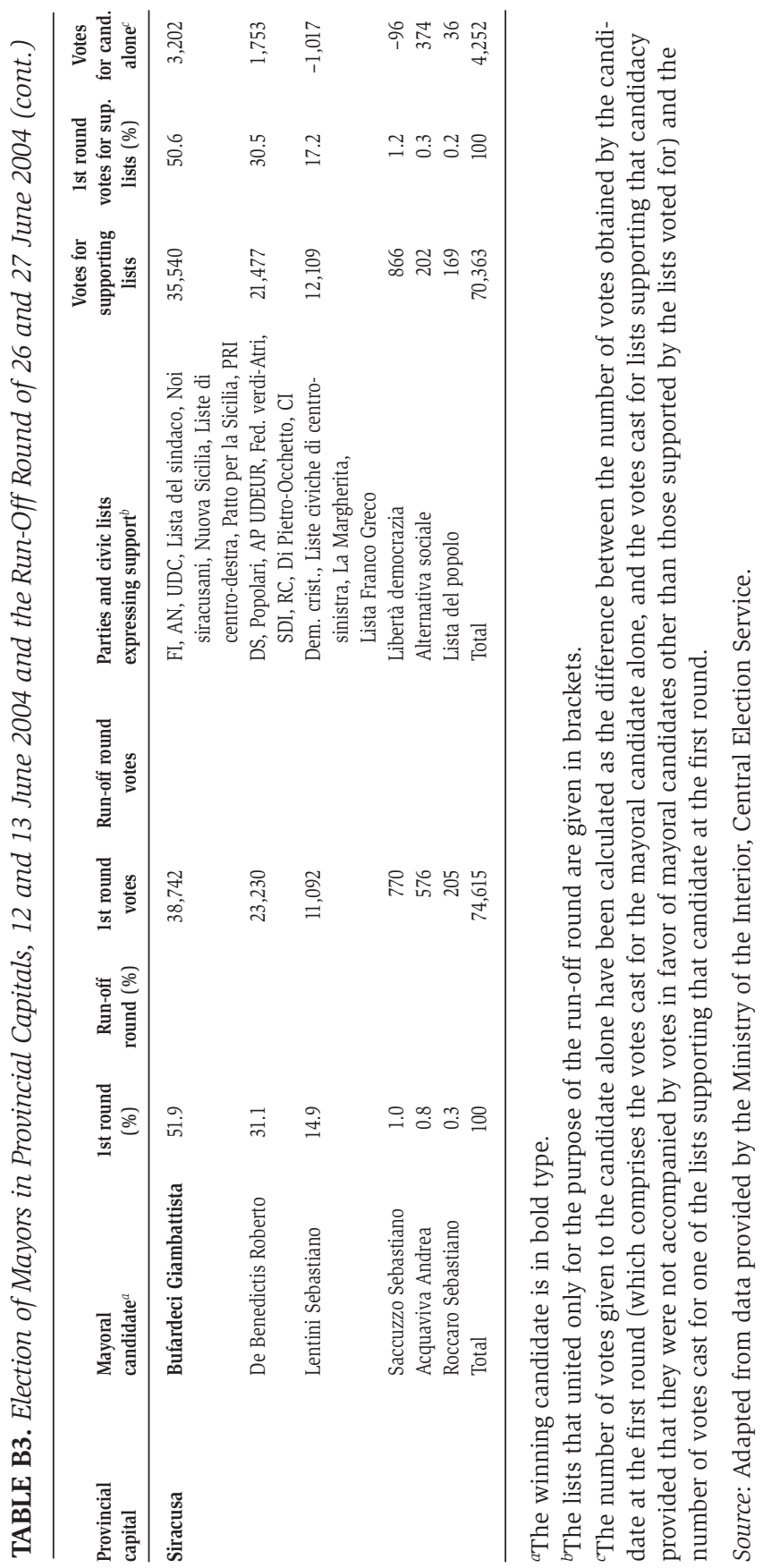


Provincial Elections, 2004

TABLE B4a. Turnout at the Provincial Elections of 12 and 13 June 2004 (first round)

\begin{tabular}{|c|c|c|c|c|c|c|c|c|}
\hline Province & $\begin{array}{l}\text { Entitled } \\
\text { to vote }\end{array}$ & $\begin{array}{l}\text { \% Voting } \\
\text { (of elec- } \\
\text { torate) }\end{array}$ & Voters & $\begin{array}{c}\% \text { Valid } \\
\text { votes (of } \\
\text { voters) }\end{array}$ & $\begin{array}{l}\% \text { Blank } \\
\text { ballots } \\
\text { (of } \\
\text { voters) }\end{array}$ & $\begin{array}{c}\% \text { Non-valid } \\
\text { or con- } \\
\text { tested } \\
\text { votes (of } \\
\text { voters) }\end{array}$ & $\begin{array}{l}\text { Valid } \\
\text { votes }\end{array}$ & $\begin{array}{c}\% \text { Votes } \\
\text { cast for } \\
\text { president } \\
\text { alone (of } \\
\text { valid votes) }\end{array}$ \\
\hline Pordenone & 268,315 & 67.6 & 181,333 & 93.6 & 3.3 & 3.1 & 169,670 & 11.4 \\
\hline Alessandria & 373,532 & 74.9 & 279,946 & 90.6 & 5.3 & 4.1 & 253,637 & 10.0 \\
\hline Asti & 179,960 & 76.0 & 136,790 & 89.8 & 6.2 & 4.0 & 122,848 & 13.2 \\
\hline Cuneo & 473,847 & 78.4 & 371,713 & 86.4 & 7.4 & 6.2 & 321,071 & 10.5 \\
\hline Novara & 294,076 & 76.0 & 223,484 & 89.6 & 5.6 & 4.8 & 200,147 & 11.8 \\
\hline Turin & $1,875,998$ & 72.8 & $1,365,456$ & 90.3 & 4.7 & 5.0 & $1,233,462$ & 10.4 \\
\hline Biella & 163,371 & 78.1 & 127,572 & 90.4 & 5.3 & 4.3 & 115,357 & 14.2 \\
\hline \multicolumn{9}{|l|}{ Verbano-Cusio- } \\
\hline Ossola & 144,742 & 73.1 & 105,820 & 91.4 & 4.6 & 4.0 & 96,741 & 10.8 \\
\hline Bergamo & 818,374 & 78.4 & 641,403 & 90.5 & 5.8 & 3.7 & 580,501 & 10.2 \\
\hline Brescia & 916,070 & 100.0 & 916,070 & 73.2 & 4.4 & 22.4 & 670,201 & 12.9 \\
\hline Cremona & 284,330 & 80.5 & 228,802 & 90.1 & 5.8 & 4.1 & 206,197 & 9.6 \\
\hline Milan & $3,108,448$ & 73.32 & $2,277,706$ & 92.4 & 3.8 & 3.8 & $2,104,619$ & 13.0 \\
\hline Sondrio & 158,327 & 72.0 & 113,927 & 90.0 & 5.8 & 4.2 & 102,625 & 14.2 \\
\hline Lecco & 260,980 & 78.5 & 204,938 & 92.3 & 4.1 & 3.6 & 189,303 & 9.0 \\
\hline Lodi & 167,020 & 80.7 & 134,751 & 91.7 & 5.7 & 2.6 & 123,584 & 7.6 \\
\hline Belluno & 202,257 & 64.2 & 129,795 & 93.7 & 3.0 & 3.3 & 121,696 & 15.0 \\
\hline Padua & 728,059 & 80.0 & 582,343 & 91.4 & 4.6 & 4.0 & 532,600 & 8.9 \\
\hline Rovigo & 211,556 & 79.7 & 168,662 & 90.1 & 6.2 & 3.7 & 152,012 & 6.8 \\
\hline Venice & 706,270 & 73.0 & 515,331 & 89.8 & 0.9 & 9.3 & 462,648 & 4.8 \\
\hline Verona & 688,384 & 77.0 & 530,171 & 92.1 & 4.1 & 3.8 & 488,525 & 9.7 \\
\hline Savona & 247,257 & 79.3 & 196,177 & 87.9 & 7.3 & 4.8 & 172,424 & 4.7 \\
\hline Bologna & 785,226 & 82.9 & 651,259 & 94.0 & 3.6 & 2.4 & 612,431 & 5.3 \\
\hline Ferrara & 306,300 & 81.3 & 249,075 & 92.4 & 3.9 & 3.7 & 230,178 & 7.2 \\
\hline Forlì-Cesena & 309,406 & 81.6 & 252,568 & 93.7 & 3.4 & 2.9 & 236,618 & 4.3 \\
\hline Modena & 530,958 & 80.5 & 427,303 & 94.4 & 3.7 & 1.9 & 403,432 & 4.3 \\
\hline Parma & 349,767 & 76.2 & 266,512 & 94.4 & 3.1 & 2.5 & 251,630 & 13.3 \\
\hline Piacenza & 232,595 & 76.7 & 178,367 & 91.8 & 4.3 & 3.9 & 163,786 & 14.3 \\
\hline Reggio Emilia & 384,698 & 81.5 & 313,568 & 93.9 & 3.8 & 2.3 & 294,588 & 5.1 \\
\hline Rimini & 238,387 & 77.1 & 183,781 & 93.3 & 3.5 & 3.2 & 171,486 & 6.5 \\
\hline Arezzo & 273,694 & 80.4 & 220,052 & 91.9 & 5.3 & 2.8 & 202,167 & 5.0 \\
\hline Florence & 792,155 & 79.5 & 629,461 & 94.0 & 3.4 & 2.6 & 591,526 & 4.7 \\
\hline Grosseto & 185,480 & 78.8 & 146,209 & 92.9 & 3.9 & 3.2 & 135,781 & 10.6 \\
\hline Livorno & 288,943 & 76.8 & 221,981 & 93.0 & 4.1 & 2.9 & 206,542 & 4.7 \\
\hline Pisa & 329,342 & 78.3 & 257,934 & 93.5 & 3.6 & 2.9 & 241,121 & 5.8 \\
\hline Pistoia & 234,577 & 76.9 & 180,337 & 91.8 & 4.3 & 3.9 & 165,692 & 5.9 \\
\hline Siena & 215,599 & 80.4 & 173,285 & 92.9 & 4.3 & 2.8 & 161,054 & 4.5 \\
\hline Prato & 189,116 & 77.5 & 146,477 & 91.8 & 3.6 & 4.6 & 134,376 & 5.5 \\
\hline Perugia & 525,287 & 78.7 & 413,382 & 92.0 & 4.7 & 3.3 & 380,438 & 2.6 \\
\hline Terni & 190,904 & 79.7 & 152,154 & 91.4 & 4.9 & 3.7 & 139,163 & 4.9 \\
\hline Ascoli Piceno & 322,084 & 76.9 & 247,591 & 90.4 & 6.1 & 3.5 & 223,791 & 7.3 \\
\hline Macerata & 264,088 & 75.9 & 200,400 & 90.8 & 5.9 & 3.3 & 181,895 & 12.3 \\
\hline Pesaro and Urbino & 308,230 & 79.5 & 245,051 & 91.4 & 5.4 & 3.2 & 223,942 & 6.3 \\
\hline Frosinone & 447,603 & 74.5 & 333,406 & 91.7 & 4.4 & 3.9 & 305,789 & 2.2 \\
\hline Latina & 446,424 & 73.8 & 329,369 & 92.1 & 3.6 & 4.3 & 303,388 & 3.3 \\
\hline
\end{tabular}


TABLE B4a. Turnout at the Provincial Elections of 12 and 13 June 2004 (first round) (cont.)

\begin{tabular}{|c|c|c|c|c|c|c|c|c|}
\hline Province & $\begin{array}{l}\text { Entitled } \\
\text { to vote }\end{array}$ & $\begin{array}{l}\% \text { Voting } \\
\text { (of elec- } \\
\text { torate) }\end{array}$ & Voters & $\begin{array}{c}\% \text { Valid } \\
\text { votes (of } \\
\text { voters) }\end{array}$ & $\begin{array}{c}\% \text { Blank } \\
\text { ballots } \\
\text { (of } \\
\text { voters) }\end{array}$ & $\begin{array}{l}\% \text { Non-valid } \\
\text { or con- } \\
\text { tested } \\
\text { votes (of } \\
\text { voters) }\end{array}$ & $\begin{array}{l}\text { id } \\
\text { Valid } \\
\text { votes }\end{array}$ & $\begin{array}{c}\% \text { Votes } \\
\text { cast for } \\
\text { president } \\
\text { alone (of } \\
\text { valid votes) }\end{array}$ \\
\hline Rieti & 128,740 & 82.8 & 106,547 & 94.4 & 3.3 & 2.3 & 100,604 & 7.7 \\
\hline Chieti & 376,186 & 69.1 & 259,880 & 91.3 & 4.5 & 4.2 & 237,208 & 4.3 \\
\hline L'Aquila & 277,681 & 72.7 & 201,924 & 90.7 & 4.9 & 4.4 & 183,158 & 5.1 \\
\hline Pescara & 280,541 & 70.5 & 197,689 & 92.9 & 3.6 & 3.5 & 183,665 & 6.8 \\
\hline Teramo & 264,458 & 75.5 & 199,657 & 91.0 & 5.6 & 3.4 & 181,755 & 4.1 \\
\hline Isernia & 93,150 & 66.9 & 62,304 & 90.2 & 5.5 & 4.3 & 56,211 & 4.3 \\
\hline Avellino & 428,533 & 68.8 & 294,698 & 92.8 & 6.0 & 1.2 & 273,466 & 4.4 \\
\hline Naples & $2,490,238$ & 64.3 & $1,600,480$ & 92.1 & 3.9 & 4.0 & $1,473,012$ & 2.5 \\
\hline Salerno & 941,485 & 74.2 & 698,163 & 90.8 & 4.5 & 4.7 & 634,028 & 2.7 \\
\hline Bari & $1,356,437$ & 68.6 & 930,790 & 89.1 & 5.7 & 5.2 & 829,148 & 5.1 \\
\hline Brindisi & 347,760 & 72.7 & 252,981 & 89.0 & 5.7 & 5.3 & 225,228 & 5.7 \\
\hline Lecce & 720,511 & 73.2 & 527,133 & 92.1 & 4.3 & 3.6 & 485,372 & 2.5 \\
\hline Taranto & 497,483 & 70.1 & 348,924 & 91.3 & 4.0 & 4.7 & 318,527 & 4.6 \\
\hline Matera & 176,830 & 72.0 & 127,337 & 89.4 & 3.9 & 6.7 & 113,818 & 2.8 \\
\hline Potenza & 374,274 & 70.7 & 264,535 & 88.5 & 6.5 & 5.0 & 234,151 & 1.7 \\
\hline Catanzaro & 339,906 & 65.8 & 223,689 & 92.1 & 3.8 & 4.1 & 206,102 & 2.4 \\
\hline Cosenza & 676,176 & 68.0 & 459,682 & 94.6 & 5.4 & 0.0 & 435,018 & 5.8 \\
\hline Crotone & 150,585 & 65.9 & 101,805 & 91.6 & 2.9 & 5.5 & 93,174 & 2.7 \\
\hline Vibo Valentia & 165,409 & 65.5 & 108,291 & 91.8 & 4.7 & 3.5 & 99,331 & 1.3 \\
\hline Total & $30,508,419$ & 74.82 & $22,815,716$ & 90.9 & 4.5 & $4.6 \quad 20$ & $20,743,658$ & 7.2 \\
\hline
\end{tabular}

Source: Adapted from data provided by the Ministry of the Interior, Central Election Service. 
TABLE B4b. Turnout at the Provincial Elections of 26 and 27 June 2004 (second round)

\begin{tabular}{|c|c|c|c|c|c|c|c|c|}
\hline Province & $\begin{array}{l}\text { Entitled } \\
\text { to vote }\end{array}$ & $\begin{array}{l}\% \text { Voting } \\
\text { (of elec- } \\
\text { torate) }\end{array}$ & Voters & $\begin{array}{c}\% \text { Valid } \\
\text { votes (of } \\
\text { voters) }\end{array}$ & $\begin{array}{l}\% \text { Blank } \\
\text { ballots } \\
\text { of voters) }\end{array}$ & $\begin{array}{l}\% \text { Non-valid } \\
\text { or con- } \\
\text { tested } \\
\text { votes (of } \\
\text { voters) }\end{array}$ & $\begin{array}{l}\% \text { Diff. in } \\
\text { voting } \\
\text { (of elec.) } \\
\text { betw. 1st \& } \\
\text { 2nd ballots }\end{array}$ & $\begin{array}{l}\% \text { Diff. in } \\
\text { valid votes } \\
\text { (of elec.) } \\
\text { betw. 1st \& } \\
\text { 2nd ballots }\end{array}$ \\
\hline Pordenone & 268,315 & 45.6 & 119,765 & 97.3 & 0.8 & 1.9 & -22.9 & -19.8 \\
\hline Novara & 294,076 & 48.5 & 142,591 & 96.8 & 1.1 & 2.2 & -27.5 & -21.1 \\
\hline Biella & 163,371 & 58.3 & 95,237 & 96.5 & 1.3 & 2.2 & -19.8 & -14.3 \\
\hline \multicolumn{9}{|c|}{ Verbano-Cusio- } \\
\hline Ossola & 144,742 & 50.5 & 73,032 & 97.5 & 0.8 & 1.7 & -22.7 & -17.7 \\
\hline Bergamo & 818,374 & 47.6 & 389,882 & 95.8 & 1.8 & 2.4 & -30.7 & -25.3 \\
\hline Brescia & 916,070 & 53.7 & 491,526 & 97.1 & 1.0 & 1.9 & -46.3 & -21.0 \\
\hline Cremona & 284,330 & 54.5 & 154,875 & 97.1 & 1.1 & 1.8 & -26.0 & -19.6 \\
\hline Milan & $3,108,448$ & 53.01 & $1,646,438$ & 98.0 & 0.6 & 1.4 & -20.3 & -15.8 \\
\hline Sondrio & 158,327 & 36.6 & 57,938 & 94.9 & 2.2 & 2.9 & -35.4 & -30.1 \\
\hline Lecco & 260,980 & 51.5 & 134,380 & 97.4 & 0.9 & 1.8 & -27.0 & -22.4 \\
\hline Lodi & 167,020 & 54.4 & 90,936 & 96.9 & 1.0 & 2.1 & -26.2 & -21.2 \\
\hline Belluno & 202,257 & 47.3 & 95,607 & 98.1 & 0.7 & 1.2 & -16.9 & -13.8 \\
\hline Padua & 728,059 & 54.9 & 400,009 & 97.2 & 1.0 & 1.8 & -25.0 & -19.7 \\
\hline Verona & 688,384 & 51.7 & 356,129 & 97.6 & 0.9 & 1.5 & -25.3 & -20.5 \\
\hline Piacenza & 232,595 & 60.9 & 141,738 & 97.3 & 1.0 & 1.7 & -15.7 & -11.1 \\
\hline Macerata & 264,088 & 56.8 & 150,067 & 97.4 & 1.1 & 1.5 & -19.1 & -13.6 \\
\hline Rieti & 128,740 & 67.7 & 87,164 & 97.8 & 0.7 & 1.5 & -15.1 & -11.9 \\
\hline Chieti & 376,186 & 56.3 & 211,628 & 97.1 & 1.2 & 1.7 & -12.8 & -8.4 \\
\hline L'Aquila & 277,681 & 52.6 & 146,075 & 97.1 & 1.2 & 1.7 & -20.1 & -14.9 \\
\hline Isernia & 93,150 & 50.4 & 46,928 & 96.2 & 1.8 & 2.0 & -16.5 & -11.9 \\
\hline Brindisi & 347,760 & 52.0 & 180,980 & 96.5 & 1.2 & 2.3 & -20.7 & -14.6 \\
\hline Catanzaro & 339,906 & 47.6 & 161,801 & 96.6 & 1.2 & 2.2 & -18.2 & -14.7 \\
\hline Total & $10,262,859$ & 52.45 & $5,374,276$ & 97.3 & 1.0 & 1.7 & -24.7 & -18.0 \\
\hline
\end{tabular}

Source: Adapted from data provided by the Ministry of the Interior, Central Election Service. The data for the province of Pordenone were drawn from the official Web site of the Friuli-Venezia Giulia regional council. 


\begin{tabular}{|c|c|c|}
\hline 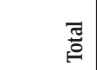 & ㅇㅇㅇ @ @ & 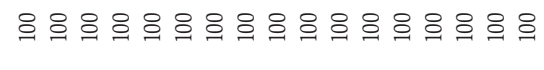 \\
\hline 总: & 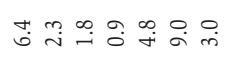 & 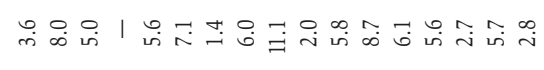 \\
\hline 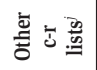 & 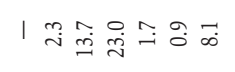 & 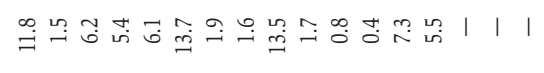 \\
\hline 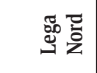 & 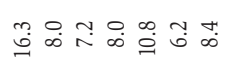 & 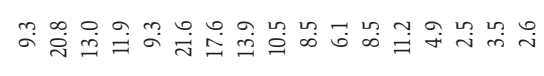 \\
\hline 玄 & 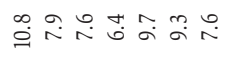 & 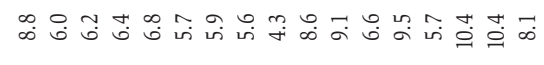 \\
\hline 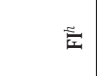 & 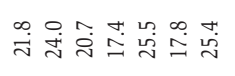 & 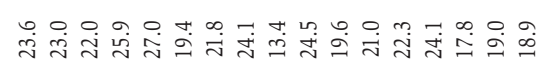 \\
\hline 总总 & | & 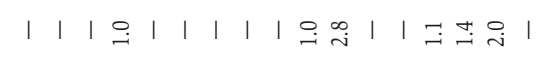 \\
\hline 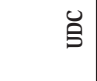 & 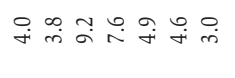 & 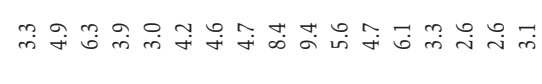 \\
\hline を善 & 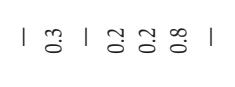 & 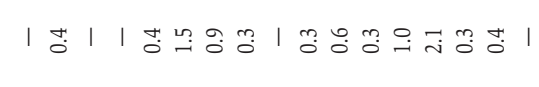 \\
\hline 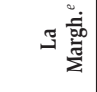 & 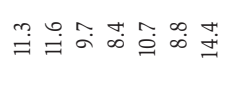 & 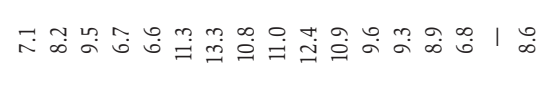 \\
\hline 苨 & $\stackrel{\rightarrow}{\rightarrow} \stackrel{0}{i} \stackrel{m}{\rightarrow} \rightrightarrows \stackrel{\infty}{i} \stackrel{\circ}{\rightarrow}$ & 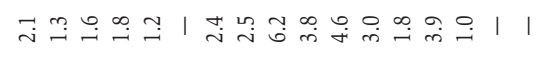 \\
\hline 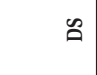 & 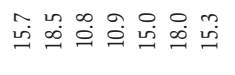 & 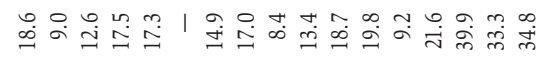 \\
\hline 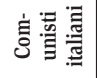 & 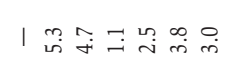 & 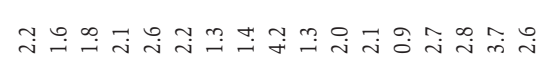 \\
\hline 容产 & 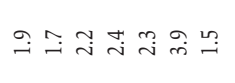 & 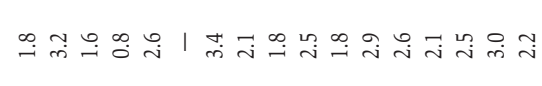 \\
\hline 焉事 & 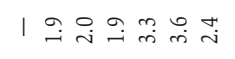 & 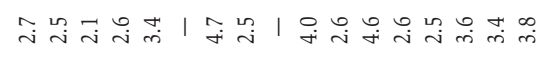 \\
\hline 离言总 & 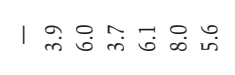 & 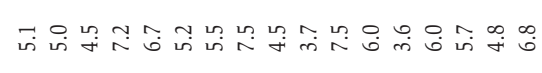 \\
\hline 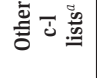 & $\check{\sigma} \stackrel{\infty}{\rightarrow} \stackrel{n}{\rightarrow} \stackrel{\infty}{0} \mid \stackrel{\circ}{\rightarrow} \stackrel{m}{\rightarrow}$ & | \\
\hline & 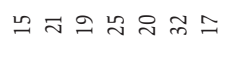 & 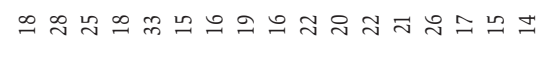 \\
\hline 完: & $n$ o n $\cos 9$ & $\exists a+n 0 \infty a \Rightarrow n=\exists$ in in \\
\hline 递 & 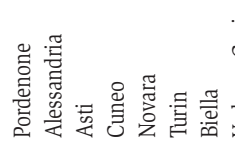 & 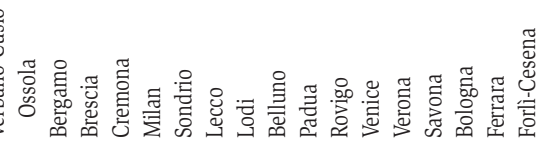 \\
\hline
\end{tabular}


응ㅇㅇㅇㅇㅇㅇㅇㅇㅇㅇㅇㅇㅇㅇㅇㅇㅇㅇㅇㅇㅇㅇㅇㅇㅇㅇㅇㅇㅇㅇㅇㅇㅇㅇㅇㅇㅇㅇㅇㅇㅇㅇ 음

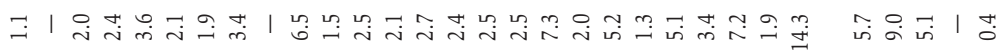

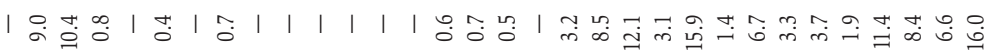

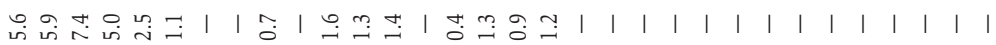
$\vec{n} \tilde{b}$ 范

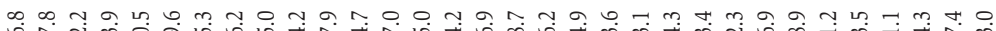
ப்

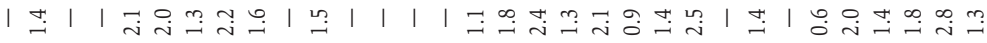

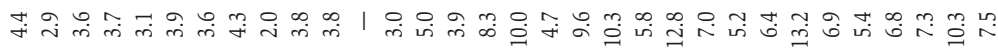

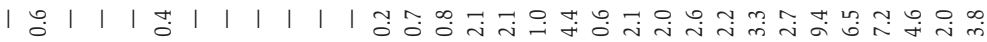

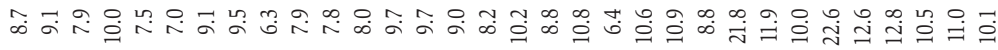

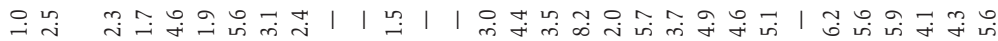
m

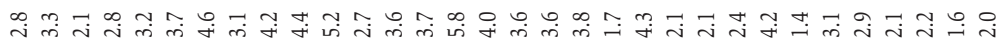
 㒸

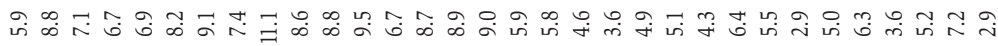

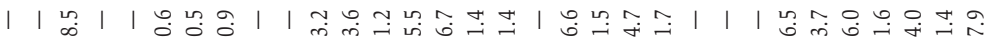

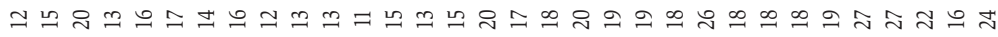

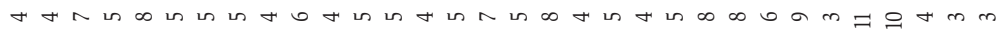

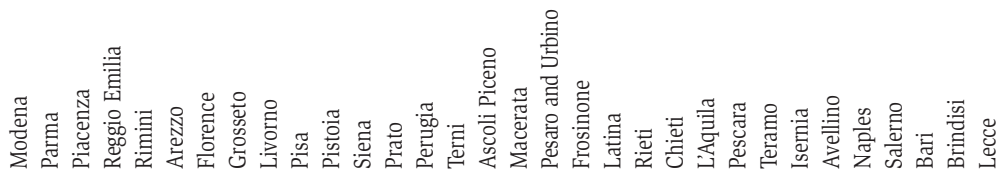




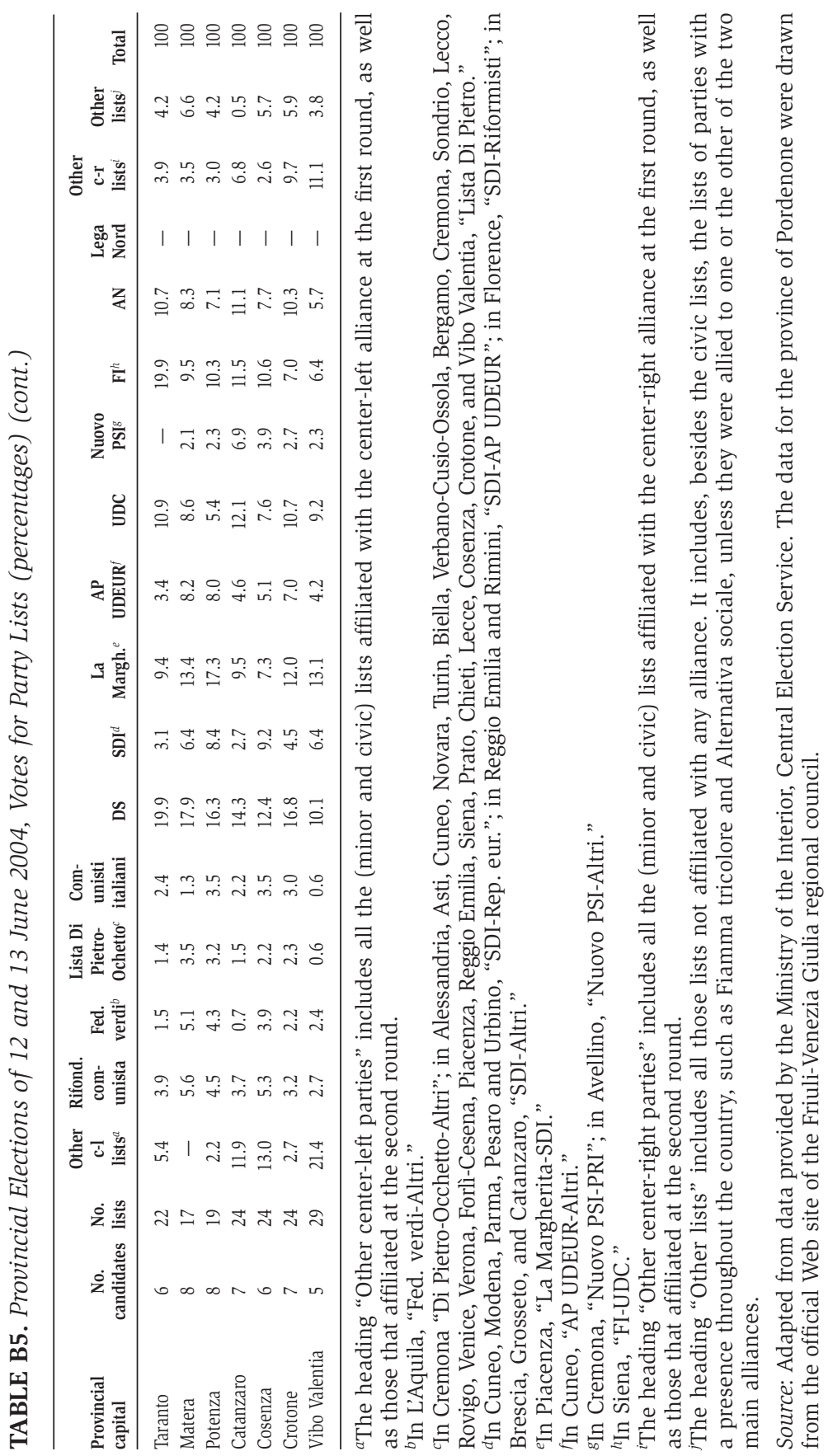




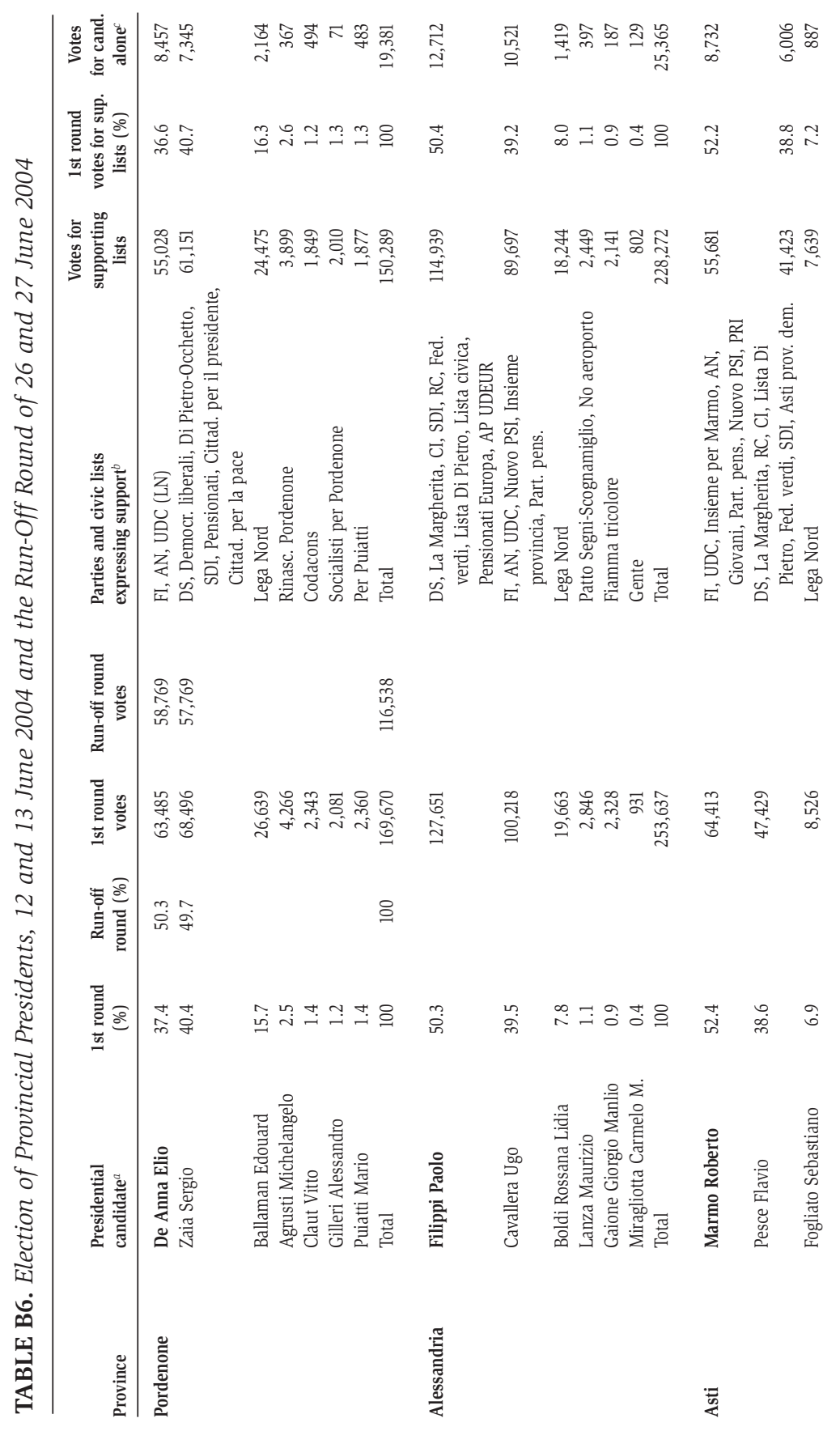




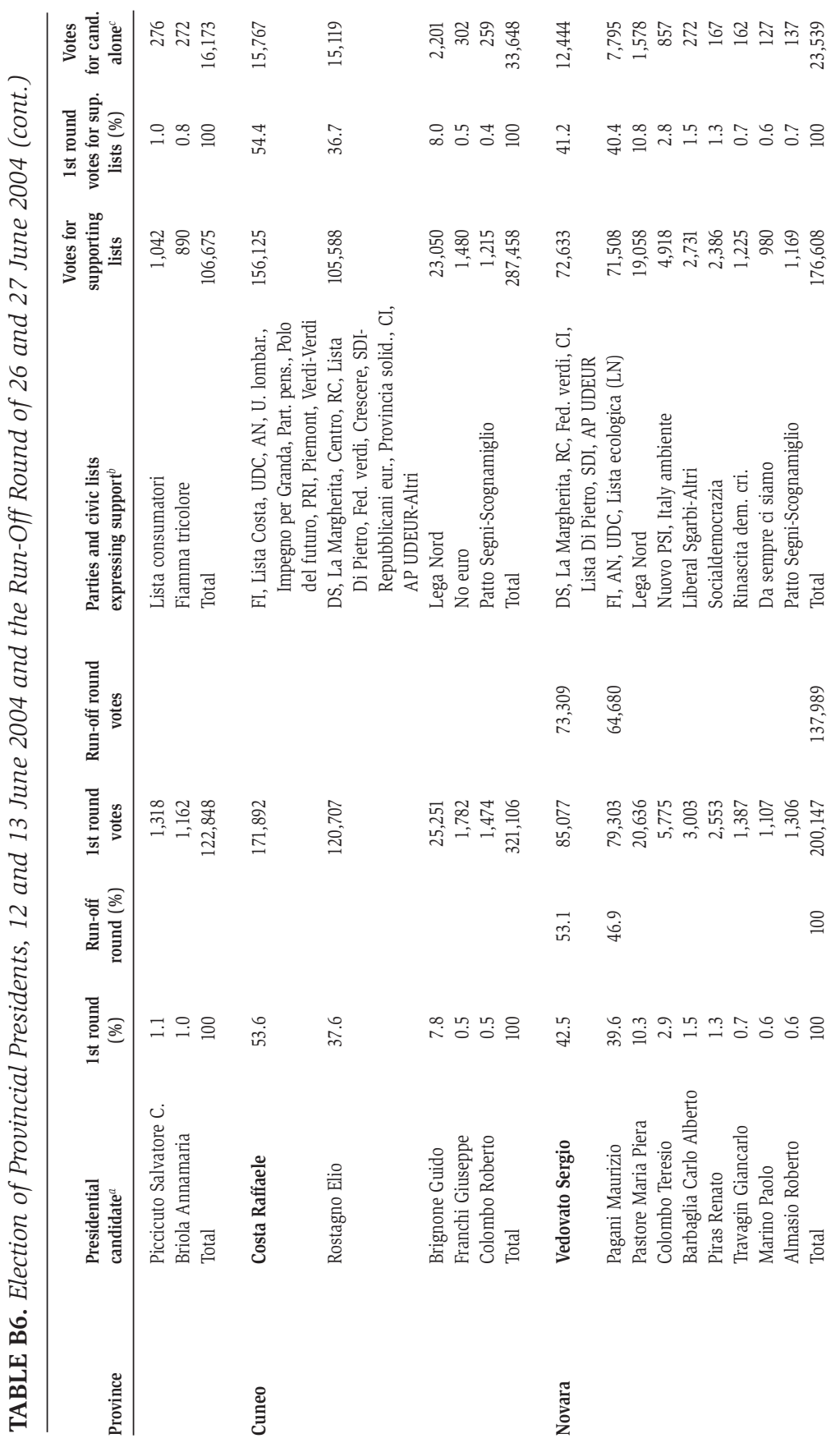




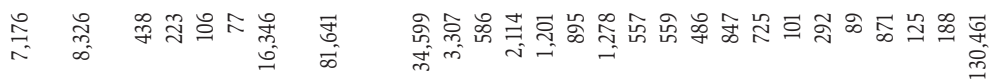

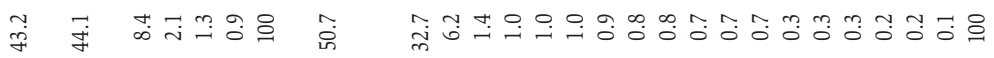

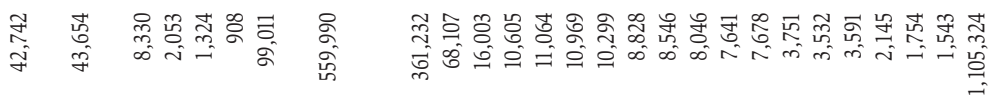
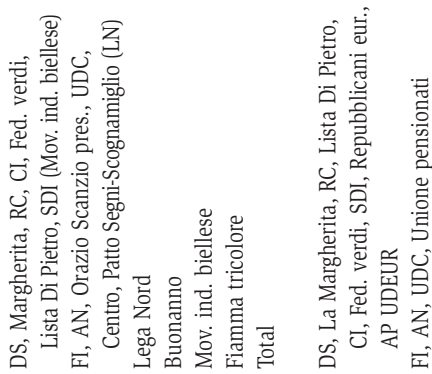

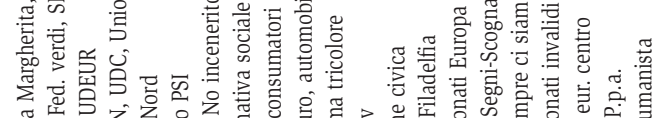

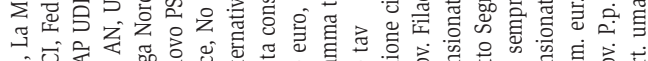

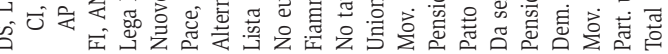

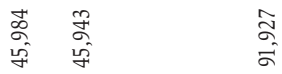

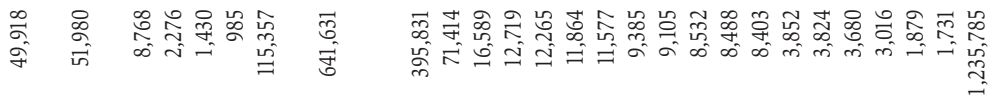

ஜำ ஜ

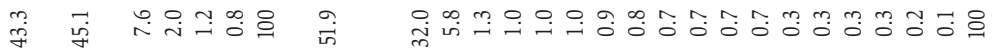

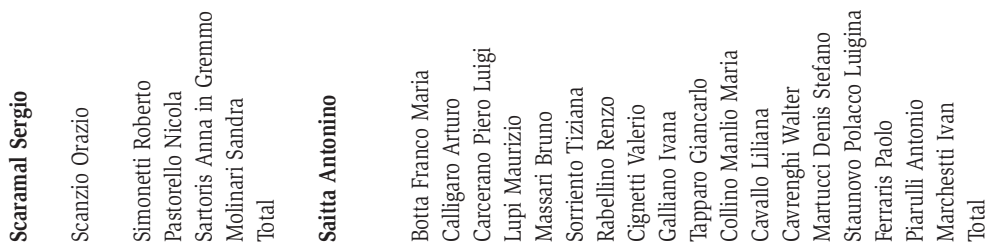




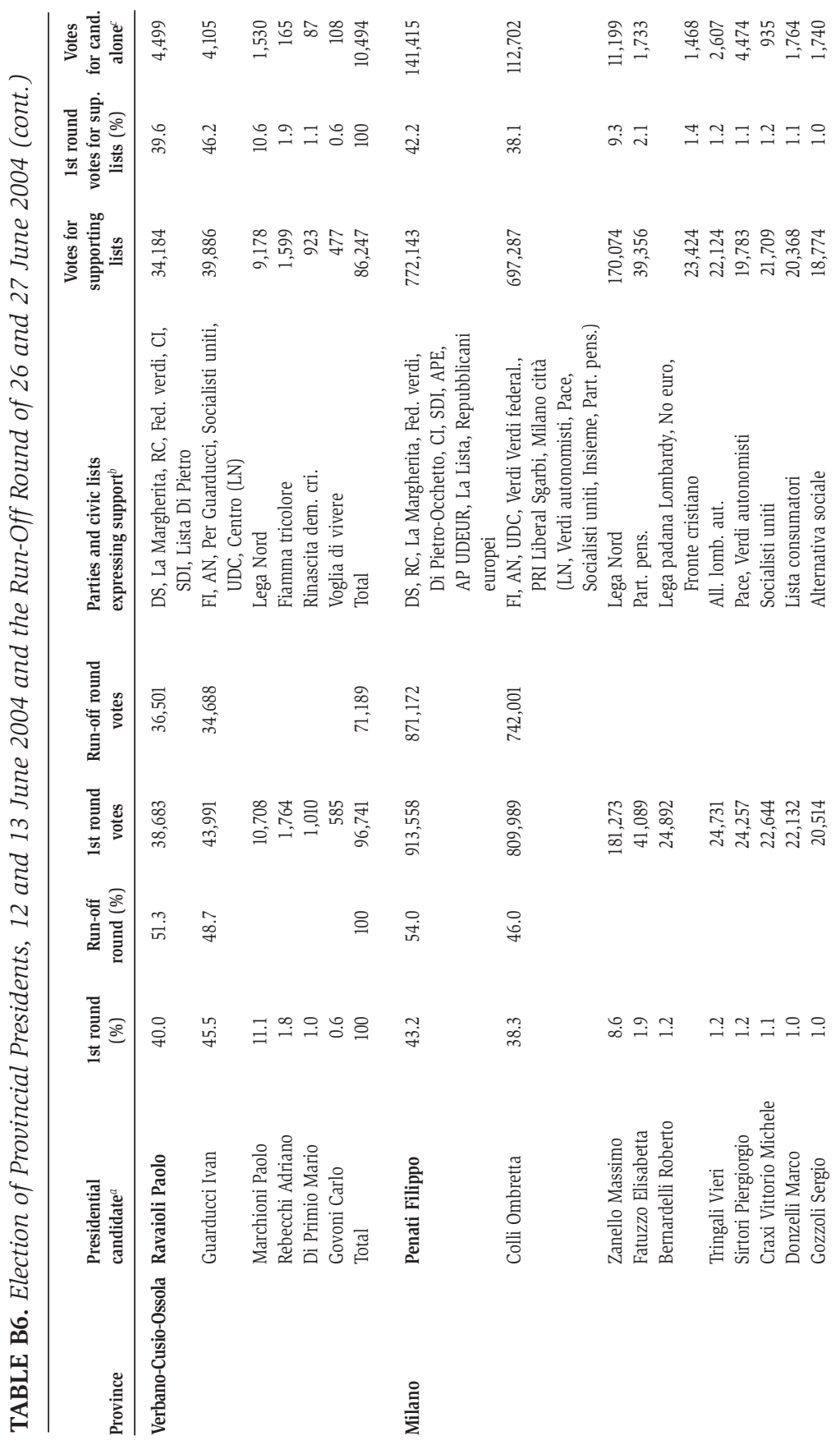




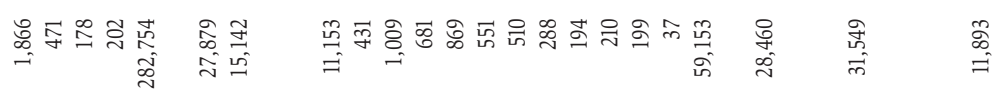

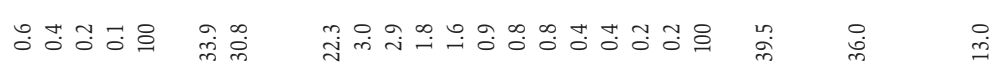

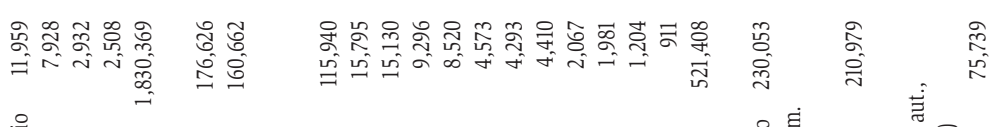

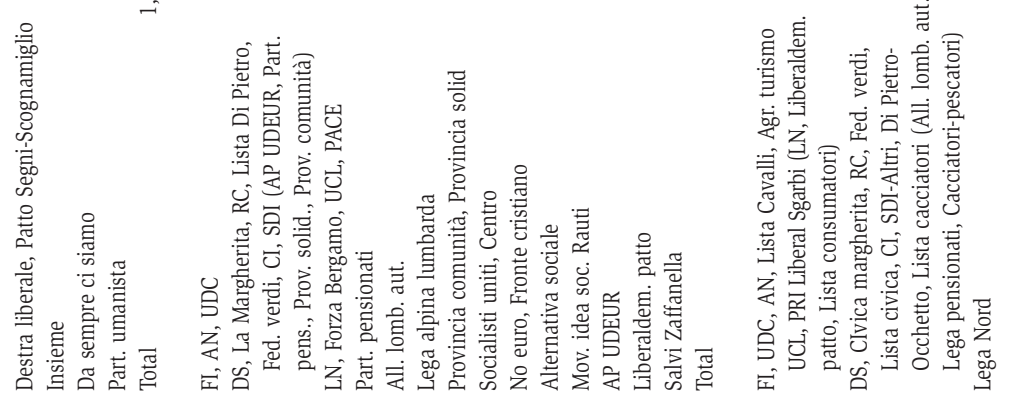

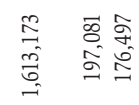

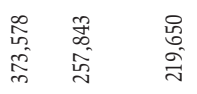

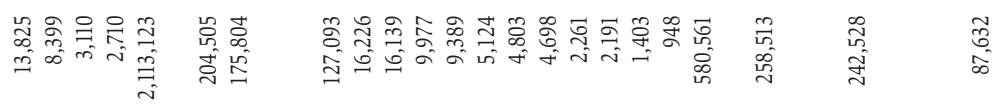

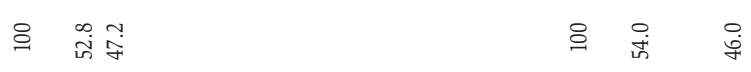

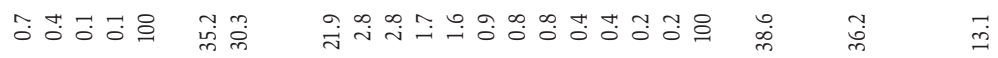

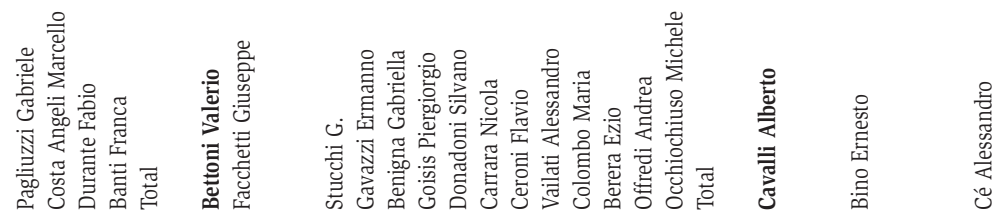




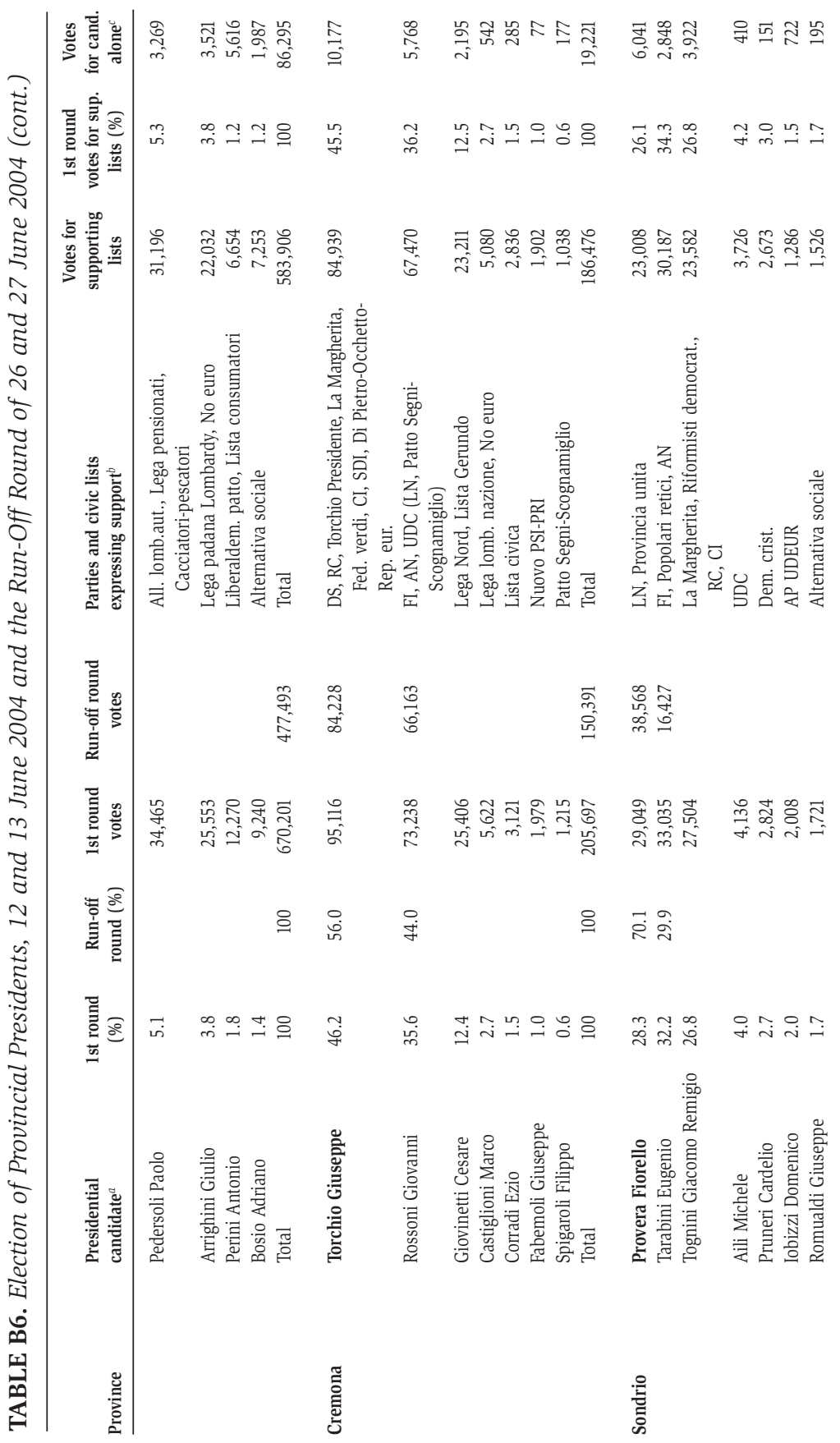




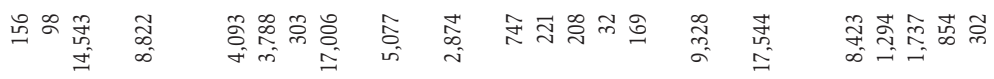

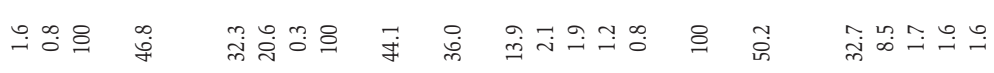

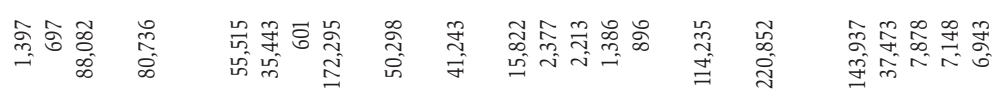

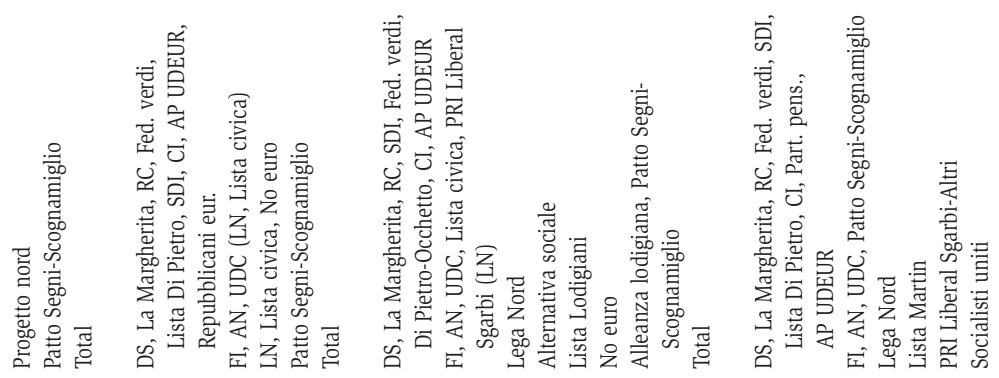

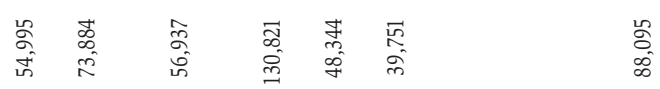

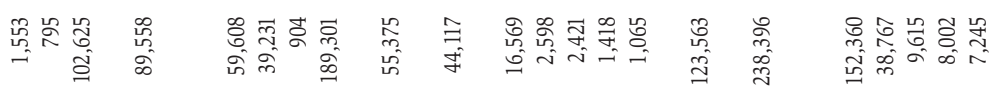

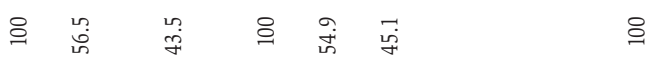

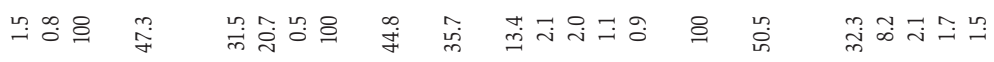

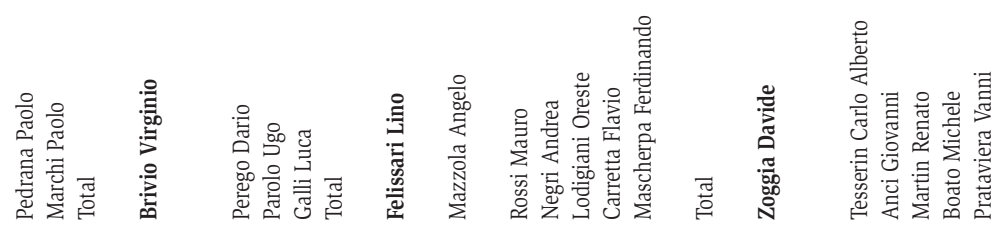




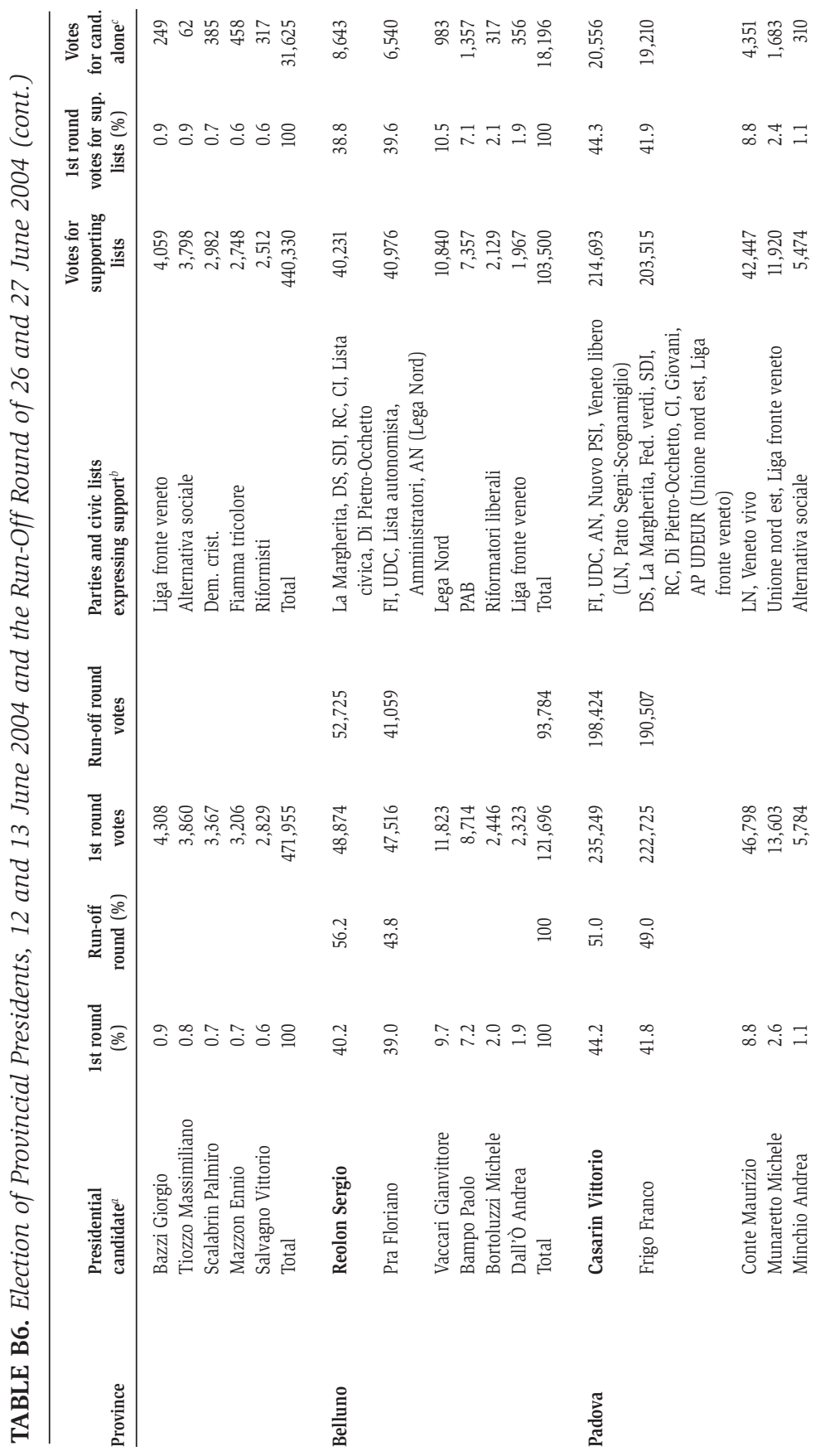




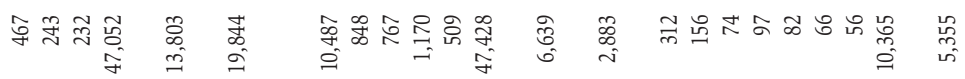

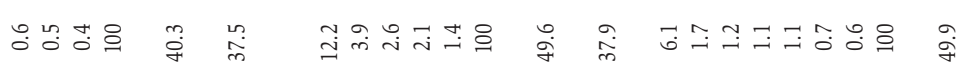

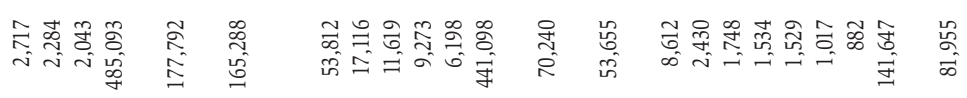

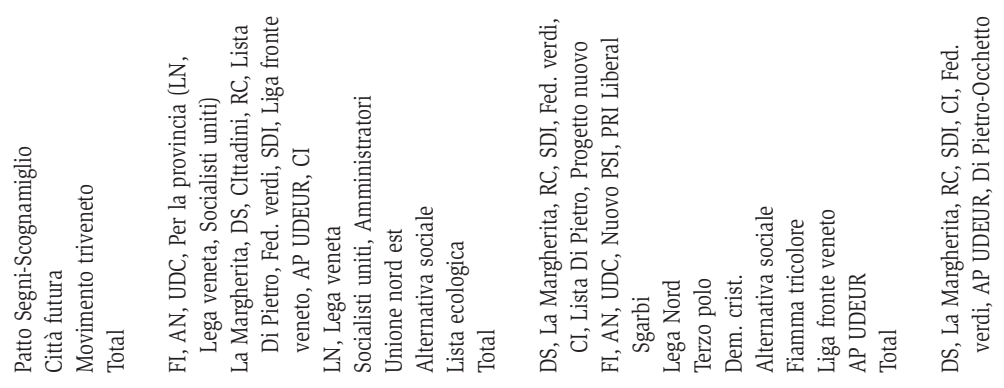

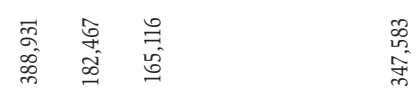

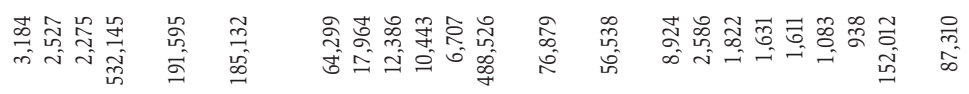

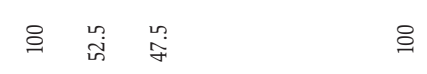

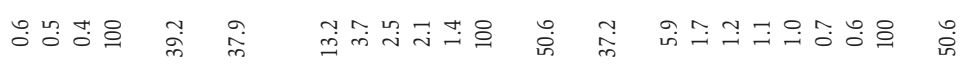

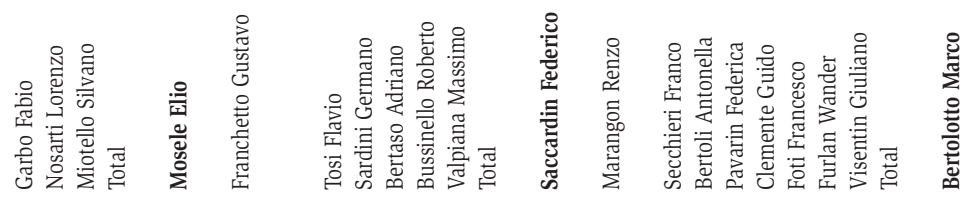




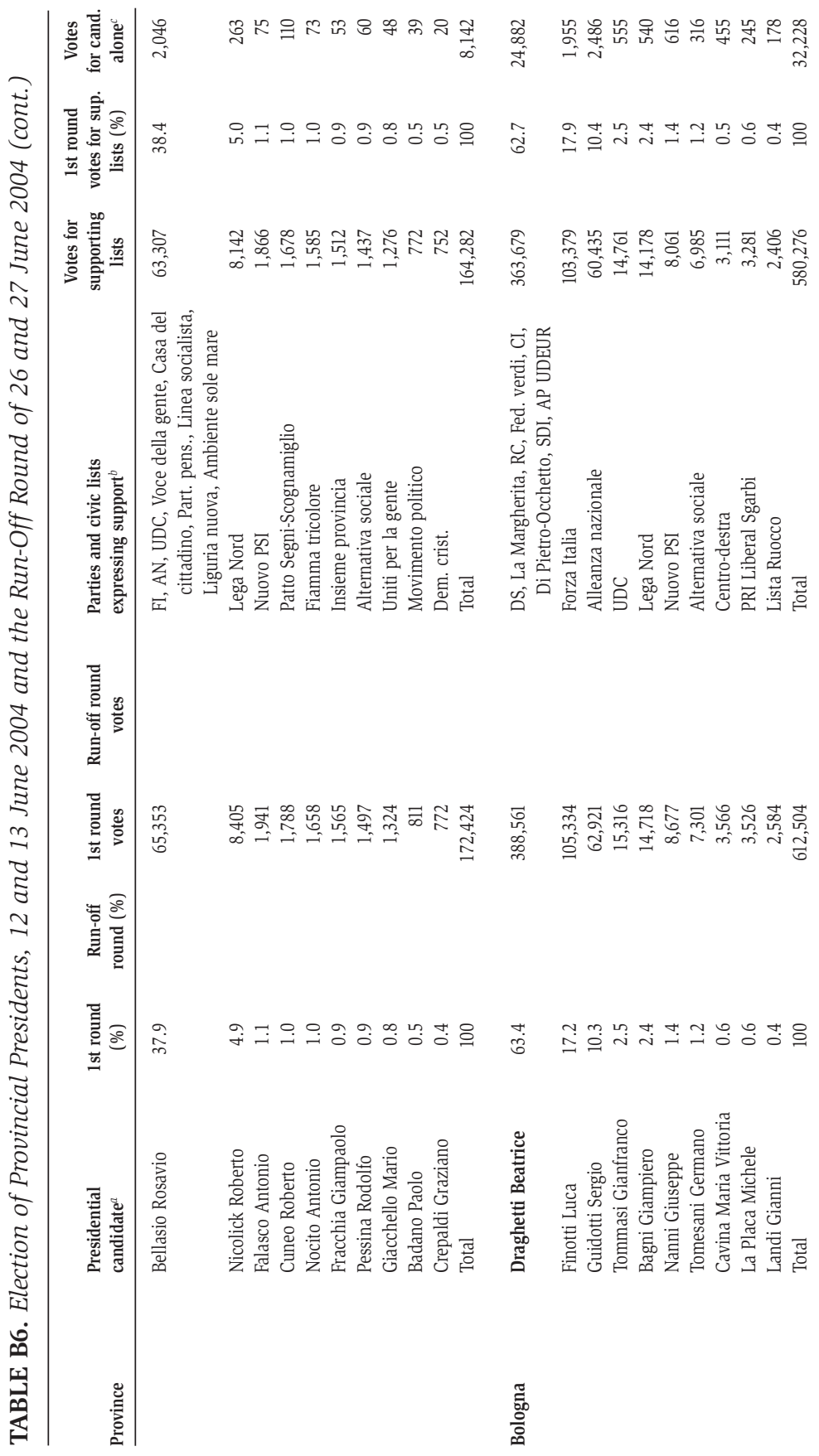




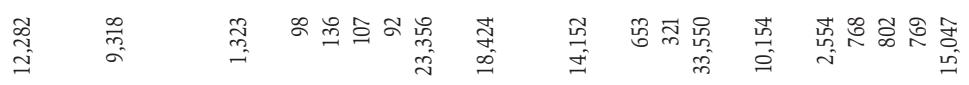

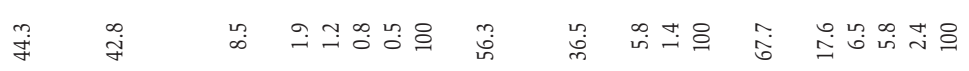

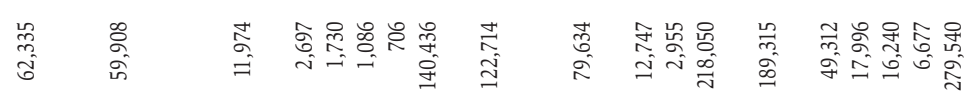

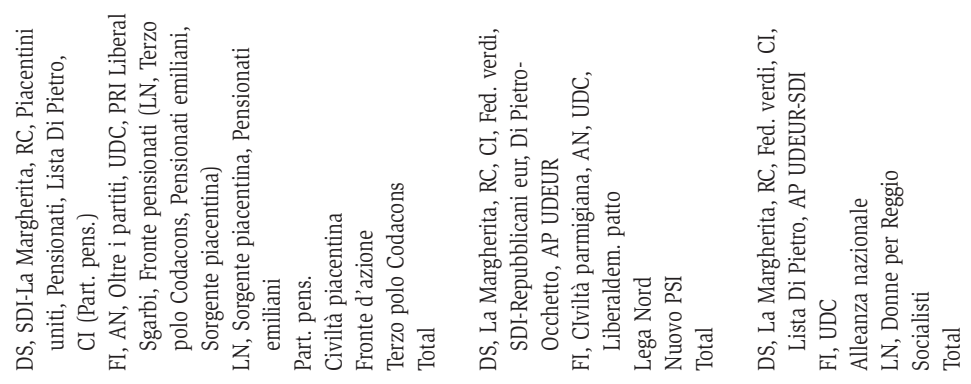

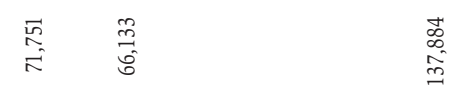

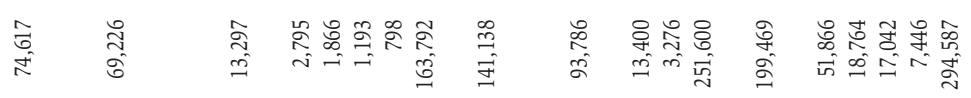

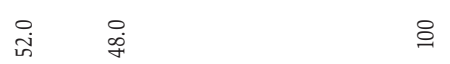

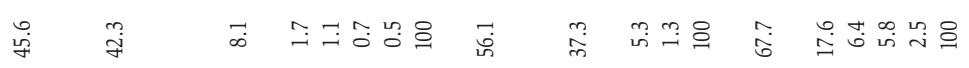

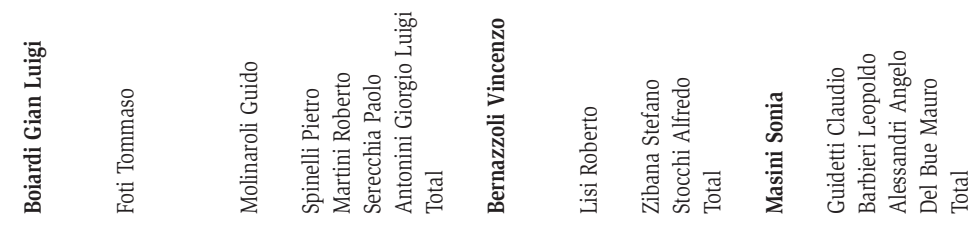




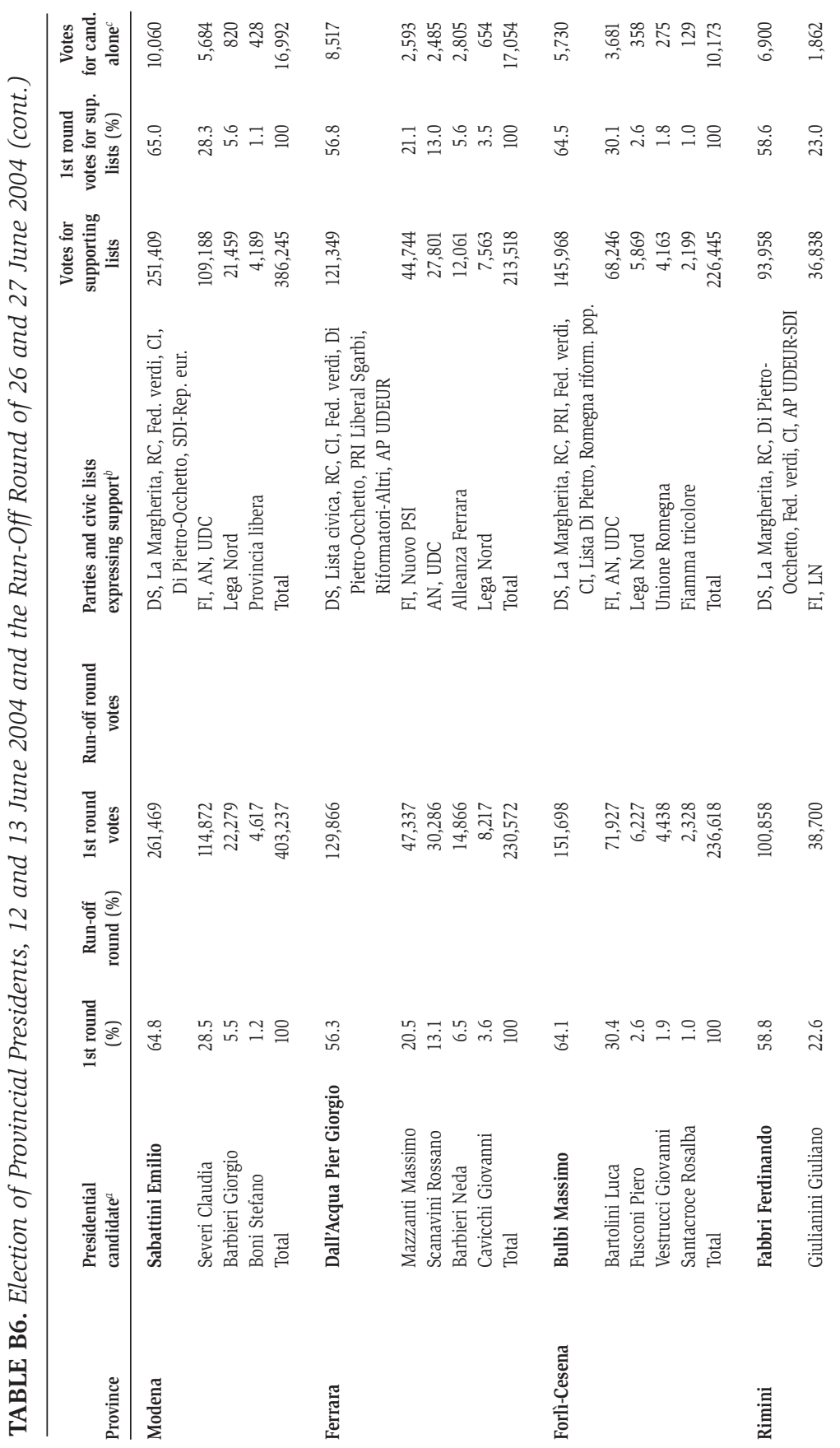




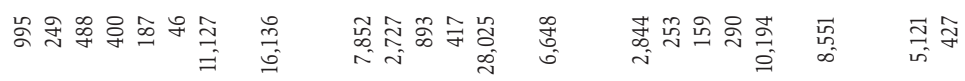

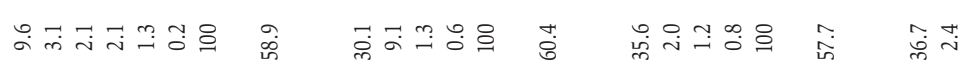

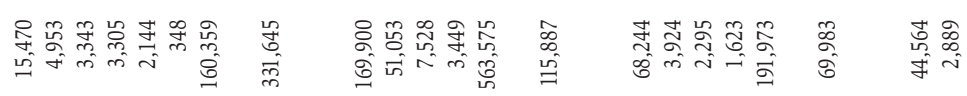

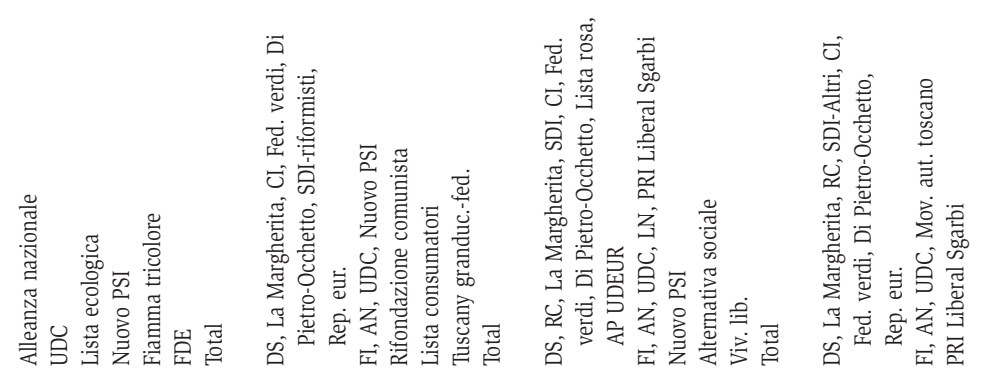

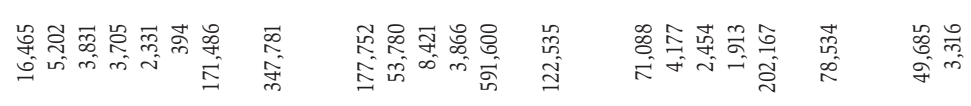

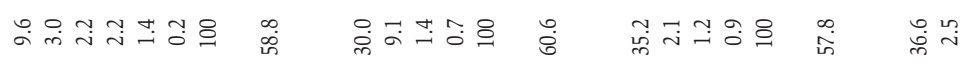

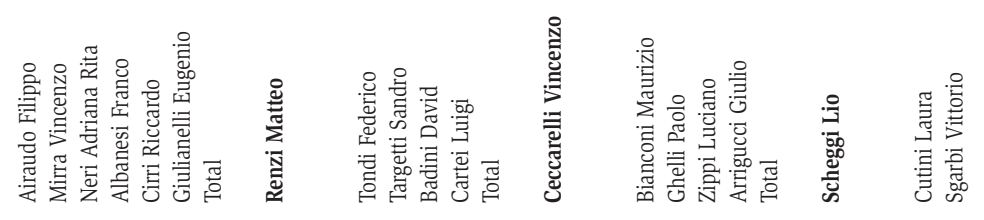

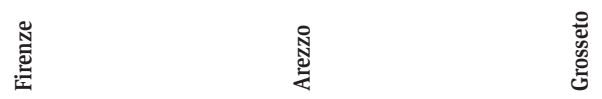




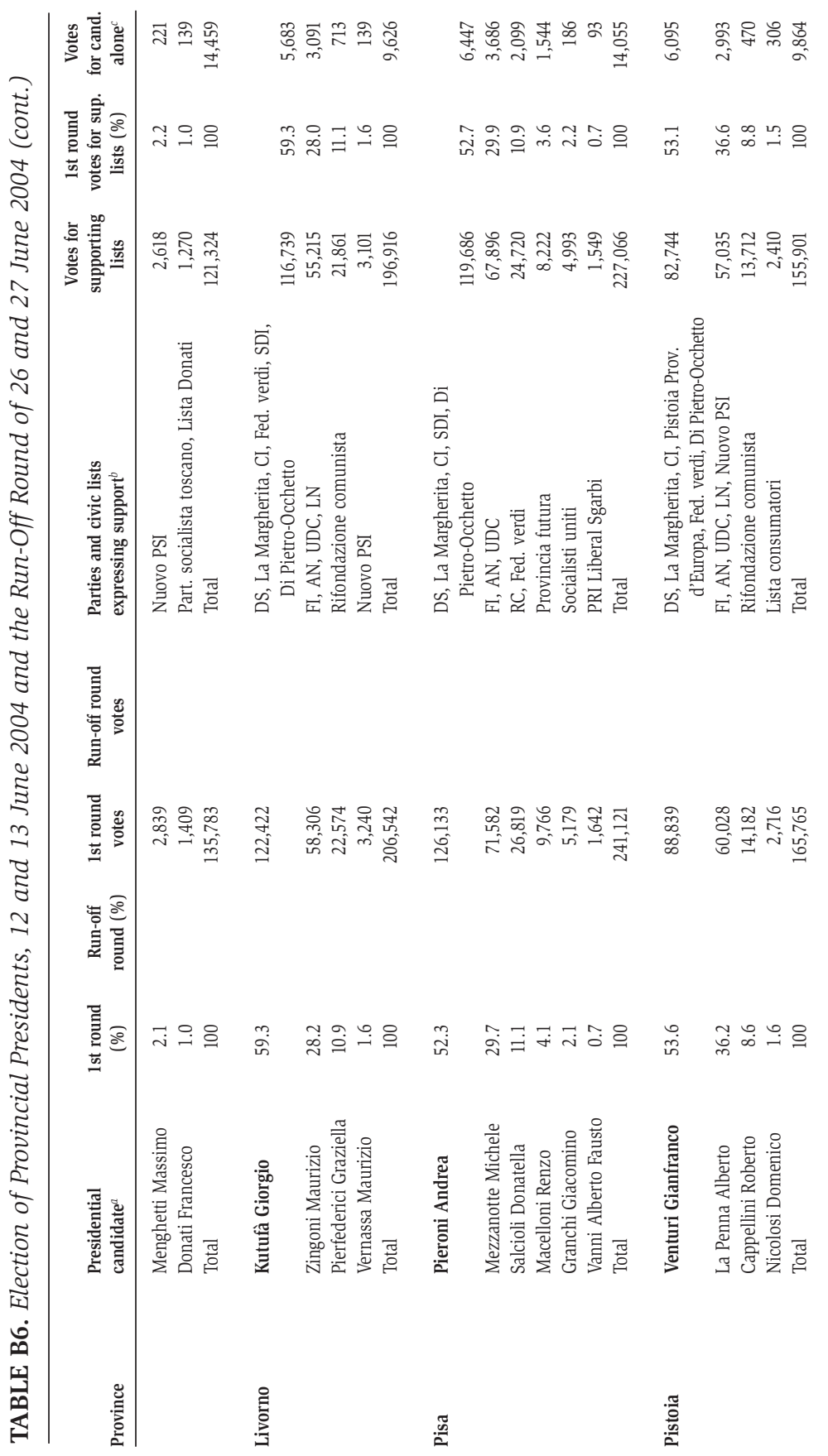




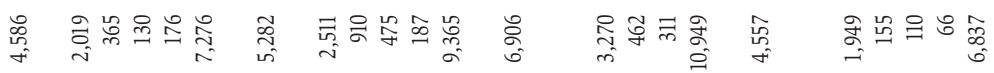

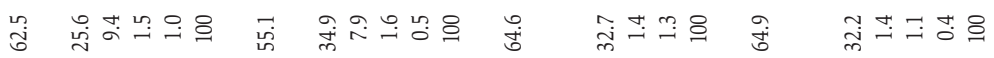

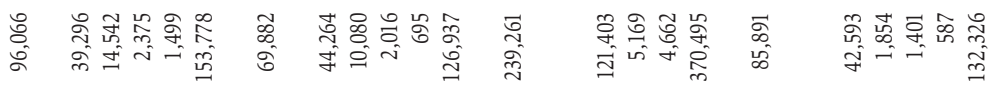

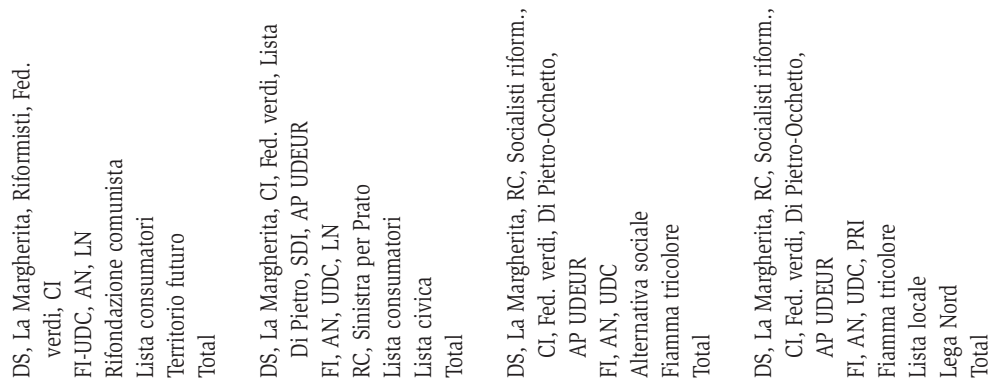

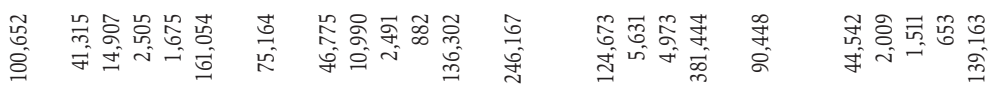

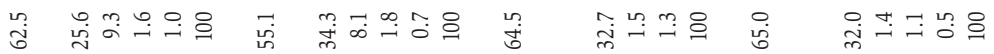

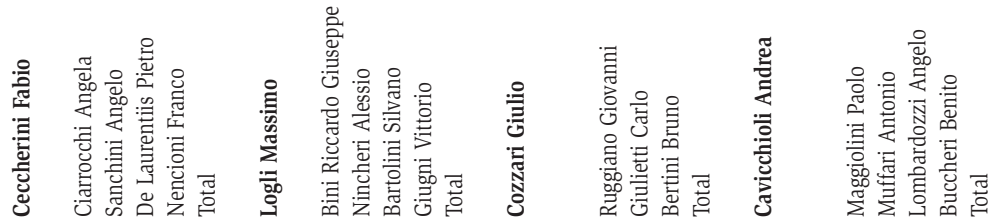

苛

总

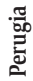

芫 


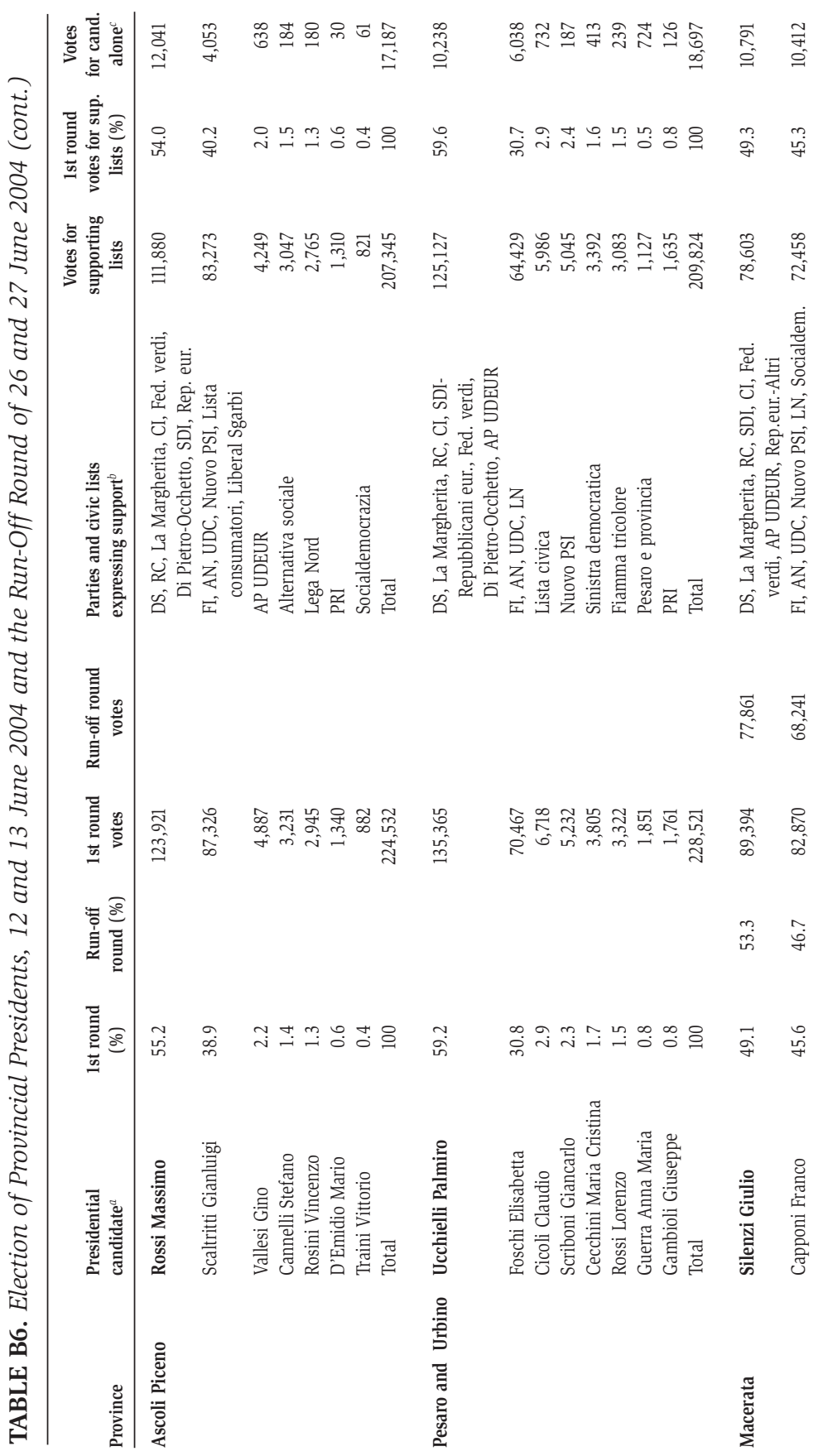




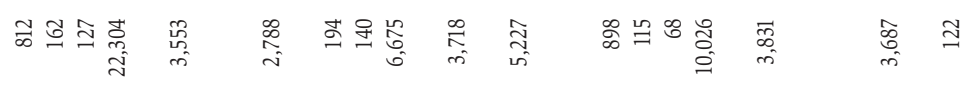

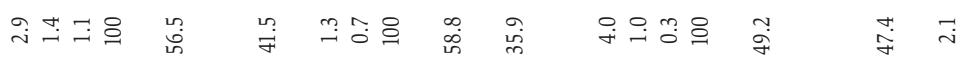

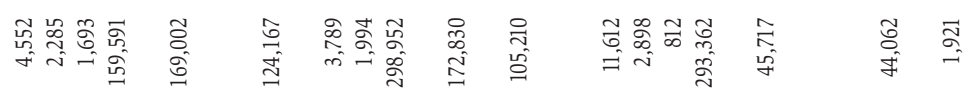

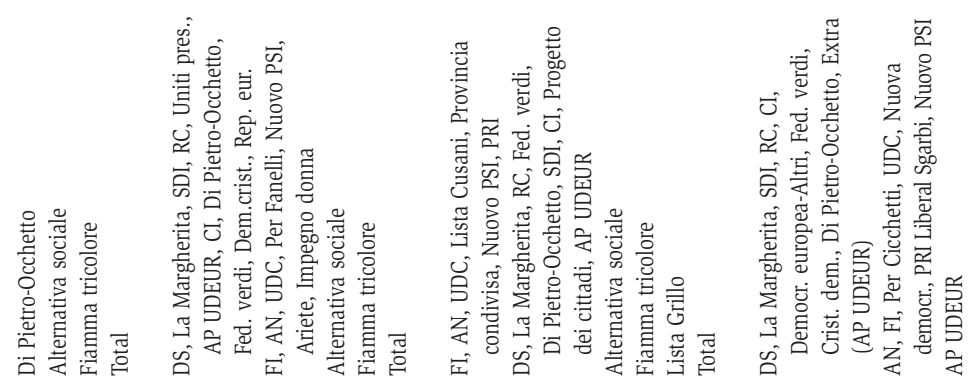

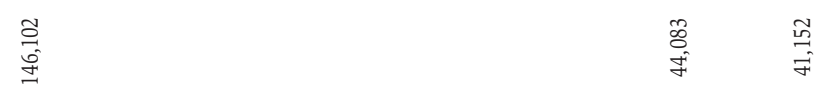

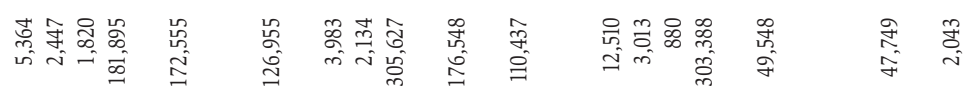

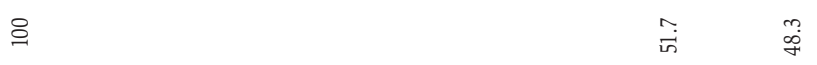

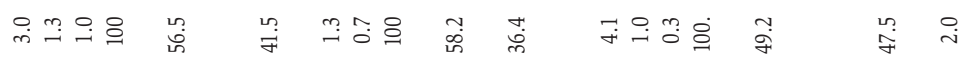

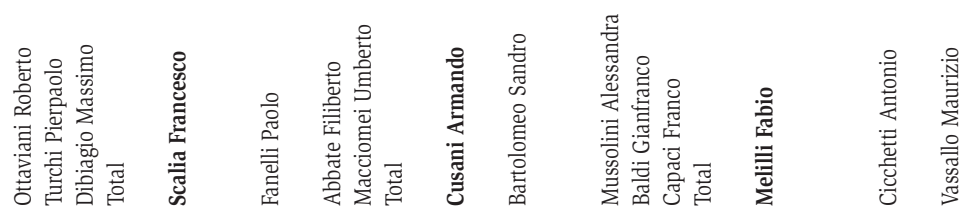

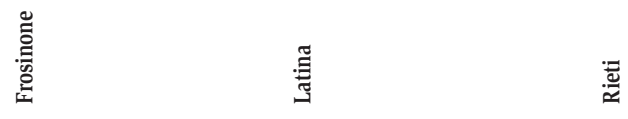




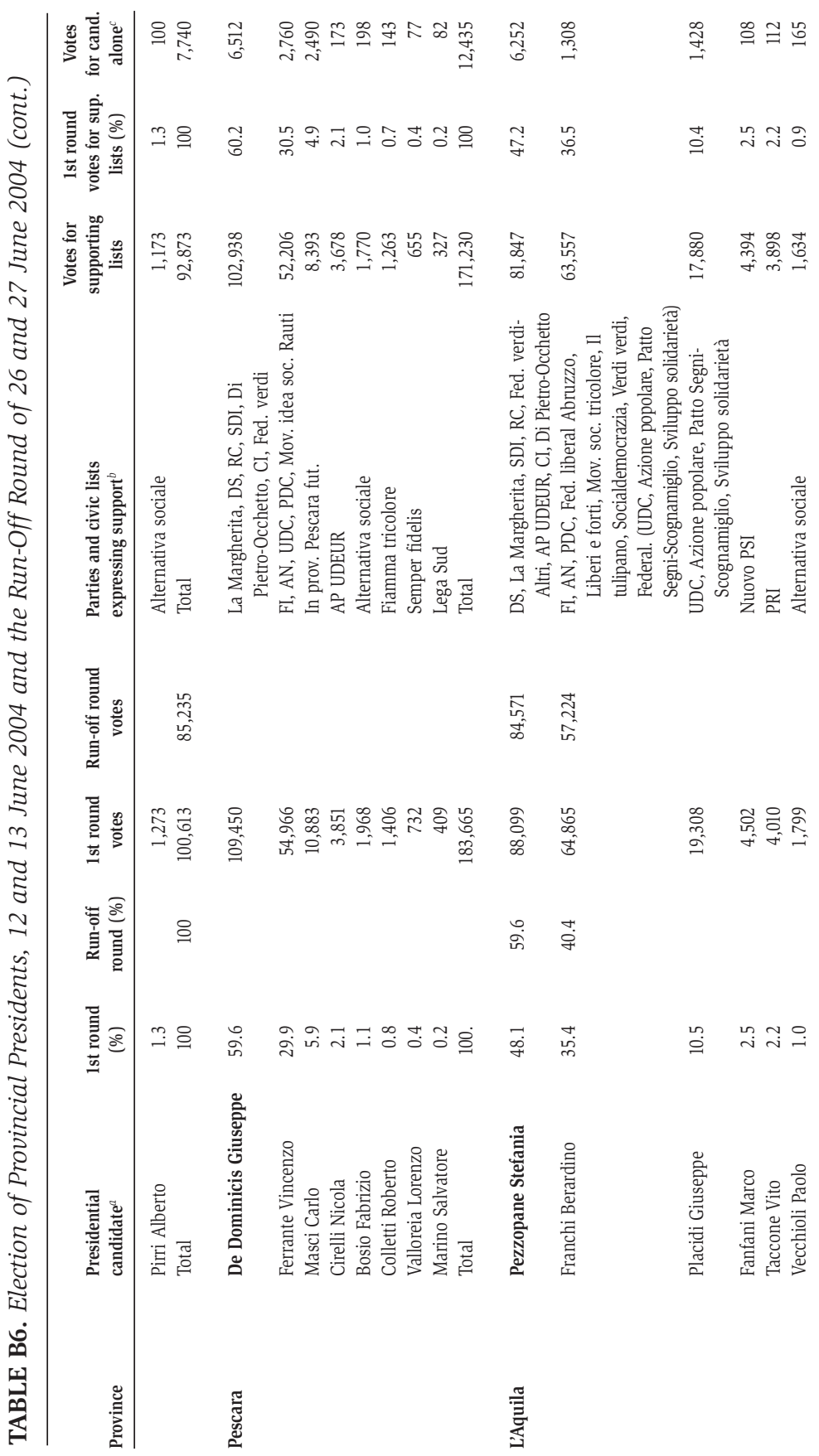




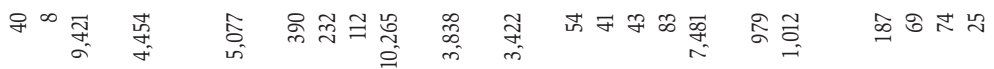

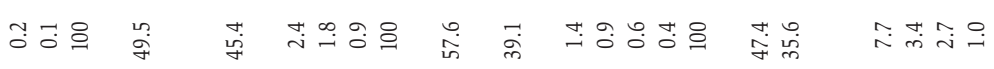

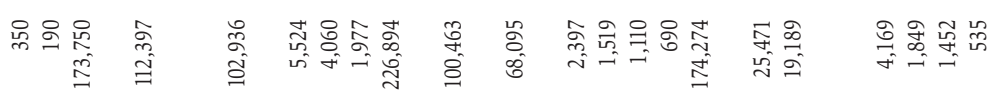

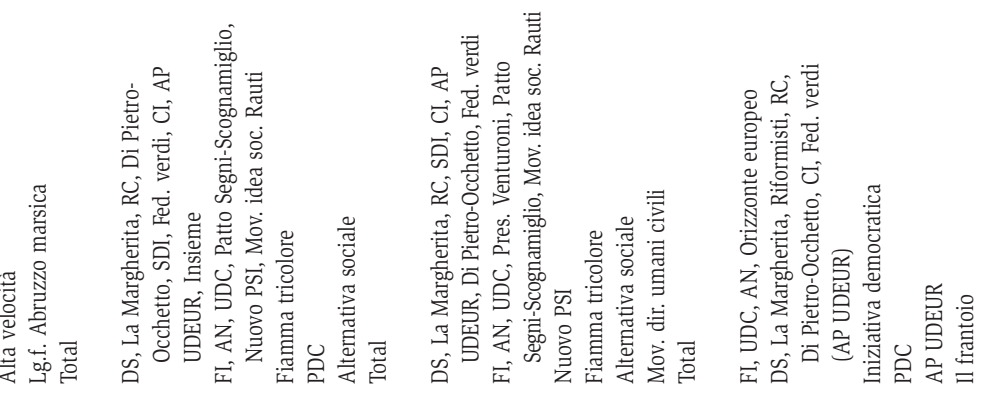

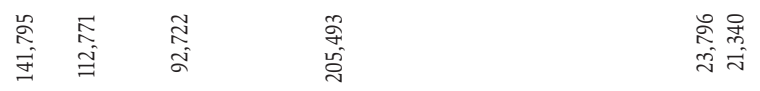

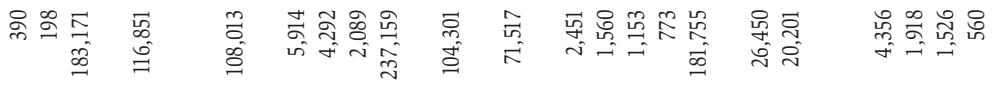

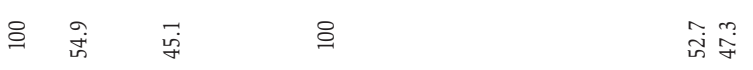

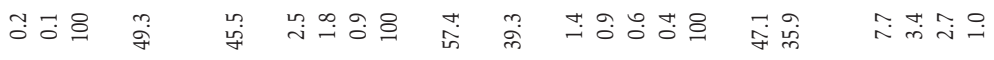

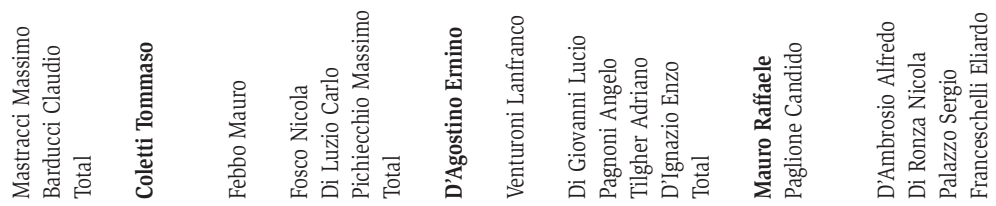




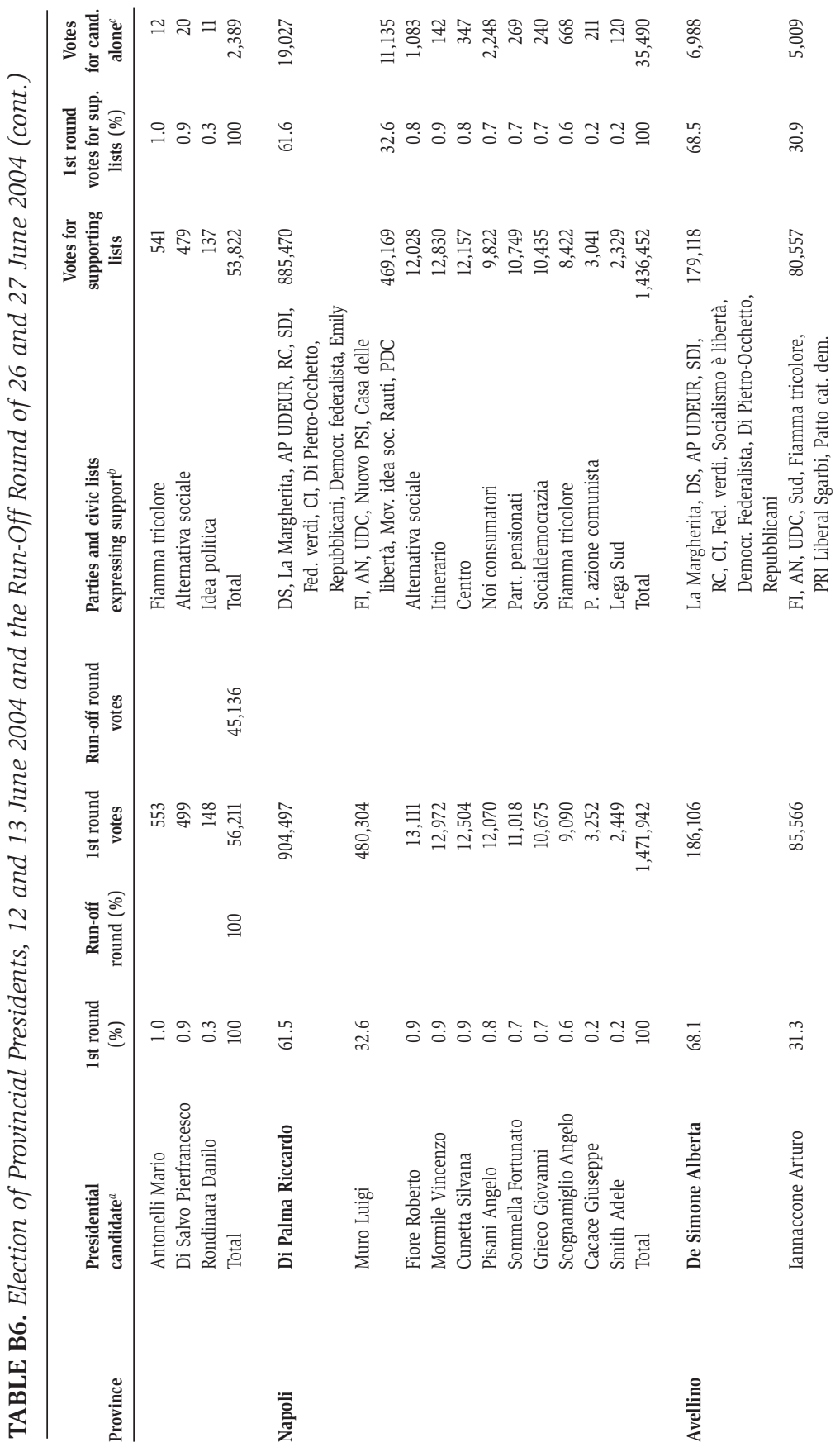


হ

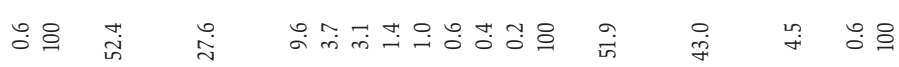

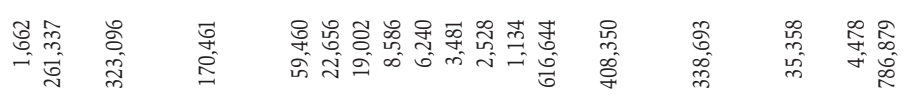

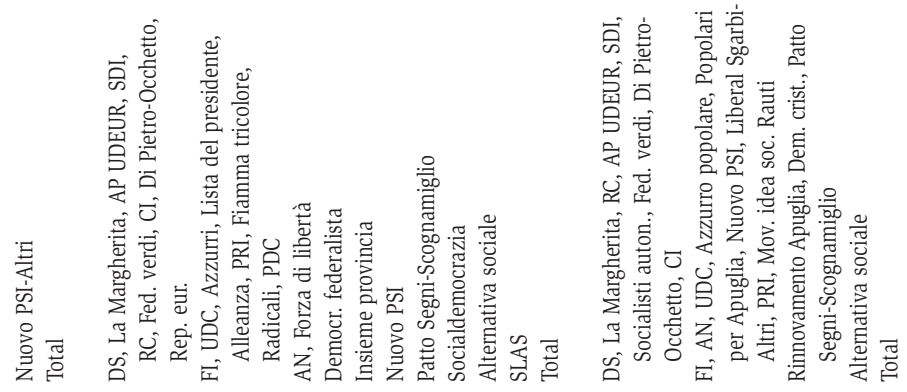

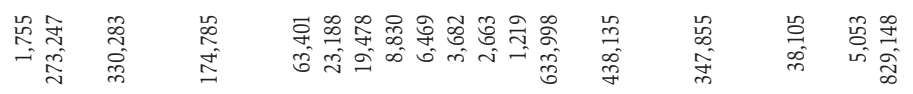

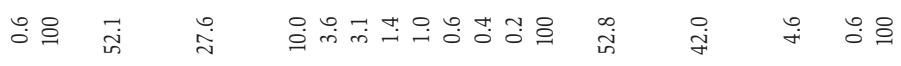

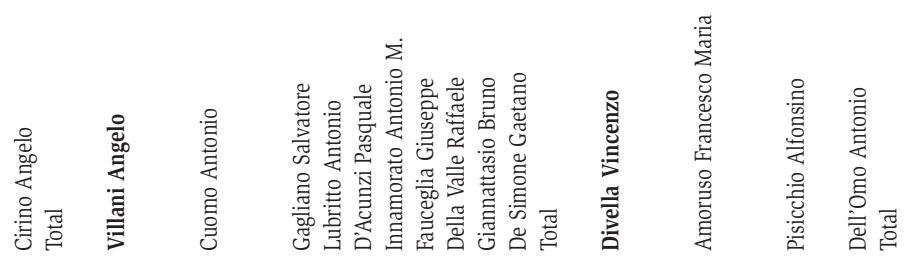

苞 


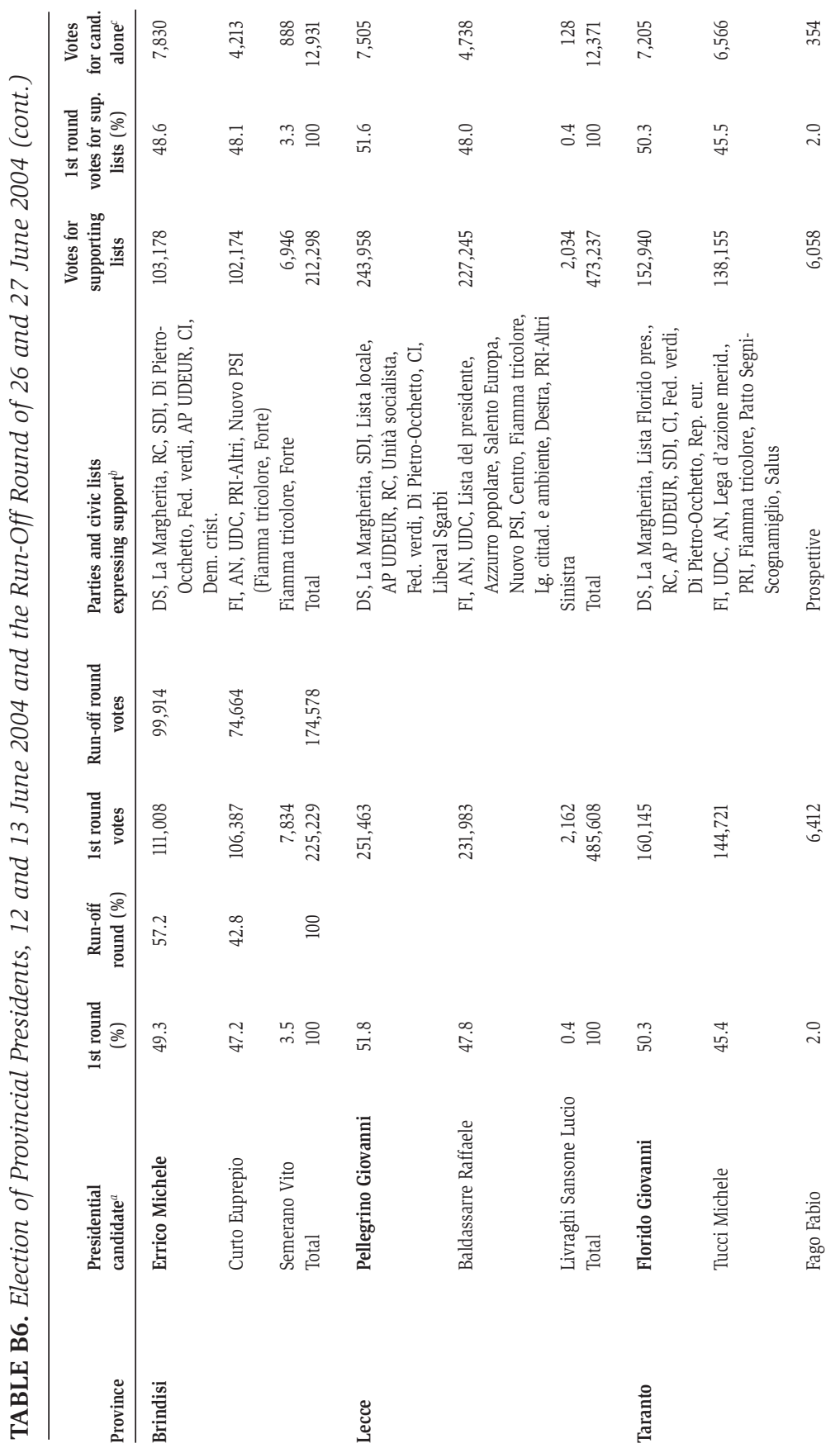




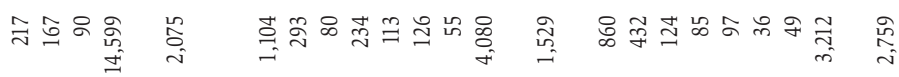

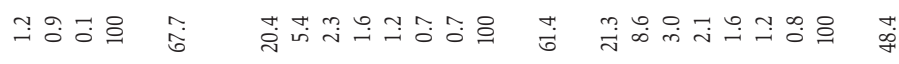

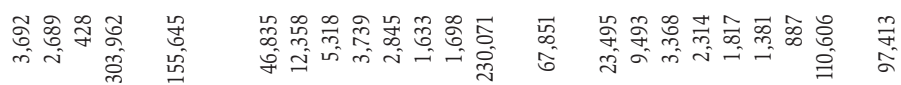

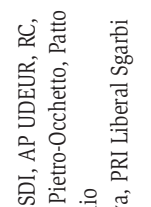

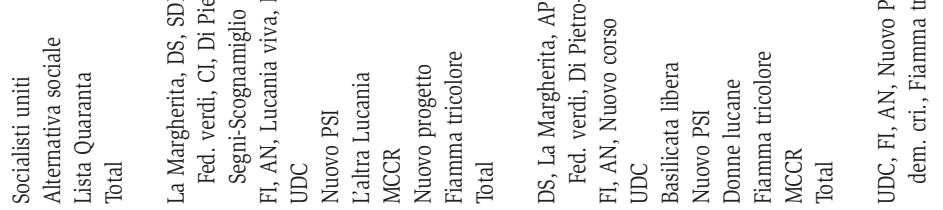

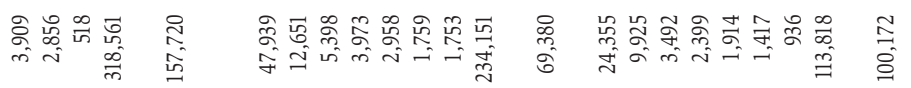

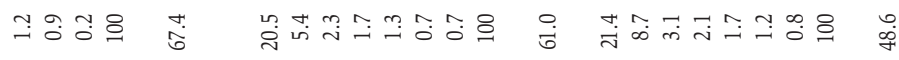

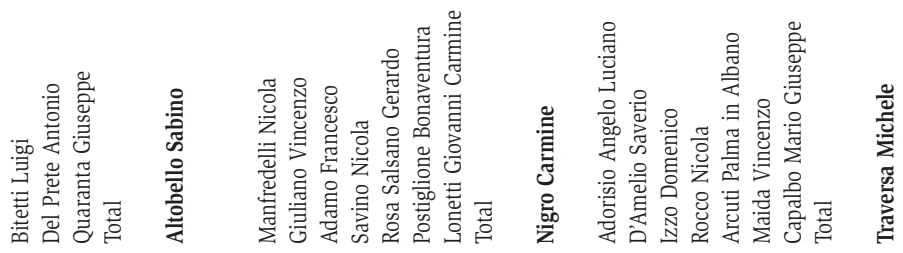




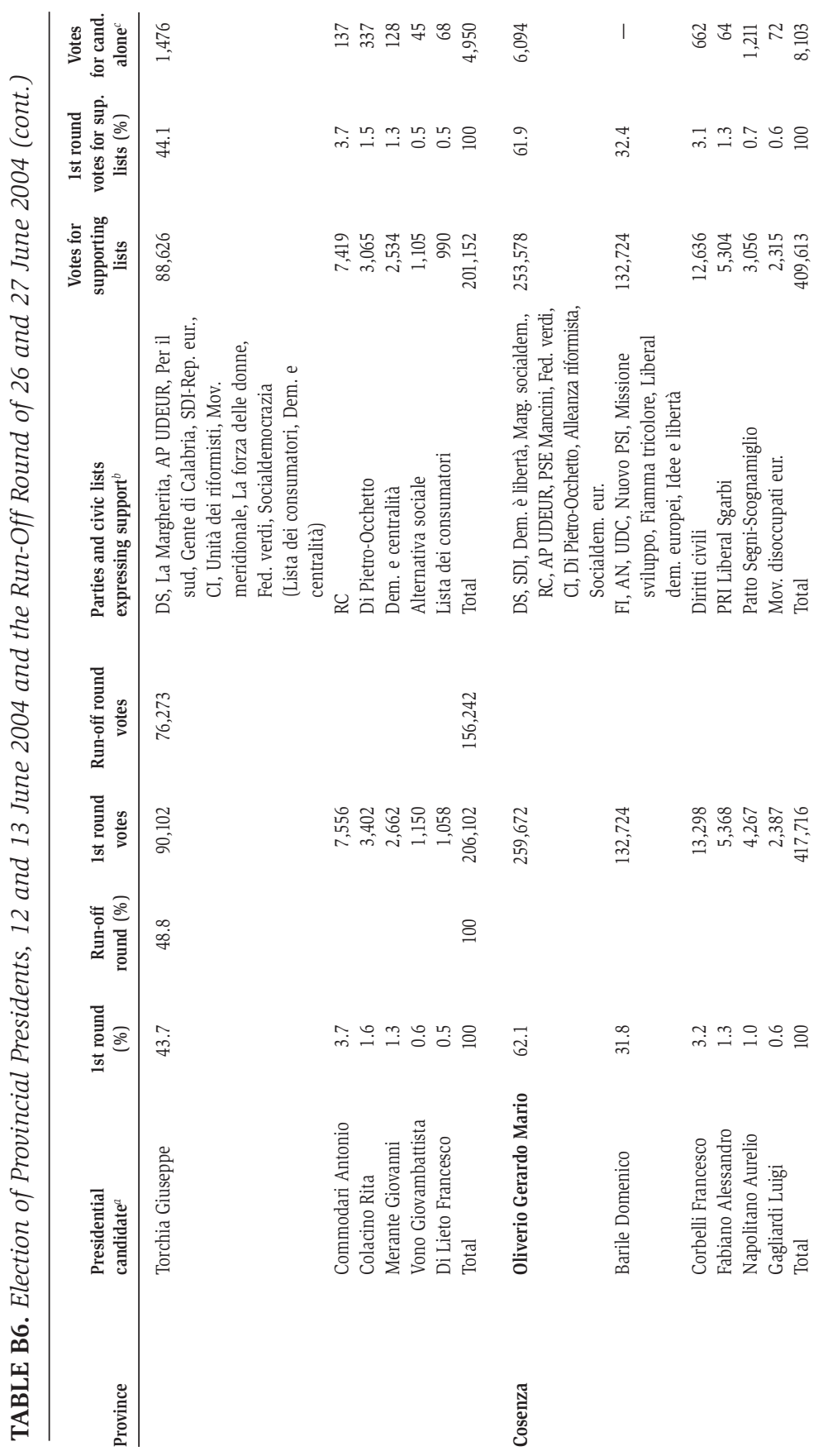




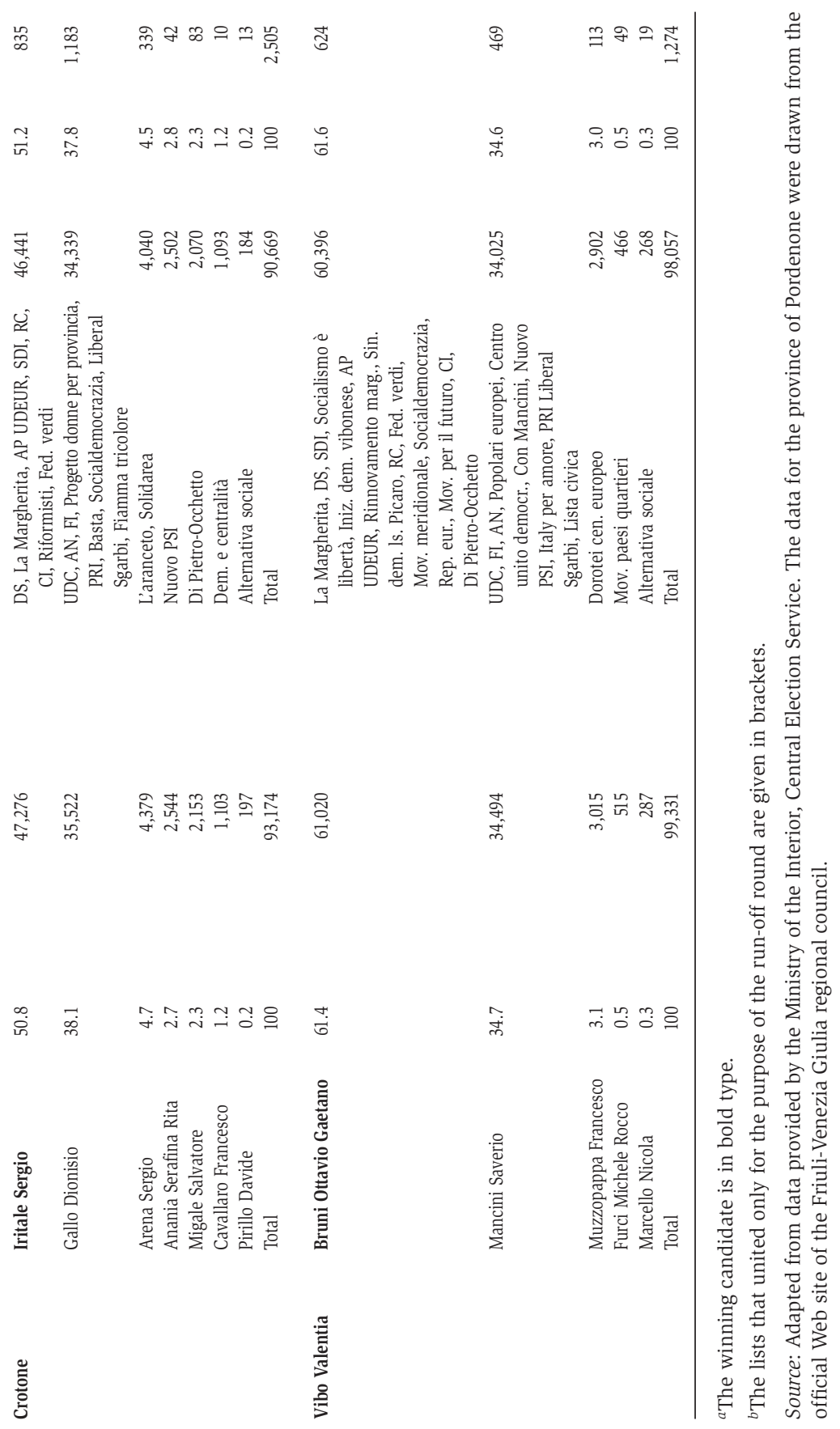


European Elections, 2004

TABLE B7a. Turnout at the European Parliament Elections of 12 and 13 June 2004

\begin{tabular}{lrrrrrrr}
\hline Region & \multicolumn{1}{c}{$\begin{array}{c}\text { \% Voting } \\
\text { (ontitled }\end{array}$} & \multicolumn{1}{c}{ to vote- } & \multicolumn{1}{c}{ Voters } & $\begin{array}{c}\text { torate) } \\
\text { Valid } \\
\text { votes }\end{array}$ & $\begin{array}{c}\text { \% Valid } \\
\text { votes (of } \\
\text { voters) }\end{array}$ & $\begin{array}{r}\text { Non- } \\
\text { valid } \\
\text { votes }\end{array}$ & $\begin{array}{c}\text { \% Blank } \\
\text { ballots } \\
\text { (of non- } \\
\text { valid votes) }\end{array}$ \\
\hline Valle d'Aosta & 101,972 & 63,156 & 61.9 & 56,3336 & 89.2 & 6,820 & 38.2 \\
Piedmont & $3,622,313$ & $2,731,406$ & 75.4 & $2,480,500$ & 90.8 & 250,906 & 51.6 \\
Lombardy & $7,570,425$ & $5,783,795$ & 76.4 & $5,423,989$ & 93.8 & 359,806 & 47.3 \\
Trentino-Alto A. & 769,560 & 530,776 & 69.0 & 500,483 & 94.3 & 30,293 & 40.2 \\
Veneto & $3,837,535$ & $2,950,190$ & 76.9 & $2,755,656$ & 93.4 & 194,534 & 49.1 \\
Friuli-Venezia G. & $1,053,835$ & 735,673 & 69.8 & 690,700 & 93.9 & 44,973 & 43.5 \\
Liguria & $1,392,234$ & $1,006,814$ & 72.3 & 947,345 & 94.1 & 59,469 & 43.2 \\
Emilia-Romagna & $3,406,968$ & $2,769,680$ & 81.3 & $2,624,404$ & 94.8 & 145,276 & 56.1 \\
Tuscany & $2,996,164$ & $2,339,099$ & 78.1 & $2,197,756$ & 94.0 & 141,343 & 52.1 \\
Umbria & 701,605 & 564,611 & 80.5 & 516,809 & 91.5 & 47,802 & 61.0 \\
Marches & $1,261,769$ & 981,918 & 77.8 & 894,567 & 91.1 & 87,351 & 59.5 \\
Lazio & $4,543,302$ & $3,258,673$ & 71.7 & $3,053,560$ & 93.7 & 205,113 & 39.2 \\
Abruzzo & $1,151,495$ & 859,324 & 74.6 & 749,427 & 87.2 & 109,897 & 60.8 \\
Molise & 308,278 & 210,161 & 68.2 & 179,104 & 85.2 & 31,057 & 60.1 \\
Campania & $4,733,347$ & $3,237,873$ & 68.4 & $2,847,133$ & 87.9 & 390,740 & 58.7 \\
Apuglia & $3,369,113$ & $2,425,666$ & 72.0 & $2,082,379$ & 85.8 & 343,287 & 54.2 \\
Basilicata & 526,568 & 392,030 & 74.5 & 320,436 & 81.7 & 71,594 & 57.4 \\
Calabria & $1,751,433$ & $1,169,381$ & 66.8 & $1,004,285$ & 85.9 & 165,096 & 57.3 \\
Sicily & $4,223,420$ & $2,556,925$ & 60.5 & $2,260,382$ & 88.4 & 296,543 & 36.3 \\
Sardinia & $1,384,309$ & $1,031,228$ & 74.5 & 890,973 & 86.4 & 140,255 & 49.4 \\
\hline
\end{tabular}

Source: Adapted from data provided by the Ministry of the Interior, Central Election Service. 


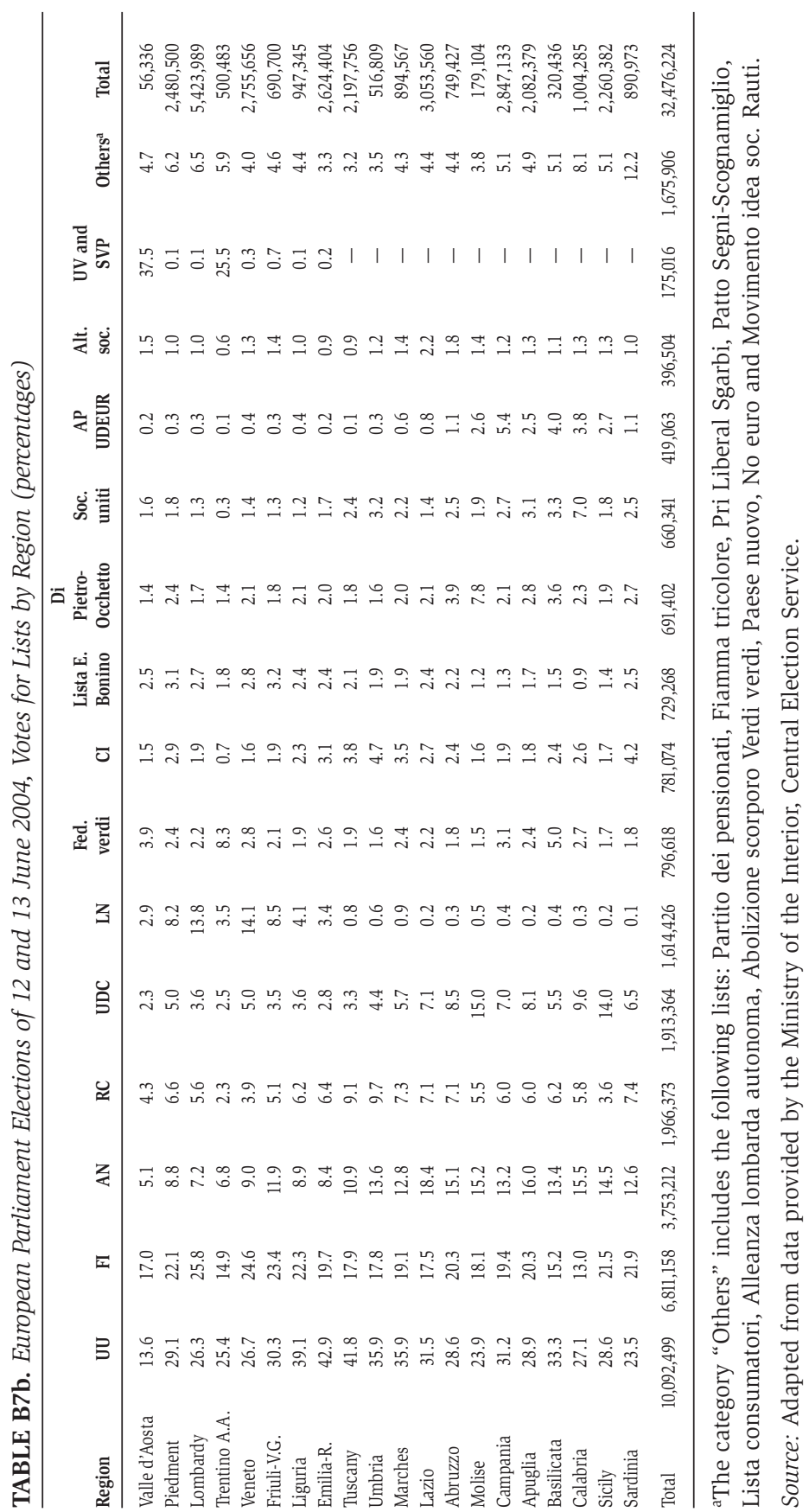


$\underline{\text { Regional Elections, 2004-Special Statute }}$

TABLE B8. Sardinian Regional Elections of 12 and 13 June 2004, Votes Cast for District Lists

\begin{tabular}{lr}
\hline Electorate & $1,449,052$ \\
Voters & $1,031,869$ \\
\% Voting (of electorate) & 71.2 \\
Valid votes & 857,303 \\
\% Valid votes (of voters) & 83.1 \\
Non-valid votes & 174,566 \\
\% Non-valid votes (of voters) & 16.9 \\
Votes cast for regional lists only & 115,478 \\
$\%$ (of voters) & 11.2 \\
\hline
\end{tabular}

Source: Adapted from data available through the official Web site of the Sardinian regional council: http//www.sitos.regione.sardegna.it/Elezioni-2004/index_2004.html.

TABLE B9. Sardinian Regional Elections of 12 and 13 June 2004, Valid Votes for District Lists

\begin{tabular}{lcc}
\hline District lists & Valid votes & \% Valid votes \\
\hline Forza Italia & 128,563 & 15.0 \\
Democratici di sinistra & 112,757 & 13.2 \\
La Margherita & 92,526 & 10.8 \\
UDC & 88,179 & 10.3 \\
Progetto Sardegna & 66,690 & 7.8 \\
Alleanza nazionale & 63,001 & 7.3 \\
Riformatori & 50,953 & 5.9 \\
Fortza Paris & 39,086 & 4.6 \\
Rifondazione & 35,142 & 4.1 \\
Progetto nazionalitario Sardi uniti (UDS) & 33,302 & 3.9 \\
PSd'Az & 32,859 & 3.8 \\
Socialisti democratici Italiani-Socialisti uniti (SDI-SU) & 32,245 & 3.8 \\
Alleanza popolare UDEUR & 22,610 & 2.6 \\
Comunisti Italiani & 16,010 & 1.9 \\
Indipendentzia Repubrica de Sardigna (IRS) & 9,724 & 1.1 \\
Nuovo PSI & 8,965 & 1.0 \\
Lista Di Pietro-Occhetto & 8,558 & 1.0 \\
Federazione dei verdi & 7,048 & 0.8 \\
Sardigna natzione & 5,031 & 0.6 \\
Il movimento & 3.153 & 0.4 \\
NAT & 901 & 0.1 \\
Total valid votes & 857,303 & 100.0 \\
\hline
\end{tabular}

Source: Adapted from data available through the official Web site of the Sardinian regional council: http//www.sitos.regione.sardegna.it/Elezioni-2004/index_2004.html. 
TABLE B10. Sardinian Regional Elections of 12 and 13 June 2004, Votes Cast for Regioanl Lists

\begin{tabular}{lr}
\hline Electorate & $1,449,052$ \\
Voters & $1,031,869$ \\
$\%$ Voting (of electorate) & 71.2 \\
Valid votes & 972,781 \\
$\%$ Valid votes (of voters) & 94.3 \\
Non-valid votes & 59,088 \\
$\%$ Non-valid votes (of voters) & 5.7 \\
\hline
\end{tabular}

Source: Adapted from data available through the official Web site of the Sardinian regional council: http//www.sitos.regione.sardegna.it/Elezioni-2004/index_2004.html.

TABLE B11. Sardinian Regional Elections of 12 and 13 June 2004, Valid Votes for Regional Lists

\begin{tabular}{lccl}
\hline Regional list & Valid votes & \% Valid votes & Candidate \\
\hline Sardegna insieme $^{a}$ & 487,692 & 50.1 & Soru Renato \\
Sardegna unita $^{b}$ & 394,271 & 40.5 & Pili Mauro \\
Sardigna libera $^{c}$ & 36,720 & 3.8 & Sanna Giacomo \\
UDS-NAT-Il movimento $^{\text {IRS }}$ & 35,460 & 3.6 & Floris Mario \\
Total valid votes & 18,638 & 1.9 & Sale Gavino \\
\hline
\end{tabular}

${ }^{a}$ The coalition Sardegna insieme includes DS, La Margherita, Progetto Sardegna, RC, SDI-SU, AP UDEUR, CI, Lista Di Pietro-Occhetto, and Fed. verdi.

${ }^{b}$ The coalition Sardegna unita includes FI, UDC, AN, Riformatori, Fortza Paris, and Nuovo PSI.

'The coalition Sardigna libera includes PSd'Az and Sardigna natzione.

Source: Adapted from data available through the official Web site of the Sardinian regional council: http//www.sitos.regione.sardegna.it/Elezioni-2004/index_2004.html. 
Regional Consultative Referendum, 2004

TABLE B12. Consultative Referendum Concerning the Institution of the Province of Alto Friuli

\begin{tabular}{lr}
\hline Electorate & 75,283 \\
\% Electorate that is female & 51.0 \\
Voters & 38,542 \\
\% Voters who are female & 49.8 \\
\% Voters (of electorate) & 51.2 \\
Valid votes & 38,208 \\
\% Valid votes (of voters) & 99.1 \\
Non-valid votes & 334 \\
\% Blank ballots (of non-valid votes) & 35.6 \\
\% disqualified votes (of non-valid votes) & 64.4 \\
Votes in favor & 17,597 \\
Votes against & 20,611 \\
\% Votes in favor & 46.1 \\
\% Votes against & 53.9 \\
\hline
\end{tabular}

Note: On 21 March 2004, the electorate of the 43 municipalities of Alto Friuli (the mountain districts of Carnia, and of Gemonese, Canal del Ferro, and Val Canale) was invited to participate in the consultative referendum on the institution of the province of Alto Friuli. The question put to voters was: "Are you in favor of the institution of the province of Alto Friuli, to coincide with the boundaries of the mountain district of Carnia-which includes the municipalities of Amaro, Ampezzo, Arta Terme, Cavazzo Carnico, Cercivento, Comeglians, Enemonzo, Forni Avoltri, Forni di Sopra, Forni di Sotto, Lauco, Ligosullo, Ovaro, Paluzza, Paularo, Prato Carnico, Preone, Ravascletto, Raveo, Rigolato, Sauris, Socchieve, Sutrio, Tolmezzo, Treppo Carnico, Verzegnis, Villa Santina, and Zuglio-and of the mountain district of Gemonese, Canal del Ferro, and Val Canale, comprising the municipalities of Artegna, Bordano, Chiusaforte, Dogna, Forgaria nel Friuli, Gemona del Friuli, Malborghetto Valbruna, Moggio Udinese, Montenars, Pontebba, Resia, Resiutta, Tarvisio, Trasaghis, and Venzone?"

Source: Adapted from data supplied by the electoral office of the Friuli-Venezia Giulia regional council. 


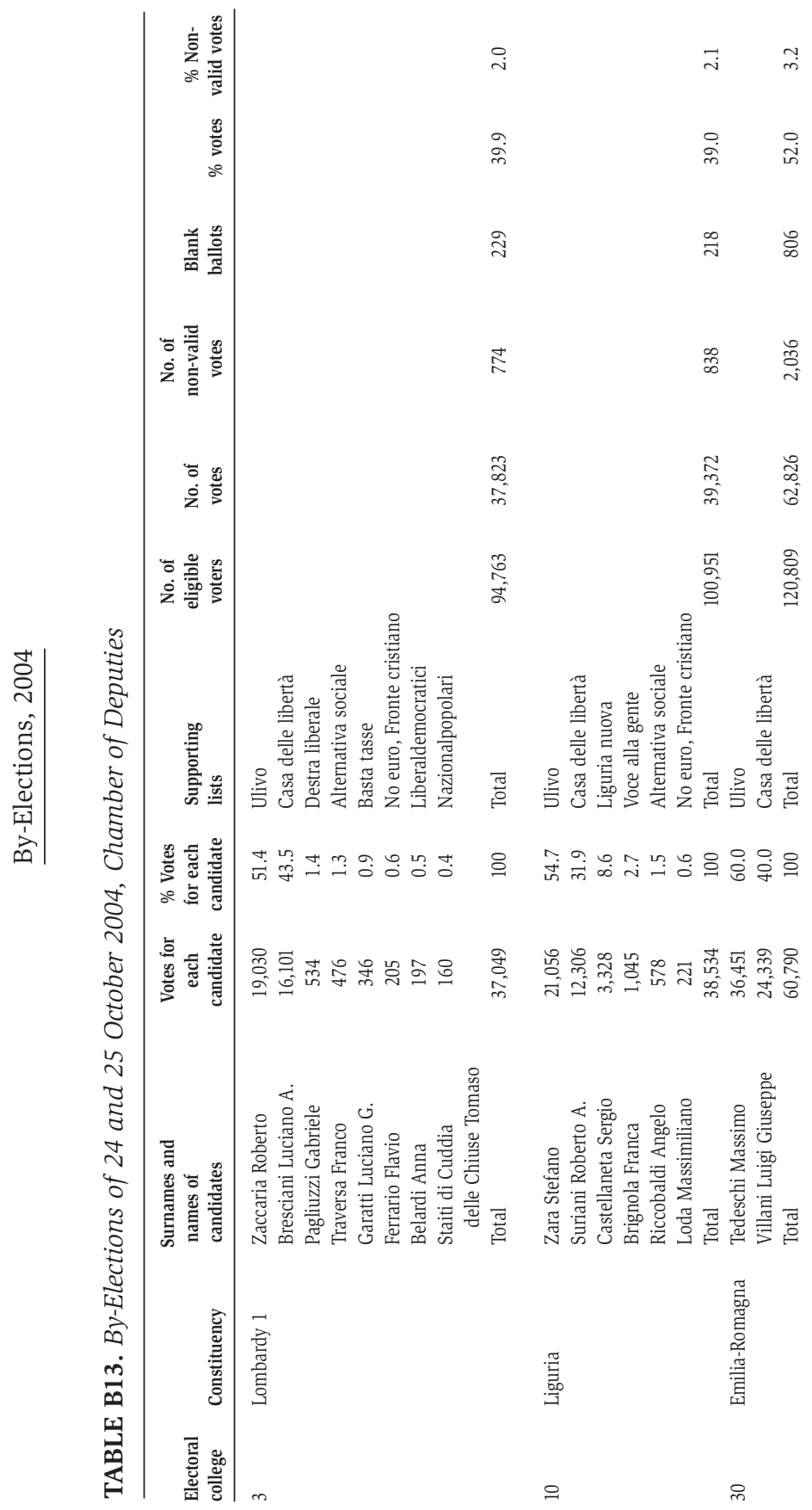




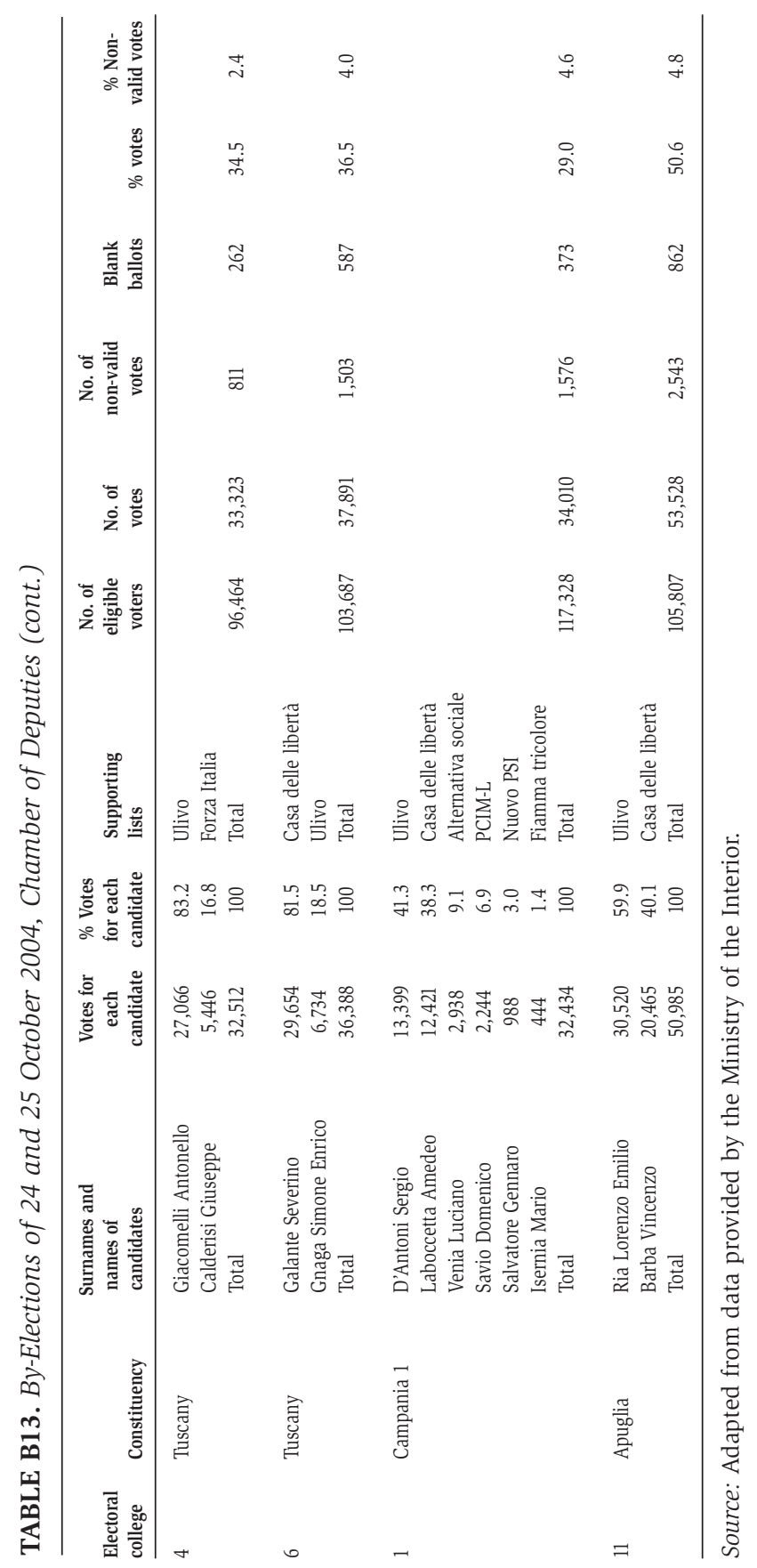




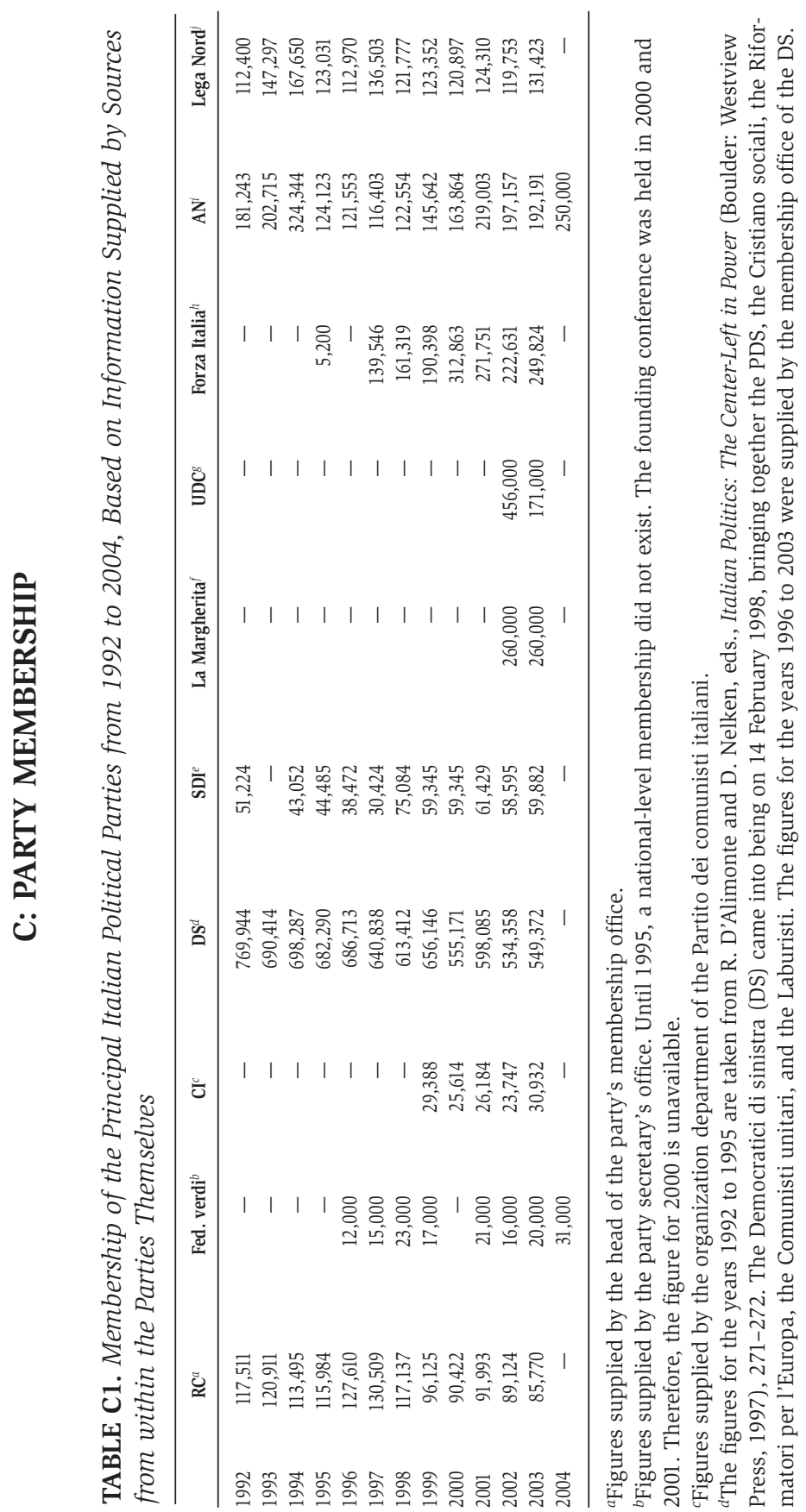




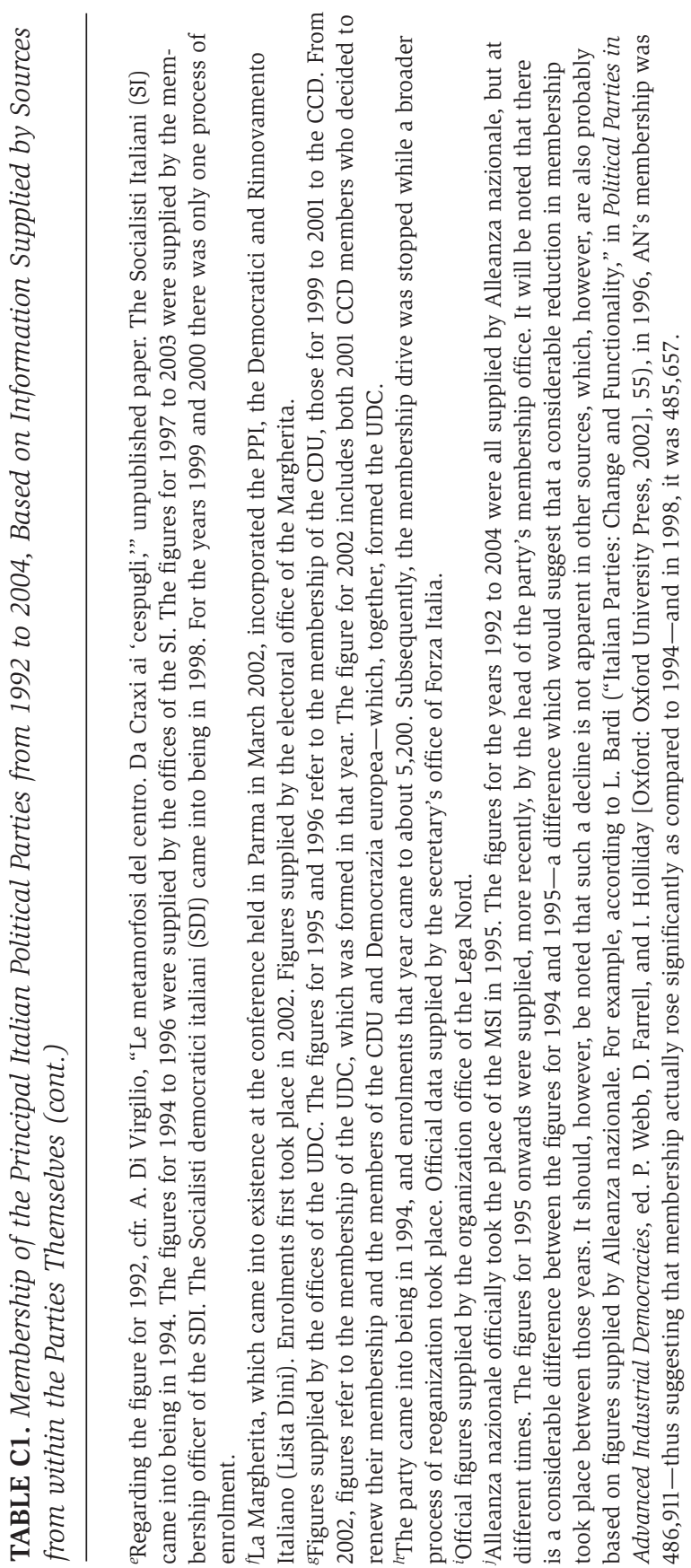

